Portland State University

PDXScholar

Fall 12-3-2013

\title{
Work Stress Reactivity and Health Outcomes: A Study of Nurses
}

Laurie Marie Jacobs

Portland State University

Follow this and additional works at: https://pdxscholar.library.pdx.edu/open_access_etds

Part of the Health Psychology Commons, Nursing Commons, and the Occupational Health and Industrial Hygiene Commons

Let us know how access to this document benefits you.

\section{Recommended Citation}

Jacobs, Laurie Marie, "Work Stress Reactivity and Health Outcomes: A Study of Nurses" (2013).

Dissertations and Theses. Paper 1515.

https://doi.org/10.15760/etd.1505

This Dissertation is brought to you for free and open access. It has been accepted for inclusion in Dissertations and Theses by an authorized administrator of PDXScholar. Please contact us if we can make this document more accessible: pdxscholar@pdx.edu. 
Work Stress Reactivity and Health Outcomes: A Study of Nurses

by

Laurie Marie Jacobs

A dissertation submitted in partial fulfillment of the requirements for the degree of

Doctor of Philosophy

in

Applied Psychology

\author{
Dissertation Committee: \\ Cynthia Mohr, Chair \\ Robert Sinclair \\ Leslie Hammer \\ Donald Truxillo \\ Matthew Carlson
}

Portland State University

2013 
(C) 2013 Laurie Marie Jacobs 


\begin{abstract}
Negative events encountered in daily life influence individual well-being. Individuals vary in their reactivity to these events, the extent to which they are behaviorally, physiologically, and psychologically influenced by them (Almeida, 2005; Neupert, Almeida, \& Charles, 2007). Reactivity to events in the form of changes in health behavior could represent either an attempt at coping (Cooper, Frone, Russell, \& Mudar, 1995) or a stressor-related failure of self-control (Muraven \& Baumeister, 2000). Such changes in behavior could have later effects on health.

Although a great deal of attention has been paid to both the immediate and longterm effects of stressors on individuals, little is understood about the potential relationship between these immediate and long-term consequences. Exploration of this connection could not only expand the understanding of the relationships between stressors, behavior, and well-being, but also inform intervention strategies.

One important domain in which stressors occur is work; certain occupations such as nursing expose individuals to a greater likelihood of experiencing stressors simply by nature of the tasks and/or environment involved. As a nursing shortage continues, stress is in fact one of the most-often cited reasons for nurses to leave the profession (Cangelosi, Markham, \& Bounds, 1998). Using a sample drawn from the Oregon Nurse Retention Project and the relatively novel statistical method of slopes-as-predictors, I examined the relationships between work stressors and nurses' health behaviors (alcohol consumption, diet, exercise) and then used those relationships as predictors of follow-up outcomes (depression, life satisfaction, perceived health).
\end{abstract}


Significant variability was found for five combinations of stressors and health behaviors, indicating that varying patterns of health behavior reactivity were indeed present in this sample; moreover four of those five stressor-behavior (reactivity) slopes emerged as significant predictors of later health and well-being. Notably, reactivity in the form of increased days of exercise during weeks of greater demands or negative events was associated with lower depression scores, and reactivity in the form of increased days of exercise during weeks of greater conflicts was associated with greater satisfaction with life. Implications of these findings, including the importance of exercise in maintaining well-being, are discussed. 


\section{Dedication}

This dissertation is dedicated to my family, especially my extremely patient soonto-be husband Damien, whose love and support made this work possible, and my parents. It is also dedicated to the members of my family who are no longer here to read this but who believed in and encouraged me, among them my grandparents, each of whom I feel tremendously lucky to have known. I would also like to thank Annie and all the other friends who supported me during this project. Each of them has made contributions to my work and to my life. 


\section{Acknowledgements}

I would like to thank my committee members, Drs. Cynthia Mohr, Robert Sinclair, Leslie Hammer, Donald Truxillo, and Matthew Carlson, for their service on my committee and for their input on and help with this dissertation. Their excellent feedback was extremely helpful in my process, and I am sincerely grateful that each of them shared their knowledge and expertise with me. Dr. Mohr, in particular, as my committee chair, advisor, teacher, and mentor, has made a tremendous contribution to this work and to my development as a scholar, for which I will always be grateful.

The Oregon Nurse Retention Project, which is the source of the data used in this dissertation, was funded by a grant from the Northwest Health Foundation (Proposal 14180). I am grateful for the support of the other members of the ONRP team, led by Drs. Sinclair and Mohr, our partners at the Oregon Nurses' Association, particularly Dr. Sue Davidson, and the nurses who participated in the study.

My student colleagues on Dr. Mohr's Daily Research Lab team were a valuable resource as we explored the slopes-as-predictors method and diary methodology together, and I would also like to express my appreciation to them. 


\section{Table of Contents}

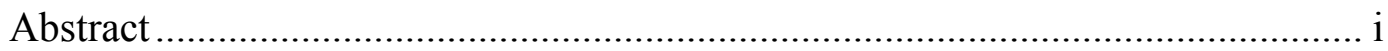

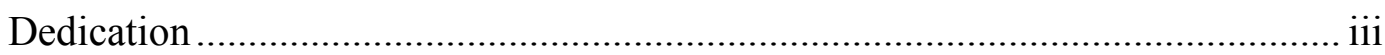

Acknowledgements ............................................................................... iv

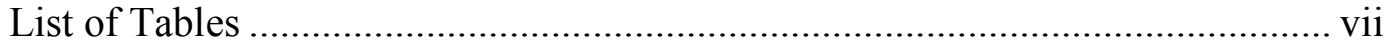

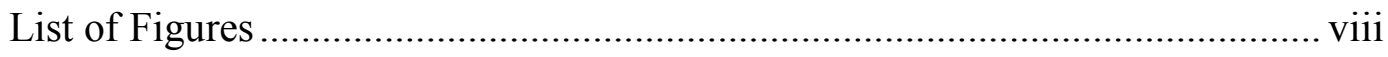

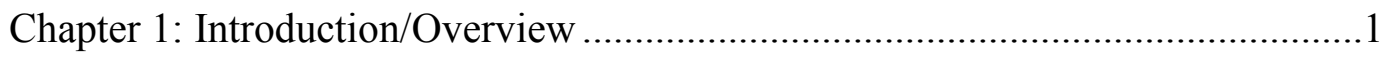

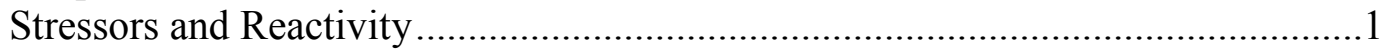

Drawing Relationships Between Reactivity and Outcomes ...............................4

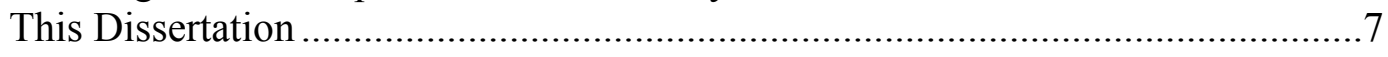

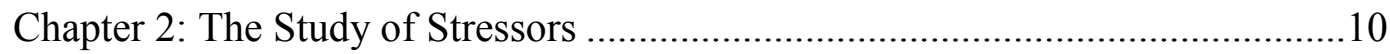

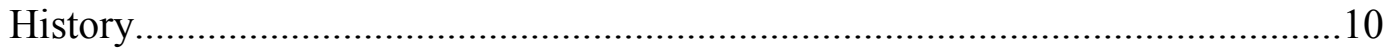

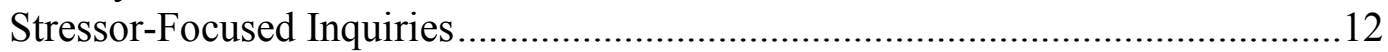

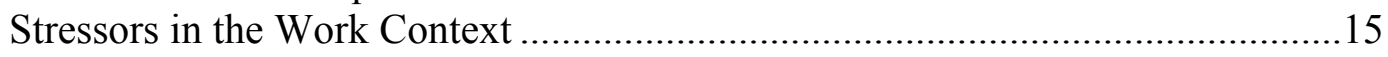

The Transactional Model of Stress and Coping................................................20

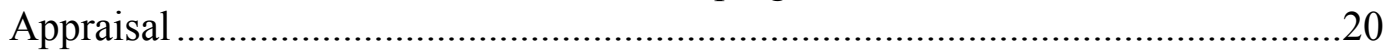

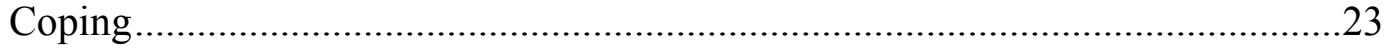

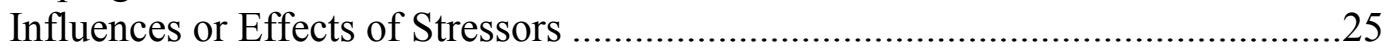

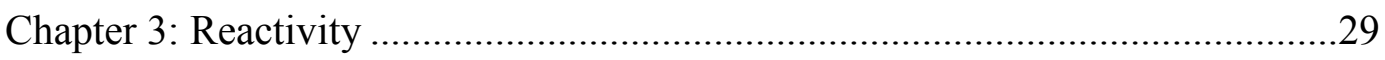

Reactivity and its Individual-Level Correlates ................................................ 31

Situational/Dynamic Influences on Reactivity .................................................34

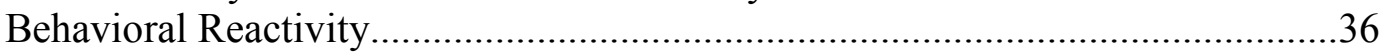

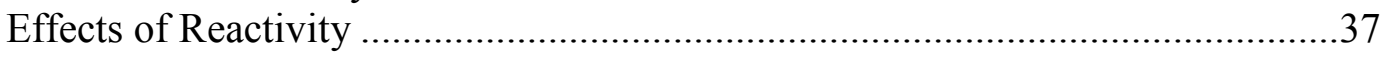

Chapter 4: Behavioral Reactivity, Health, and Well-Being...............................40

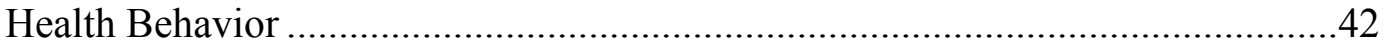

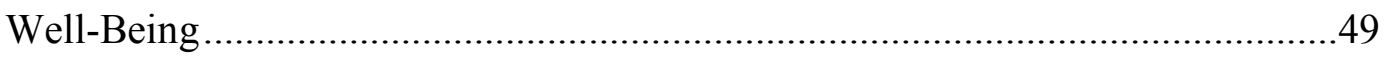

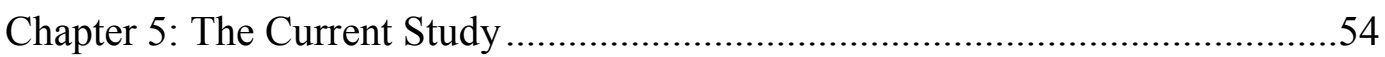

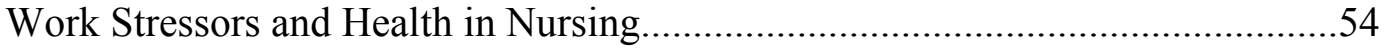

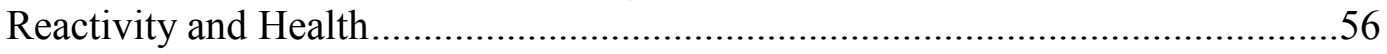

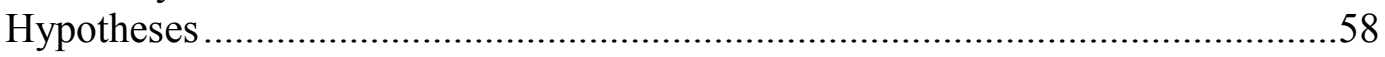

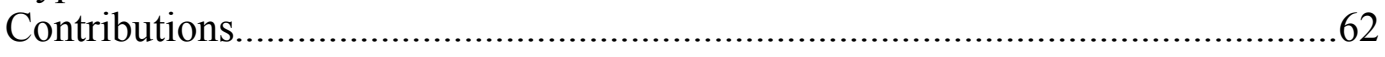

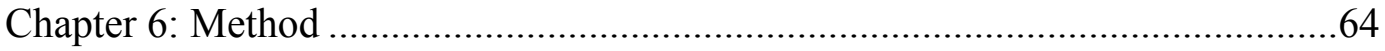

The Oregon Nurse Retention Project..........................................................64

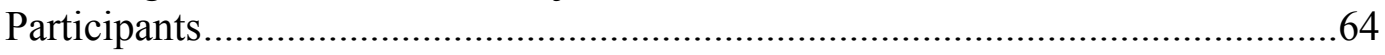

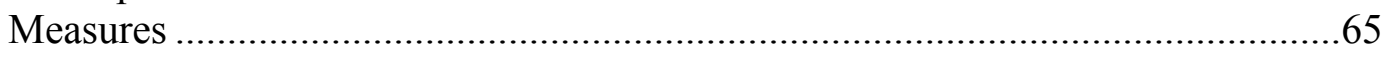

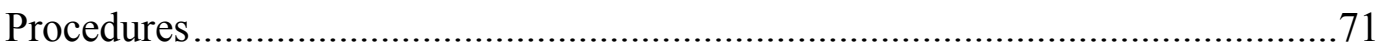

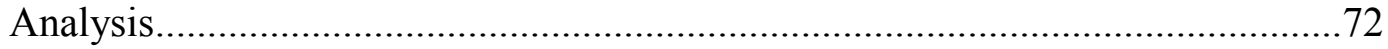




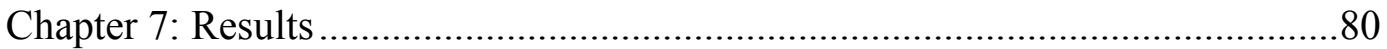

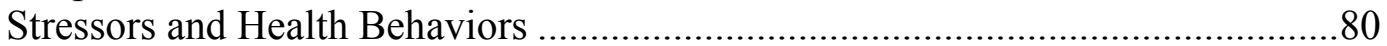

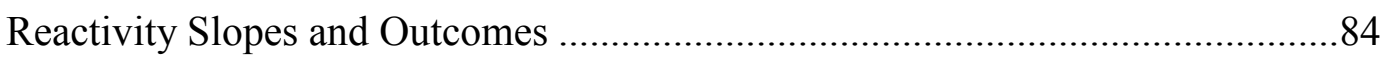

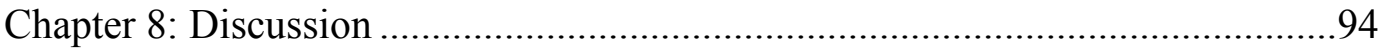

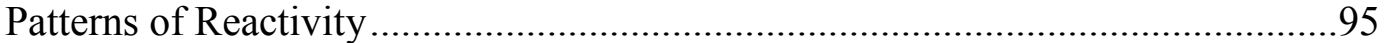

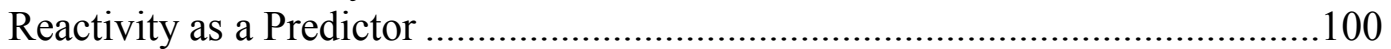

Limitations and Suggestions for Future Studies ..........................................106

Contributions, Implications, and Conclusion..............................................110

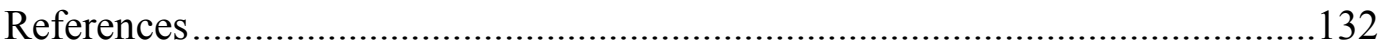

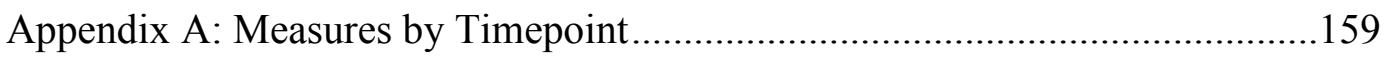




\section{List of Tables}

Table 1. Hypothesized Reactivity-Outcome Relationships .............................115

Table 2. Correlations of Slope Components ...................................................116

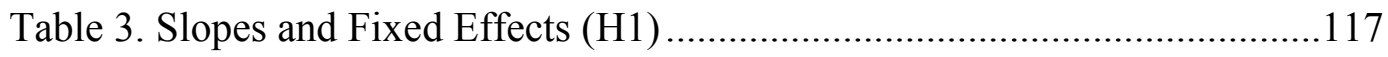

Table 4. Slope, Outcome, and Covariate Descriptive Statistics ........................118

Table 5. Correlations of Slopes and Outcomes...............................................119

Table 6. Regressions of CES-D Scores on Slopes (H2a) ...............................120

Table 7. Regressions of SWLS Scores on Slopes (H2b) .................................121

Table 8. Regressions of Perceived Health on Slopes (H2c) .............................122 


\section{List of Figures}

viii

Figure 1. The Transactional Model of Stress and Coping ...............................123

Figure 2. Immediate and Longer-Term Reactions to Stressors ..........................124

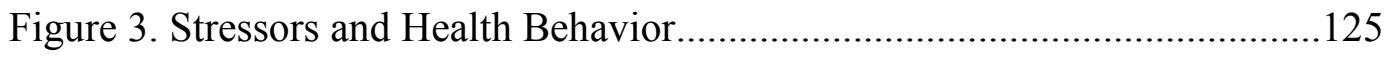

Figure 4. The Oregon Nurse Retention Model ..............................................126

Figure 5a. Mean Weekly Conflicts-Exercise Days Slopes ...............................127

Figure 5b. Mean Weekly Demands-Exercise Days Slopes ..............................128

Figure 5c. Mean Weekly Negative Events-Exercise Days Slopes .....................129

Figure 5d. Mean Weekly Negative Events-Negative Minutes Slopes.................130

Figure 5e. Mean Weekly Conflicts-Drinking Days Slopes ..............................131 
Chapter 1: Introduction/Overview

\section{Stressors and Reactivity}

Events of individuals' everyday lives can influence them in a number of ways. Negative or stressful events may have an immediate effect, in the form of emotional reactions (Bolger, DeLongis, Kessler, \& Schilling, 1989; David, Green, Martin, \& Suls, 1997), which may in turn influence behavior (Macht \& Simons, 2000; Mohr, Brannan, Mohr, Armeli, \& Tennen, 2008). Longer-term, stressful events can contribute to the development of mood disorders (Hartley, Violanti, Fekedulegn, Andrew, \& Burchfiel, 2007; Rabkin, 1993) and health problems (McEwen, 1998a) on an individual level; on a societal level, they may contribute to worker shortages in occupations such as nursing, where nurses often report stress to be a major contributing factor to their decision to change careers (Cangelosi et al., 1998). Events do not have to be large or traumatic to affect individuals; minor negative life events, or stressors, can in fact be more predictive of distress than major ones such as divorce or bereavement (Pillow, Zautra, \& Sandler, 1996). Researchers continue to investigate how and why individual reactions to events vary; however immediate reactions to events are usually studied separately from their longer-term consequences, and the connections between them are still largely unexplored.

One important factor at work in individuals' immediate reactions to their experiences is reactivity, the extent to which events, negative or positive, influence them psychologically and physiologically (Almeida, 2005; Neupert et al., 2007). Reactivity is often viewed as a relatively stable individual difference (S. Cohen \& Hamrick, 2003), and has been linked to certain patterns of personality traits, particularly neuroticism (Bolger \& Zuckerman, 1995; Suls \& Martin, 2005). However, reactivity has also been found to 
vary with age (Mroczek \& Almeida, 2004; Neupert et al., 2007; Sliwinski, Almeida, Smyth, \& Stawski, 2009) and may be altered by life experiences (Lepore, Miles, \& Levy, 1997), suggesting that it might be a more dynamic construct rather than a wholly stable individual difference. Reactivity can also be influenced by contextual factors such as perceived control (Neupert et al., 2007), global stress levels (Sliwinski et al., 2009), and depression (Steger \& Kashdan, 2009), further supporting this proposition. Thus, an individual's level of reaction to a given event may be influenced by both her individual characteristics or life circumstances and the features and context of the event itself.

Reactivity can be measured in multiple ways. Physiological reactivity is most often measured in the lab or under other controlled settings (Schwebel \& Suls, 1999), whereas psychological reactivity is often measured through emotional and behavioral responses to naturalistically encountered stressors (L. H. Cohen, Gunthert, Butler, O’Neill, \& Tolpin, 2005; Lemke, Brennan, Schutte, \& Moos, 2007), typically through experience sampling diary methods. In such a design, participants might be asked to report their experiences of events, moods, or behaviors at regularly scheduled intervals. The fact that individuals vary in the events that they experience, their perceptions of them, and their level of reactivity to them indicates that within-person analyses of longitudinal data are necessary to fully explore these patterns. In studies employing within-person techniques, physiological reactivity to stressors has been found to be a vulnerability factor for hypertension (Ming et al., 2004), and psychological reactivity to stressors has been found to be a vulnerability factor for physical health problems and affective disorders such as depression (Charles, Piazza, Mogle, Sliwinski, \& Almeida, 2013; Parrish, Cohen, \& Laurenceau, 2011; Piazza, Charles, Sliwinski, Mogle, \& 
Almeida, 2012). This suggests that both major ways of measuring reactivity may relate to negative health and well-being outcomes. Because I chose to examine reactivity to the naturalistically encountered stressors of nursing work, this dissertation is focused on the effects of psychological reactivity.

Effects of stressors can be seen through global measures of well-being such as depression symptoms or subjective well-being in the longer-term (Gomez, Krings, Bangerter, \& Grob, 2009; Hartley et al., 2007), or through measures of psychological reactivity (e.g., more immediate changes in affect or behavior). Immediate consequences of stressors for individuals may include experiencing heightened negative affect or negative perceptions of their situation (Bolger et al., 1989; Koerner \& Kenyon, 2007), or engaging in tension-reduction drinking (Conger, 1956) or other potentially negative health behaviors such as poor eating habits (Newman, O’Connor, \& Conner, 2007). In fact, evidence suggests that the volitional health behaviors (i.e., drinking, smoking, diet, exercise) that many people find challenging to self-regulate under normal circumstances may become even more difficult for them to exercise control over when they have also been coping with stressors (Muraven \& Baumeister, 2000). Therefore stressor-related changes in health behavior could be one pathway through which stressors may impact individuals' later health.

These health effects might be particularly pronounced in those who are engaged in certain types of work, given that some occupations expose individuals to a greater likelihood of experiencing stressors simply by nature of the tasks and/or environment involved. One such occupation is nursing, where both responsibility for the health of others and exposure to individuals (e.g., other medical personnel, patients, patients' 
families) who may themselves be facing challenging or emotionally intense situations provide numerous opportunities for nurses to experience stressors (Chang, Hancock, Johnson, Daly, \& Jackson, 2005; Lambert, Lambert, \& Yamase, 2003). The physical health of nurses has been well-researched, particularly by the Nurses' Health Study (NHS; Colditz, Manson, \& Hankinson, 1997), a multi-decade longitudinal study that has provided evidence of linkages between various physical and behavioral risk factors and disease, including harmful effects of alcohol consumption and poor eating habits, both of which are associated with increased cancer risk. In addition to their potential negative effects on nurses' physical and mental health (Bradley \& Cartwright, 2002; Greenglass, Burke, \& Moore, 2003), stressors may also contribute to the nursing shortage (Cangelosi et al., 1998). Thus role stress, particularly work overload, is one of the primary areas of concern in the nursing literature (Chang et al., 2005); however McNeely (2005) suggests that the linkages between work stressors and nurse health have not been thoroughly explored.

\section{Drawing Relationships Between Reactivity and Outcomes}

A great deal of attention has been paid to both the immediate and long-term effects of stressors on individuals, but little is understood about the potential relationship between these immediate and long-term consequences. Some studies have focused on measures of physiological reactivity and linked it to later outcomes (e.g., Ming et al., 2004). Longitudinal research in this area is relatively uncommon, however, as is research on psychological rather than physiological reactivity. Thus few studies have measured psychological reactivity to naturally encountered stressors and explored its relationship to later outcomes. The small number of existing studies of this type of which I am aware 
have focused primarily on the etiology and treatment of depression (e.g., L. H. Cohen et al., 2008, 2005; Parrish et al., 2011) and on linking affective reactivity with later physical and mental health problems (e.g., Charles et al., 2013; Piazza et al., 2012). Recently, however, Dr. Cynthia Mohr and her research team, including myself, have begun to explore how daily relationships between mood and alcohol consumption link to later outcomes in a community sample (Mohr et al., 2013). This new approach of connecting behavioral reactivity with outcomes may have implications for intervention.

One question often pursued in stress research is when and how to intervene in the process to help prevent individuals from experiencing negative stress-related outcomes. Given that many stressors cannot be prevented, a greater understanding of the ways in which reactivity to stressors relates to longer-term outcomes would help to shed light on that question. For instance, in the case of the Parrish et al. (2011) study of emotional reactivity and symptoms of depression, in which they found that greater immediate reactivity to negative events is linked to higher levels of depression symptoms later, this understanding might suggest pathways for intervention, such as using measures of reactivity to identify clients who are at risk for an increase in symptoms, or developing coping strategies clients can use to dampen reactivity when negative events occur.

Further exploration of the connections between reactivity and longer-term consequences of stressors, therefore, could determine whether or not reactivity is predictive of outcomes such as depression above and beyond its relationship to previously known predictors of those outcomes such as age and neuroticism. This could not only expand the understanding of the relationships between stressors, behavior, and well-being, but also inform intervention strategies. Novel statistical methods, specifically 
the slopes-as-predictors method, in which short-term individual-level (within-person) relationships between variables, such as stressors and behavior, can be used as predictors of later outcomes, make this kind of analysis possible (L. H. Cohen et al., 2005; Mohr et al., 2013).

Individual emotional and behavioral reactions to stressors can take a variety of forms, such as changes in an individual's use of alcohol. Some individuals might drink more than usual when they encounter more stressors, perhaps as an attempt to cope via the phenomenon of tension-reduction drinking (Conger, 1956). Others might drink less during weeks when their number of stressors is higher than usual, but more during weeks when that number is lower than usual, exhibiting perhaps instead a pattern of enhancement drinking; drinking to increase enjoyment during good times (Cooper et al., 1995), or perhaps simply finding themselves too busy during higher-stressor weeks to drink. By gathering multiple longitudinal measurements of stressors and a variety of potential reactions, it is possible to describe the relationship between them, for instance whether a given individual tends to drink more, less, or the same amount of alcohol as usual during weeks in which they encounter a greater number of stressors than is usual for them. In many studies, these patterns themselves, which can be quantified as individual slopes, would be the outcomes of interest. However immediate reactions such as these may also prove to be potentially powerful predictors of later outcomes (L. H. Cohen et al., 2005; Mohr et al., 2013). Drinking to cope, for instance, is believed to be linked to negative consequences such as alcohol problems (Cooper et al., 1995). Using these relationships as predictors offers a new way in which to test that proposition by first 
empirically describing the immediate relationship between stressors and drinking and then seeing how patterns in those relationships link to later outcomes.

\section{This Dissertation}

In this dissertation, I used data on stressors, health behavior, and outcomes from a longitudinal sample of nurses to examine how psychological reactivity to work stressors relates to longer-term well-being. Work stressors are of particular interest and usefulness because of their potential role as contributors to the nursing shortage and because of the many opportunities to encounter such stressors in everyday nursing work. I explored these patterns using data collected as part of the Oregon Nurse Retention Project (ONRP, Sinclair et al., 2009), a larger study focused on nurse retention and turnover. I was a member of the ONRP team. As part of that effort, we collected data from nurses that spanned multiple domains that could potentially influence nurses' decision to remain in or leave their job and their profession, including work events and contextual variables. The ONRP data collection included weekly diaries from nurse participants containing measures of work stressors and health behaviors, as well as follow-up measures of wellbeing. Whereas the ONRP as a whole was focused on job retention and turnover as outcomes, my dissertation is focused specifically on the relationship between reactivity and health and well-being outcomes. Using this sample, I examined the relationships between nurses' work stressors and reactivity to those stressors as demonstrated by immediate changes in health behaviors (alcohol consumption, diet, exercise), and then used those relationships as predictors of follow-up outcomes (depression, life satisfaction, perceived health). 
Contributions. The linkage between psychological reactivity and longer-term outcomes is still largely unexplored, with existing studies focused primarily on depression. Those existing studies, therefore, have also focused primarily on clinical samples, so little is known about how reactivity might link to outcomes in a community sample. Through the use of the slopes-as-predictors technique to examine longitudinal data from a sample of nurses, this dissertation offers the first in-depth examination of the relationships between psychological reactivity to work stressors and later health and wellbeing outcomes. This dissertation thus also has the potential to contribute to the understanding of factors relating to the well-being of nurses, who are a population of concern for many researchers and policymakers given the nursing shortage and growing demand in the field (Cangelosi et al., 1998). If links between reactivity and later wellbeing such as those found here continue to hold up empirically, nurses and their employers could be educated about these patterns of stressors and behavior and then use that awareness to interrupt those patterns and thereby perhaps prevent later negative consequences.

Finally, research linking stressors to later health and well-being has focused primarily on the mere occurrence of stressors as the predictor, which allows for the discovery of empirical evidence of a link between events and outcomes, but not the ways in which events lead to outcomes. Direct physiological effects of stressors such as allostatic load are relatively well-understood (McEwen, 1998b), but other potential pathways from stressors to outcomes remain unclear. The relationship explored here between reactivity, as measured through stressor-related changes in health behavior, and later health and well-being outcomes has begun to explicate one of the pathways by 
which stressors may link to outcomes. 


\section{Chapter 2: The Study of Stressors}

\section{History}

Research interest in stress, and by extension stressors, came about primarily in the mid-to-late $20^{\text {th }}$ century (S. Cohen, Kessler, \& Gordon, 1997; Lazarus \& Folkman, 1984). Stress research is a large field, encompassing areas of study that overlap across multiple social and medical science domains. With numerous disciplines converging in one broad area of study, it is perhaps not surprising that definitional problems arose.

Definitions of "stress" vary widely, to the point where one review article begins by talking about the fact that review articles always seem to begin with a discussion of the definitional issue (Vingerhoets \& Marcelissen, 1988). Perhaps the most commonly used set of definitions in the social sciences is drawn from Hooke's $17^{\text {th }}$ century engineering framework, in which "load" is the force on an object, "stress" is the ratio of the pressure to the object's size, and "strain" is the resulting change, such as a shelf bowing under too much weight (Lazarus, 1993a). Thus in a human context, stress might refer to the demands placed on an individual (often relative to their resources), while strain would refer to the effects of stress on that person. It is important to note that these demands may not be inherently harmful. Stressors, therefore, are "external events or conditions that affect the organism" (Breznitz \& Goldberger, 1993, p. 3). Use of this terminology is far from universal. Hans Selye, one of the earliest researchers on the physiological effects of stress, used the term stress to describe the result of demands on an organism; in other words, he referred to strain as stress (Selye, 1993). Jex and Beehr (1991), looking at the topic from the perspective of organizational and occupational health psychology, recommended "stress" refer to the entire area of study, "stressor" for 
job conditions that employees must respond to (such as constraints or overload), "outcome" for reactions to these stressors, and "strain" for outcomes that are negative. In this dissertation, I will use the stress-strain-stressor terminology, except in cases where the literature being discussed does not.

Current research into stress and stressors has its roots in multiple pathways. Selye's work on the effects of physiological stressors, such as disease or injecting an animal with hormones, began in the 1930s using animal models to explore the possibility that there was a standard physiological response (the General Adaptation Syndrome) that could be provoked by numerous disparate situations and stimuli (Selye, 1993). Contemporary to Selye's early work, Adolf Meyer, a medical doctor operating from a practice standpoint, began to explore the possibility that major events of individuals' lives such as bereavement might impact their health (S. Cohen et al., 1997; Dohrenwend, Raphael, Schwartz, Stueve, \& Skodol, 1993). On the psychological side, there was growing recognition that the symptoms displayed by some war veterans, such as the syndrome referred to as "shellshock" following World War I, might be a result of their traumatic experiences in combat, and thus after World War II a strong interest in understanding the psychological effects of trauma arose (Lazarus \& Folkman, 1984; Lazarus, 1999).

However, psychologists quickly began to struggle with what is perhaps one of the largest questions in the field -individual differences in response to apparently similar events. Individuals who experience what seem to be the same major life events or traumas often do not show the same reactions to them, and one may become distressed while another does not. For instance, not all World War I and II combat veterans 
displayed the symptoms that led to researchers' concern, even those who served side-byside. As research on stressful experiences and the distress they sometimes cause continued to develop, it became clear that there was more at work in determining individuals' reactions than just exposure to traumatic events, or even to normative major life events such as bereavement (Lazarus, 1993b; Lazarus \& Folkman, 1984). Attempts to understand the wide range of individual variability in reactions to events developed into two broad paths of inquiry — one focused on the context and characteristics of the stressor, the other focused on the individual. Individual-focused inquiries have focused primarily on factors that might lead individuals to be more or less likely to encounter and attend to various types of stressors, e.g., personality (Bolger \& Zuckerman, 1995) and demographics (Almeida \& Horn, 2004). Although they may be useful for examining the validity and explanatory power of my reactivity measures in the analysis phase, these individual characteristics are not a primary focus of this dissertation, therefore for the purposes of this literature review I will offer a more in-depth examination of the research focused on stressors.

\section{Stressor-Focused Inquiries}

The expansion of research on stressors over time has included both an increase in the types of events being considered and additional examination of their features. Combat was clearly not the only situation in which individuals might be exposed to traumatic events, so that area of research ultimately expanded to include other traumas such as violent victimization, childhood abuse, disasters, and serious motor vehicle accidents (Hartley et al., 2007; Pennebaker, 1989; Pynoos, Sorenson, \& Steinberg, 1993). Research into life events (which are distinct from traumas in that the events considered 
are typically more normative) continued to focus on the occurrence of major change events (e.g., bereavement) until the late 1960s, when Holmes and Rahe expanded on existing checklist measures of such events by developing the Social Readjustment Rating Scale. This new measurement tool incorporated ratings of how much adjustment each event might be expected to require, as well as including more common events that may require adjustment such as Christmas or vacations (Holmes \& Rahe, 1967; Turner \& Wheaton, 1997).

Examination of traumas and major change events alone, however, was doing an inadequate job of accounting for distress, so the explosion of research on stress in the 1970 s and 80 s expanded the lens much further to include more commonly encountered stressors and daily hassles (Almeida, 2005; Bolger et al., 1989; Pillow et al., 1996; Vingerhoets \& Marcelissen, 1988). This line of research has indeed offered additional predictive and explanatory power to the field. Bolger, DeLongis, Kessler, and Schilling (1989) found that daily stress accounted for up to $20 \%$ of variance in daily mood, a number higher than that of previous studies, which they suggested might relate to their inclusion of interpersonal conflicts in the list of events. Pillow et al. (1996) found that minor stressors not only mediated the effects of major events on distress, but also accounted for more of the variance in distress than major stressors did, even though they had oversampled individuals who had experienced major life events to make sure they had a sufficient number of these less common stressors in their sample. Furthermore, Wagner, Compas, and Howell (1988) found that negative daily events fully mediated the relationship between major negative events and psychological distress. Previous studies had also found daily events to be significant predictors of physical and psychological 
symptoms (DeLongis, Coyne, Dakof, Folkman, \& Lazarus, 1982; Kanner, Coyne, Schaefer, \& Lazarus, 1981).

Because of the demonstrated explanatory power on distress and the greater prevalence in general population samples of more frequently experienced/smaller events, they are now much more commonly incorporated into stress research. However such events, and individuals' reactions to them, may be less memorable in retrospect than less common major life events like the loss of a job, making accurate retrospective recall a challenge. Therefore there has also been a narrowing of the time frame of measurement in many studies, leading researchers to gather information from participants weekly, daily, or even multiple times per day (Eckenrode \& Bolger, 1997). Use of such repeated measures and their accompanying within-person analysis techniques now allows the examination of patterns of intra-individual associations between events and reactions in addition to the more traditional inter-individual differences. One area of exploration has been the types of events individuals encounter. Vingerhoets and Marcelissen (1988), for instance, listed seven categories of stressors in addition to life events, among them several subtypes of trauma, daily hassles, and "stressors associated with the living and work situation" (p. 283).

Given the breadth of domains in which events may be encountered, some studies categorize events, or even limit their scope to include only particular categories of events, such as interpersonal interactions with close others or events taking place in the work setting (Almeida \& Horn, 2004; Almeida, Wethington, \& Kessler, 2002; Hartley et al., 2007). This allows researchers to compare reactions to similar events, such as partner conflicts, to one another rather than to events from another domain that may be likely to 
hold different meaning and significance to individuals. Similarly, some researchers in occupational health psychology (OHP), a field concerned with workers' health and wellbeing from a standpoint of both preventing work-related problems and increasing wellbeing in the workplace (Johnson, 1996; Macik-Frey, Quick, \& Nelson, 2007; J. C. Quick, 1999), have narrowed the field of study to focus only on work-related events. Such studies often take place in an occupational setting in which stressors (and thus also the negative consequences of stressors) are relatively common. Although many occupations may fit that definition, researchers often choose to focus on occupations that also serve key societal functions such as law enforcement, air traffic control, medicine, or the military (e.g., Stephens \& Pugmire, 2008; O’Connor, O’Connor, White, \& Bundred, 2000; Repetti, 1993); understanding and preventing negative stressor-related consequences for individuals in such occupations can directly benefit the health of society as well as that of the employees themselves.

\section{Stressors in the Work Context}

As a context in which individuals tend to spend a great deal of time and energy, work offers numerous opportunities for stressors and positive events to take place. In one study of daily events with a treatment sample, a work-related event was rated as the worst stressor of the day more than a quarter of the time (Gunthert, Cohen, Butler, \& Beck, 2005). A great deal of OHP and occupational health research, in fact, has concentrated on stress, including psychosocial stressors such as control, role stress, and job demands (Danna \& Griffin, 1999; Ganster \& Schaubroeck, 1991; Jex \& Beehr, 1991). As in many areas of research, many of the studies examining the relationships between work stressors and strains have been cross-sectional; Zapf, Dormann, and Frese (1996) conducted a 
methodological review of the longitudinal studies available at that time and found that the studies that most closely matched their recommendations for eliminating reverse causation and third variable explanations did indeed find longitudinal links between work stressors and strains.

Work stress and strain, especially chronic stress or strain, have been linked with numerous physical health problems, including metabolic syndrome, high cholesterol, elevated blood pressure, and cardiovascular disease (Chandola, Brunner, \& Marmot, 2006; O’Connor et al., 2000; Rose et al., 2006; Schnall, Landsbergis, \& Baker, 1994). One meta-analysis of longitudinal studies in fact suggested that those who experience work stress have a 50\% greater risk of coronary heart disease (Kivimäki et al., 2006). In addition to physical health outcomes, mental health outcomes such as depression and poorer psychological well-being have also been associated with work stressors (Kopp, Stauder, Purebl, Janszky, \& Skrabski, 2008; Stephens \& Pugmire, 2008). In a longitudinal study by Spector, Chen, and O'Connell (2000), job stressors remained associated with job strains even when levels of prior strains and negative affectivity were taken into account.

One job where stressors may be quite common and have multiple impacts is nursing, where stress and strain on nurses have the potential to not only affect individuals' well-being, but also that of the patients nurses care for. Nursing also offers the potential for rewarding experiences such as helping patients' health improve. Nurses have been the focus of many studies, both because of the high potential within nursing work for exposure to stressors and because of ongoing concerns about a nursing shortage as nurses leave the profession (Cangelosi et al., 1998; Chang et al., 2005; McGrath, Reid, 
\& Boore, 1989). Work stress was in fact cited by $42 \%$ of nurses in one study as a major reason that they change jobs (Cangelosi et al., 1998).

Several aspects of nursing work and the nursing environment seem to be particular areas of focus in the empirical literature. A review of studies of nurses found that most nurse stress research has focused on factors in the work environment such as control, shift work, and work responsibilities (Lambert \& Lambert, 2001), an emphasis that has continued as role stress, particularly a perception of work overload, remains one of the primary concerns in the nursing literature (Chang et al., 2005). Nursing in hospitals is a physically demanding profession that requires 24-hour coverage, which may lead to stress and strain as nurses struggle to arrange their schedules at work and at home, particularly if they are working rotating (i.e., periodically changing from a daytime shift to swing shift, swing shift to graveyard, etc.) rather than fixed shifts (Chang et al., 2005; Jamal \& Baba, 1992). Nurses may also experience workplace violence, including physical assaults, threats, sexual harassment, and verbal abuse; one study found annual rates of 12 physically violent incidents and 38.5 non-physical violent incidents per 100 registered nurses (Gerberich et al., 2004).

Nurses' health is another area of concern. Given nurses' regular contact with patients who may have weakened immune systems, if nurses are themselves more susceptible to infectious disease because of work stress (S. Cohen \& Hamrick, 2003) they could run the risk of spreading colds or flu among their patients. Bradley and Cartwright (2002) examined nurse stress, social support, and health, and discovered that hassles, particularly those related to workload, were negatively related to nurses' health. They also found that nurses who felt their organization was supportive of them tended to be in 
better health. In addition to physical health, nurses' mental health may also be adversely affected by workload. Greenglass et al. (2003) found that workload stressors were related to anger, cynicism, and emotional exhaustion, as well as to depression.

Several models of work stress are informative as a context for the key variables of interest in this dissertation. Person-environment fit, a concept drawn from the social psychology literature, suggests that stress and strain arise from mismatches between the person and the job, for instance if an individual's skills are not a good match for the job's requirements, or a job is not meeting an individual's needs. This framework lends itself to studies that measure fit or lack thereof (Ganster \& Schaubroeck, 1991), and although reactivity as an individual difference might be considered a person-level factor, the focus on goodness of fit does not seem the best lens under which to view the topic of individual reactions to stressful situations or events, which is the focus of this dissertation.

Demands-control, or decision latitude, incorporates the idea of the demands of work, and suggests for instance that a highly demanding job where the employee also has a great deal of control will be less stressful than a highly demanding job where the employee lacks control. Although there is empirical evidence that job demands and control have an impact on psychological well-being (Häusser, Mojzisch, Niesel, \& Schulz-Hardt, 2010), in the case of this dissertation, that model does not adequately account for the individuallevel processes of interest. Although reactivity may take place in response to work demands, it is likely that many nurses have similar limits to how much control they can exert, given the structure of the hospital hierarchy.

Workload and situational constraints within a job are potential stressors, and both are players in the job demands-resources framework, in which the balance of a job's 
demands and the individual's available resources to meet those demands leads to burnout if an individual is overtaxed, or engagement if the individual is rich in resources (Schaufeli \& Bakker, 2004). Reactivity could potentially be conceptualized as taking place within this framework, but for the purposes of this dissertation, which represents an initial examination of reactivity and outcomes using the slopes-as-predictors analysis technique, I opted not to include potential protective factors such as resources in my analyses. The possible use of such factors in future research is discussed in Chapter 8 . Another potential set of work stressors is role stressors, which might exist within a job, or between work and home as individuals struggle with competing demands from each arena (J. C. Quick, 1999). Such stressors within jobs are also potential predictors of affective outcomes and therefore within the scope of the negative events examined in this dissertation (Jex \& Beehr, 1991), although non-work or work-family role stressors were not included in the ONRP. The ONRP as a whole was framed around the Oregon Nurse Retention Model (ONRM), which is discussed in more detail in Chapter 5. The primary source for the theoretical framework of this dissertation is the transactional model of stress and coping, discussed below.

Whether the domains, contexts, or types of events considered are specific or broad, current research on stressors often encompasses not only the occurrence of events, but also an individual's perception of them, leading to transactional models of stress and coping that incorporate external and internal factors (Derogatis \& Coons, 1993; Lazarus \& Folkman, 1984). As described by these models, an event that one individual perceives as stressful might go almost unnoticed by another, and even if both individuals perceive the event as stressful, their perception of the event's qualities may be different. 
Accordingly, many measures of life events and daily stressors now incorporate questions about the features of these events (such as controllability, undesirability, and threat level) in addition to asking about whether or not they occurred (Turner \& Wheaton, 1997).

\section{The Transactional Model of Stress and Coping}

A "transactional" model of stress and coping refers to the interactive relationship between person and environment. Rather than a linear and perhaps unidirectional relationship, in which the environment acts on the person, for instance, such models represent the person-environment relationship in stress and coping as a dynamic and iterative process in which characteristics of each influence the other. Reactivity can be conceptualized as part of that process, as will be described in the next chapter. The person-environment transactions described in this model tend to occur over a relatively short timespan and may vary widely from individual to individual, and are thus often studied using within-person diary methods. While many perspectives now incorporate transactional elements, the model referred to as the transactional model of stress and coping was developed by Lazarus and Folkman (1984), and lays out the process along the pathway shown in Figure 1.

\section{Appraisal}

Appraisal is a key part of the stress and coping process in most transactional models (Moos \& Schaefer, 1993). As described by Lazarus and Folkman (1984), a situation or event which might be a potential stressor is evaluated, or appraised, by the individual. This is where one determines what, exactly, is happening, and how, if at all, it is self-relevant. This may be a multi-step process, beginning with primary appraisal, wherein one determines which of three categories the event belongs to: irrelevant, 
benign-positive, or stressful. For instance, if one encounters an unexpected traffic jam while not on a schedule or in a rush to get somewhere, it might be irrelevant. The same traffic jam might be benign-positive if it means being stuck on the bus with an old friend one has not seen in a long time, or stressful if it occurs when one is late for work.

If an event is stressful, it furthermore falls into one or more of three categories: harm/loss, threat, and challenge. An example of a harm/loss stressor might be a bereavement, an injury, being laid off, or another experience an individual assesses as a loss or harm. Threat stressors may hold the potential for harm/loss, such as the diagnosis of a disease, an argument with one's work supervisor, or other situations or events that might lead to negative consequences, but in some cases might also be prevented or lessened now that one is aware of the threat. Challenge stressors are unique in that (like threat stressors) they also focus on the potential consequences, but in this case, the consequences may be positive once the stressor has been dealt with, such as personal growth or other gains (Lazarus \& Folkman, 1984). For example, getting a new promotion at work might be stressful, but if one successfully makes the transition and does well at the new tasks, the potential for added income and future advancement is likely positive. Similarly, the stressors involved in planning a wedding or training for a marathon could be appraised as challenge stressors because they lead to a desired goal. One event can have multiple meanings to an individual; thus threat and challenge appraisals are not mutually exclusive, and each of the events described above could be appraised as one or more types of stressor at different points in time (Lazarus \& Folkman, 1984). 
The next step in the process, as seen in Figure 1, is secondary appraisal. This is the point at which individuals assess what they can, should, and will do in response to the stressor (Lazarus \& Folkman, 1984). This involves individual assessments of one's capabilities, as well as the likely effectiveness and results of various potential courses of actions. To return to the traffic jam example, once it has been appraised as a stressful situation, one might consider taking an alternate route, assuming it is possible to do so and that the alternate route is believed to be faster (i.e., not likely to also be clogged by traffic). Secondary appraisal is also the stage at which an individual chooses a coping strategy, in this case perhaps adopting the active coping method of taking the alternate route. Once an attempt at coping has been made or other new information about the situation has been received, the appraisal process begins again, this time referred to as reappraisal. The reappraisal allows the individual to re-examine the situation and determine whether or not things have changed, potentially generating a reappraisal that is different from the initial appraisal of the situation. For example, if the traffic jam clears with time to spare, or the strategy of taking an alternate route is successful, the situation might now be reappraised as irrelevant.

Lazarus also extended the appraisal process to include emotional reactions, and held that emotion and appraisal were innately intertwined (Lazarus, 1999). According to this perspective, emotions are the result of appraisals, or evaluations, of situations, stressful or otherwise. For instance, a situation that is appraised as threatening might produce fear or anxiety, or if the situation gets resolved such that the threat does not come to pass, the appraisal of the state of affairs at that point might produce relief or happiness. Assessing a situation in which an individual judges that someone else has 
something that she wants might result in jealousy, whereas her assessment of a situation in which she feels she has accomplished something important might result in pride. Regardless of whether or not such appraisals are the source of all emotion as some contend (Lazarus, 1999), clearly some emotional reactions may be related to appraisals.

Appraisals themselves may be affected by a number of things. The demographic, social, and personality characteristics sometimes examined as predictors of stressors and their outcomes may also influence the stress and coping process through their effects on appraisal (Almeida \& Horn, 2004). For instance, a major illness diagnosis might be considered more of a threat by a young person than an older adult, a person high in agreeableness might appraise an interpersonal conflict as more of a threat than someone lower in that trait, and someone whose financial situation is already unstable might be more threatened by a financial stressor than someone who is relatively economically secure. It is the nature of the transactional model that these individual-level characteristics influence the process throughout, as do features of the situation or environment. These considerations are built into the larger model of the stress process laid out in Figure 2.

\section{Coping}

The other major component of the transactional model is coping. At this point in the transactional model, individuals act in response to an appraised (or reappraised) situation. Coping may lead to an emotional response, or may be influenced by emotional states, or both, in a "dynamic mutually reciprocal relationship" (Folkman \& Lazarus, 1988, p. 311). It has sometimes been divided into two approaches-problem-focused and emotion-focused (Carver \& Scheier, 1994). In problem-focused coping, an individual's 
attempts at changing a situation or its impact are directed at the problem itself, for instance trying to talk through a disagreement with a spouse. In emotion-focused coping, the focus is the individual's own negative feelings about the situation, for instance attempting to calm oneself down while waiting for test results at the doctor's office. More recent research has separated out numerous distinct coping strategies beyond the relatively simple problem/emotion-focused dichotomy, some of them behavioral, such as coping by use of substances (e.g., drinking to cope), as well as the positively-focused techniques of acceptance, positive reframing, humor, use of instrumental support, and use of social support (Carver, Scheier, \& Weintraub, 1989).

Opinions of how to judge the success or efficacy of the coping process vary widely (Carver \& Scheier, 1994; Folkman \& Lazarus, 1988), and there are numerous ways in which it might be viewed as successful or unsuccessful. Something that might be a successful strategy in one situation, for instance engaging in avoidant coping to improve one's mood by going shopping while waiting for biopsy results, might be unsuccessful in another, such as taking the same shopping trip when one is trying to avoid thinking about one's financial problems. One way in which coping might be considered successful is if the distress caused by the original situation is alleviated or removed, possibly even replaced with positive affect. To return to the traffic jam example, if taking an alternate route successfully gets the individual to work on time, she might be proud of herself for making a good choice, relieved to avoid probable negative consequences, or happy to have found a new way to get to the office when traffic on her usual route is heavy. 
Other possible beneficial outcomes of coping that could be viewed as successes might include the reappraisal of a threat situation as a challenge, in which case the successful completion of the challenge might produce feelings of mastery or accomplishment. An event may also be reappraised as positive after new information arises, or after the individual's attempt at coping has taken place (Folkman \& Moskowitz, 2000). Because of the inherently individualized nature of the transactional model, thorough examination of the stress and coping process under this framework necessitates repeated measures and within-person analysis to gain a more clear understanding of these person-environment interactions and the ways in which stressors may influence individuals through them.

\section{Influences or Effects of Stressors}

Psychological effects of stress in the short term include changes in attention and cognition (Hancock \& Warm, 1989; Mandler, 1993), mood (Eckenrode, 1984; Zautra, 2003), and behavior (Lemke et al., 2007) (behavior is addressed in more detail in Chapter 4), and longer term may include increased susceptibility to psychological disorders (Billings \& Moos, 1982; Hartley et al., 2007). Stressors may also lead to changes in coping strategies and abilities over time as individuals both gather new information about the success or failure of various strategies and situations and perhaps adjust their selfbeliefs accordingly. Changes in mood are primarily seen as increases in negative mood (Affleck, Tennen, Urrows, \& Higgins, 1994; Bolger et al., 1989; Eckenrode, 1984; Stone $\&$ Neale, 1984). For instance, using a daily stressor checklist, Almeida, Wethington, and Kessler (2002) found significant correlations between multiple items from their checklist, 
particularly interpersonal and network stressors, and increases in negative mood, as well as increases in physical health symptoms.

Numerous studies draw links between stressors of varying magnitude and psychological symptoms (Hartley et al., 2007; Pillow et al., 1996; Serido, Almeida, \& Wethington, 2004; Wagner et al., 1988). Stress is also implicated in the development of psychiatric illnesses including mood disorders and post-traumatic stress disorder (Billings \& Moos, 1982; Hartley et al., 2007; McEwen \& Mendelson, 1993; Rabkin, 1993). In fact, Sherbourne, Edelen, Zhou, Bird, Duan, and Wells (2008) found that a reduction in stressors, although not a planned target of the treatment, was one of the pathways through which short-term quality improvement treatments for depression seemed to function.

Studies focused on the physiological effects of stressors have also found links between events and physical symptoms (Mallers, Almeida, \& Neupert, 2005), as well as short-term susceptibility to infectious disease and effects on the healing process of wounds (Stein \& Miller, 1993; S. Cohen \& Hamrick, 2003; Zautra, 2003). Longer-term physiological effects of stress may include increased susceptibility to disease, particularly if the stress is chronic and ill-adapted to or the deactivation of short-term changes is slow or incomplete, leading to allostatic load (McEwen, 1998b).

Research on the effects of stressors has thus encompassed both immediate and long-term effects, with immediate typically measured within minutes, hours, or days of an event, and long-term measures ranging from months to years. Such studies typically examine different types of predictors, with immediate effects studies frequently incorporating daily hassles and more commonly encountered stressors, and longer-term studies typically focusing instead on longer-lasting stress levels or considering a given 
major life event as one of many risk factors (Rabkin, 1993). It seems likely that the main reason for leaving out non-chronic hassles in longer-term studies is because of the challenge of attributing a later outcome to them, given the numerous potential alternate causes an individual might encounter between the two. Attributing later depression, for instance, to a bereavement or to chronic work stress seems much more plausible than attributing it to a long-ago traffic jam. However if an individual's reaction to a traffic jam forms part of a broader pattern of reactivity that is repeatedly occurring in response to such hassles, then it seems plausible that reactivity to those stressors could be implicated in later depression without the need for a chronic stressor or major event. Without longitudinal studies that incorporate hassles and more common events, the longer-term impact of those stressors remains unknown. Current approaches that tend to study immediate and longer-term outcomes of stressors separately have thus left us with an incomplete picture. Bringing the two approaches together as I have done here could allow us to begin unpacking the relationship between immediate and longer-term outcomes of stressors, and thereby learn more about potential pathways for those effects. As laid out in this chapter, the study of stressors includes not only the occurrence or absence of stressors themselves, but also an examination of the individual and situational factors that might affect the results of those stressors. Individual differences such as personality or demographics, individuals' appraisals of potential stressors, features of those stressors, and individuals' immediate reactions to stressors could each play a role in the process of determining what, if any, longer-term consequences a given stressor has for an individual's health or well-being. This set of possible conceptual 
relationships is drawn in Figure 2. In the next chapter, I will examine the topic of immediate reactions to stressors in more depth, with a specific focus on reactivity. 


\section{Chapter 3: Reactivity}

Reactivity can be defined as the extent to which individuals respond behaviorally, emotionally, or physically to stressors. It represents another way, aside from varying appraisals, in which two individuals might respond differently to the same stressor. An individual's level of reactivity might be low, demonstrated for instance by small behavioral changes, a low-intensity emotional experience, or a minor change in heart rate, or high, demonstrated by greater behavioral change, a high-intensity emotional experience, or larger changes in heart rate. For example, two nurses, both faced with an unusually heavy workload because a colleague has called in sick, might appraise the situation in the same way and still have different levels of reaction to it. One might become frustrated to the point that it changes the tenor of her entire shift, possibly even leading her to drink more alcohol than usual after her shift to cope with the negative emotion, whereas the frustration the other experiences might be fleeting and mild in comparison. If this pattern were relatively consistent over time, the first nurse might be described as more reactive than the second.

Although reactivity is often viewed as a stable individual difference, evidence suggests that there are also dynamic factors at work. Individuals likely have an innate level of reactivity (Belsky \& Pluess, 2009), but that level can be changed by events (Lepore et al., 1997) and can also vary with life circumstances and individual situations (Neupert et al., 2007; Sliwinski et al., 2009). This tendency to react may then be observed through changes in health behaviors such as drinking, eating, and exercise when stressors occur, as well as through self-reported changes in emotion and measured changes in physical indicators. This dissertation focuses on psychological reactivity as 
demonstrated through behavioral changes; in this literature review I draw on some studies of other measures of reactivity when similar studies with behavioral measures are not available.

Although the study of reactivity has been largely atheoretical to date, I have conceptualized it for the purposes of this dissertation as fitting into the transactional model of stress and coping, described in the previous chapter. Different types of reactivity might fit into that model in different ways. Physiological reactivity in the form of changes in heart rate or hormonal levels could affect individual perception of one's own internal state and thus impact appraisals. Emotional reactivity could similarly affect appraisals; for instance a person who is more emotionally reactive could potentially be more likely to appraise the same situation as more stressful than someone who is less emotionally reactive might. Behavioral reactivity would then take place within the coping portion of the process, as individuals either engage in coping-motivated health behaviors (e.g., eating, exercise, alcohol consumption) in response to a perceived stressor, or "use up" their self-control through coping in other ways, resulting in behavioral reactivity; both of these possibilities are discussed in more detail in Chapter 4 . Other broad conceptualizations of the stress and coping process, such as resource-based theories (Hobfoll, 1989, 2001), do not from my perspective allow for as natural an integration of all types of reactivity, particularly incorporating the ideas of both innate and situationally variable levels of reactivity and of coping-related reactivity as the transactional model does. Thus the transactional model is the framework from within which I have approached the topic. 
The consequences of reactivity are not yet fully understood, and its longitudinal linkages to outcomes are largely unexplored. Most studies of psychological reactivity focus on its short-term or immediate effects, but there are multiple ways in which it might have an effect on individuals' longer-term health and well-being. In this chapter, I will first address the ways in which reactivity is conceptualized in the literature, and then the ways in which it might tie to outcomes.

\section{Reactivity and its Individual-Level Correlates}

Reactivity is often discussed as a fairly stable individual difference variable, similar to but distinct from personality. There is, in fact, evidence that individual variation in how likely one is to be affected by positive or negative environmental factors (i.e., reactivity) may be partly determined by genetics (Belsky \& Pluess, 2009). There are also links between the study of psychological reactivity and literatures on emotion regulation (Eisenberg et al., 1993; Eisenberg \& Fabes, 1992; Fabes \& Eisenberg, 1997; Salovey, Hsee, \& Mayer, 1993) and temperament (Derryberry \& Rothbart, 1988), in that dispositional levels of arousability or trait ability to self-regulate one's negative moods and emotions might influence individuals' measurable levels of reaction to stressors. This suggests that reactivity may be at least partly innate. However, evidence has also shown that reactivity may be subject to change across the lifespan, with longitudinal studies suggesting greater reactivity over time (Mroczek \& Almeida, 2004; Sliwinski et al., 2009), and a cross-sectional study finding lower reactivity associated with age (Neupert et al., 2007). Reactivity has also been found to change in response to life events (Lepore et al., 1997). Both of these findings suggest that even if reactivity is relatively stable in general, it is not necessarily fixed throughout the lifespan. 
In addition to being an inter-individual difference, reactivity is a potential source of intra-individual variability. In other words, reactivity both varies across individuals, and can contribute to variation within each individual's behavior. A person who is more reactive, for instance, would be more likely to exhibit larger changes in mood or behavior when encountering stressors than someone who is less reactive. Therefore an individual who is more reactive and encounters many stressors might display a large amount of short-term variability, whereas someone who is less reactive and encounters the same number and type of stressors might not.

There has been increasing recognition that sources of intra-individual variability are worthy of deeper examination as the exploration of various psychological processes continues. Nesselroade (2004; Nesselroade \& Salthouse, 2004) has called for further study of the phenomenon of intra-individual variability, both that involved in change or growth over time and that involved in short-term variations in reactions or performance, pointing out that such variability has been demonstrated to have predictive power of its own for diverse outcomes such as age-related cognitive decline, depression, and perceptual-motor performance. Thus one-time measurements, which by definition cannot capture intra-individual variability, would be insufficient to obtain an accurate representation of an individual's actual pattern of those constructs. In other words, an identical score for two individuals on a motor task might represent one person's best performance and another's worst, making it appear as though their performance is similar when that would seldom actually be the case. Thus, depending on the construct of interest, repeated measures may be necessary. Otherwise, Nesselroade has argued, there 
is a risk of confusing multiple sources of variation, such as cross-sectional differences, individual change, practice effects, and measurement error.

Mischel approached the issue of intra-individual variability from the perspective of personality, in which apparent inconsistencies in behavior across situations might call into question the idea that individual characteristics produce consistent and stable patterns of behavior (Mischel \& Shoda, 1995; Shoda, Mischel, \& Wright, 1994). For instance, a person might behave in an outgoing way at one party, but remain withdrawn at another. To resolve this he has proposed that the individual's transactions with the environment are stable, and that examining the broader situational context in which behaviors take place can allow for predictability. For instance the partygoer might be outgoing at parties attended by friends, but withdrawn at parties attended by strangers. Reactivity could, like personality, be contextualized in environmental transactions, more pronounced at some times than others.

Some studies have drawn a direct connection between reactivity and personality, typically finding that those higher in neuroticism may also be more reactive (Bolger \& Zuckerman, 1995; Suls \& Martin, 2005). Specifically, those scoring higher on neuroticism have been found to report more stressors, and to be more emotionally reactive to the stressors they report. This may be particularly true for interpersonal stressors (Bolger \& Schilling, 1991). Moreover, the greater reactivity to stressors found in those scoring high on neuroticism seems to be more strongly linked to psychological distress than their greater exposure to stressors (Bolger \& Zuckerman, 1995).

Despite their association, however, neuroticism and reactivity remain distinct constructs. Neuroticism is associated with reactivity to negative events, but not positive 
ones (Suls \& Martin, 2005), and in one study, when reactivity was measured physiologically, those scoring higher in neuroticism were not significantly different in reactivity from those who scored lower (Schwebel \& Suls, 1999). This suggests that although the two constructs are related, they are not one and the same. They might, therefore, be expected to be correlated but make distinct contributions to the prediction of outcomes.

\section{Situational/Dynamic Influences on Reactivity}

In addition to dispositional factors, situational factors may influence reactivity. Such factors could relate to the broader contexts of individuals' lives, or to specifics of the situation at hand. In one study, perceived control over one's circumstances, which could factor into appraisal of events, was found to be related to reactivity, with those who believed more strongly that they were in control of their lives demonstrating less reactivity to stressors (Neupert et al., 2007). Although that finding was for a more general sense of control, it seems likely that sense of control varies across domains and situations, suggesting that individuals might be less reactive to parts of their lives where they feel more in control. As discussed in Chapter 2, control is also a component of multiple models of work stress. Contextual factors, on the other hand, might include larger life events with which individuals are dealing, for instance, or their level of psychological well-being. Greater levels of global stress, for instance, have been found to be associated with greater reactivity (Sliwinski et al., 2009).

Similarly, those who are currently experiencing symptoms of depression, which can come and go over time, have been found to respond differently to social interactionrelated events in their daily lives (Peeters, Nicolson, Berkhof, Delespaul, \& deVries, 
2003; Steger \& Kashdan, 2009). One study found that those who were depressed were more reactive to daily events and reported more negative events and fewer positive ones; this is consistent with the conceptualization of an episode of depression as a period of low positive affect and high negative affect, which might be triggered by such events (Steger \& Kashdan, 2009). However an earlier study found that those who currently had a diagnosis of major depression reported fewer positive but no more negative events, and were not more reactive to negative events (Peeters et al., 2003). The exact influence of depressed mood on reactivity and events, therefore, remains unclear. These broader life situations may be affecting how individuals interact with their environments, perhaps by being more or less attuned to events of a certain type when they occur, by recalling and reporting them differentially later, or by engaging with their environment in a way that causes more negative or positive events to occur.

These person-environment transactions may also be seen through the lens of the transactional model of stress and coping discussed in Chapter 2. Under transactional models of stress and coping, reactivity to stressors could play a role in determining whether or not a given potential stressor is actually perceived as negative. An individual who reacts relatively more intensely to relatively less extreme stimuli ("flies off the handle easily," colloquially), or who is already feeling distressed when an event takes place ("the last straw"), might react strongly to even apparently small stressors that might not distress someone who is less reactive or someone who is in another emotional state (Suls \& Martin, 2005). These more noticeable responses to stressors may produce more easily measurable results. 
The situational and individual differences perspectives on reactivity can work together as well. To return to the example given earlier in this chapter of two nurses who both experience the stressor of an unusually heavy workload because a colleague has called in sick, a within-person examination of these two nurses could reveal a variety of patterns. As described previously, both nurses reacted negatively to the stressor, but one nurse was more reactive to that particular situation than the other was. Yet that may not be their consistent pattern over time. For instance, the nurse who was less frustrated in that example could be a person who is less reactive than her colleague all the time, even in the face of constant exposure to stressors. Alternately, she could be a person whose exposure and reactivity to stressors is highly variable, with that one instance of low reactivity representing one end of the range of her typical pattern, whereas her colleague who experienced greater frustration in that situation might be less variable in her level of response. Their individual patterns of reactivity on any given workday could vary because they have different innate levels of reactivity, different levels of investment in their job, or both, or it could relate to some other factor influencing their individual levels of reactivity from their life contexts, individual differences, or the situation itself. This reactivity might then be demonstrated by individual behavioral changes, such as drinking to cope or stress-related eating.

\section{Behavioral Reactivity}

Psychological measures of reactivity often include changes in mood, but those changes may be fleeting and thus quite difficult to capture empirically, so behavioral changes that may be stress-related have also been considered as measures of reactivity (Lemke et al., 2007). Several possible changes in health behavior (e.g., exercise, eating, 
alcohol consumption) that can serve as measures of behavioral reactivity are discussed in greater depth in Chapter 4. These measures can be obtained in a naturalistic setting through within-person diary studies. Researchers are still working to understand how and if different types of reactivity (e.g., physiological, psychological, behavioral) relate to one another and to different types of stressors (Lepore et al., 1997). It is, however, clear, that encountering stressors can influence an individual's behavior.

Although not directly conceptualized as reactivity in all cases, numerous studies have demonstrated links between negative events or emotions and immediate changes in behavior, such as alcohol consumption and eating. In one investigation, daily hassles were found to be correlated to greater snacking in a two-week daily study of women (Newman et al., 2007). Motivation to eat has also been found to increase when individuals are experiencing negative emotions, as has experience of hunger (Macht \& Simons, 2000). One retrospective study suggested that individuals increased their alcohol consumption in response to stressor exposure, specifically conceptualizing this increase in drinking as behavioral reactivity (Lemke et al., 2007). Mohr et al. (2001) found an association between increased solitary drinking and negative interpersonal experiences. These specific examples, most of them conceptualizing stressor-related health behavior change as an attempt at coping, are only a few of the studies that have linked negative events, experiences, or emotions to immediate behavioral change. The effects of these behavioral changes may be one way in which reactivity could lead to negative outcomes.

\section{Effects of Reactivity}


Reactivity could play a role in the pathways from stressors to consequences in multiple ways - those who are more reactive to stressors may be less likely to cope well with them (Fabes \& Eisenberg, 1997) or to recover quickly and fully from the physiological effects of stressors, and therefore may be more likely to experience stressrelated physical and psychological distress, particularly if the stressors are chronic (Lepore et al., 1997). Moreover, behavioral manifestations of reactivity, such as changes in health behaviors, can have their own direct consequences; for example, drinking to cope may be associated with greater likelihood of developing alcohol problems (Cooper, Russell, \& George, 1988). Individuals who overeat or eat unhealthy foods in response to stressors might be susceptible to diet-related health consequences (Liebman, 2009). Although reactivity is most often conceptualized as a negative response, behavioral reactivity could potentially be positive and lead to positive consequences for some types of behavior and directions of change. For example, those who react to stressors with increased exercise are likely to receive both physical and mental health benefits (Petruzzello, Landers, Hatfield, Kubitz, \& Salazar, 1991; Thayer, Newman, \& McClain, 1994; Thayer, 1987), whereas those who reduce their physical activity in response to stressors would receive a lower level of these exercise-related health benefits than usual.

Within the context of work, reactivity also has the potential to affect outcomes of work stressors. Most studies of reactivity in the work environment have focused on physiological reactivity and outcomes. Cardiovascular reactivity among air traffic controllers has been found to be associated with later development of hypertension, for instance (Ming et al., 2004). Karasek's stress disequilibrium theory (2008), rooted in his work on job demands and control, proposes that the body's attempts to restore 
equilibrium after physiological reactivity both exhaust and create wear and tear on physiological systems that would ordinarily assist in the prevention of disease. This theory and the research on physiological reactivity to work stress are both consistent with the principle of allostatic load, described earlier. However the effects of psychological reactivity on health in workers have been less clear. One study, for instance, found that emotional reactivity was, contrary to the researchers' expectations, not associated with later cholesterol levels, with one exception — a significant interaction of burnout and reactivity in which those who were less reactive and more burned out had higher cholesterol at follow-up than those who were more reactive and more burned out (Shirom, Westman, Shamai, \& Carel, 1997). In other words, greater emotional reactivity may have had a protective effect against cholesterol. Studies using behavioral measures of reactivity in a work context are uncommon. In one study that was not focused directly on work reactivity but included diary measurement of events from multiple domains, Carney, Armeli, Tennen, Affleck, and O’Neil (2000) found that negative work events were associated with greater desire to drink. This suggests a possible link between work stressors and behavioral reactivity.

Regardless of how it is measured, or whether it is conceptualized as a stable individual difference or a situationally variable factor, reactivity to events at work has the potential to influence nurses' health and well-being through multiple pathways, including stress-related changes in health-impacting behaviors such as alcohol consumption and exercise. 
Chapter 4: Behavioral Reactivity, Health, and Well-Being

Individuals' reactivity to stressors may take the form of changes in health behaviors such as drinking or eating, as discussed in the previous chapter. Someone might drink more or less alcohol or exercise more or less in response to stressors, possibly as an attempt at coping with stress-related negative emotions. In addition to the idea of health behavior change as an attempt at coping, recent research has offered another theoretical context for why such changes might occur under stressful circumstances. Muraven and colleagues (Muraven \& Baumeister, 2000; Muraven, Tice, \& Baumeister, 1998) put forth the idea that self-control is a limited resource that can be temporarily depleted. They have tested this with a variety of laboratory models that use cognitive, emotional, and physical tasks, including emotion regulation in response to an upsetting film. Prior to this, researchers examining how individuals' capabilities change in response to stressors had described stress-related limitations on the functional resources of individuals (Hockey, 1984), or on mental control as a whole (Wegner \& Pennebaker, 1993), more general propositions that are in line with Muraven and colleagues' more specific description of self-control limitations.

If self-control is indeed a limited resource, then while one is coping with stressors, self-control in other arenas may be impaired, leading to deviations from individuals' usual level of self-regulation. One example of this comes from the O'Connor and O'Connor (2004) study in which women who were attempting to diet were more likely to engage in stress-related eating than those who were not. During a stressful week, the self-control required to engage in regular exercise, eat healthy foods, or keep drinking to a moderate level might have been "used up" on the stressors, leaving 
individuals more likely to give in to whatever temptations they might normally resist. This in turn has both short- and long-term effects on health, as alcohol consumption, exercise, and diet each have the potential to affect individuals both immediately upon engaging in those behaviors, and later on through longer-term patterns of behavior. Thus behavioral reactivity can result from both stressor-related self-control failure and behavior changes that are an attempt at coping with stressors.

Many of the physical and mental health outcomes commonly studied in stress and coping research, such as cardiovascular health and psychological well-being, can be influenced not only by physical and neurochemical effects of stressors and emotional reactions to events, but also by the choices individuals make about health behaviors. These choices may in turn be affected by individuals' reactions to positive and negative events; in fact changes in health behavior have been described as "among the earliest and most overt signs of rising levels of stress," (J. D. Quick, Horn, \& Quick, 1987). Therefore there are multiple mechanisms, both direct and indirect, through which stressors and positive events may affect health. In this dissertation I chose to focus on three types of common volitional health behaviors that seem likely to be variable across and within individuals, and that may be affected by stressors. Specifically, I examined alcohol consumption, dietary choices, and exercise. These three behaviors can be influenced by events and can have an influence of their own on health in the short and long term. Following through on this approach to studying behavioral reactivity and outcomes, future studies could examine these behaviors in more depth and a number of less common and perhaps less intra-individually variable health behaviors, such as cigarette smoking, could also be considered. 


\section{Health Behavior}

Alcohol. Although moderate drinking may have some beneficial health effects, such as the reduced risk of mortality seen among older women in the Nurses' Health Study (Colditz et al., 1997), heavy drinking can be harmful to health, and the impaired judgment that goes along with drinking to intoxication can lead to serious consequences (Ashley et al., 1997; Colditz et al., 1997). Moreover, self-reported drinking to cope with negative emotions has been shown to be linked to alcohol problems (Cooper et al., 1988). Therefore researchers have investigated both the motivations for and consequences of alcohol use and abuse.

The tension-reduction hypothesis was put forth by Conger (1956), who, grounded in behavioral theory, suggested that drinking behavior could be reinforced if its inhibition-lowering effects allowed an individual to reduce a drive (such as sexual desire), or if it reduced unpleasant experiences of fear or conflict. Modern research continues to draw on the idea that alcohol use might be reinforced if it reduces unpleasant feelings or experiences (e.g., Cooper et al., 1995), and thus drinking might function as a coping technique.

Cox and Klinger (1988) described individuals' expectations about alcohol use as a key part of their decision-making process about drinking, with their belief that it would either enhance mood or reduce tension as a key part of their motivation to drink. Motivational models were further expanded by Cooper, Russell, Skinner, and Windle (1992), who were able to provide evidence that drinking to cope with negative emotion, drinking to enhance positive emotion, and social drinking were distinct from one another. Individuals, therefore, may drink for different reasons at different times. 
Based on this body of research, one might posit that individuals would drink more when they encounter stressors; and although that pattern has frequently been found, the exact relationship between stressors and drinking has proven to be complex (Mohr, Armeli, Tennen, \& Todd, 2010). Within-person analyses of diary data, in which individuals record their stressors and drinking repeatedly over time, often at a fixed interval such as at the end of each day, have shed some light on these connections. Both the type of stressor (Carney et al., 2000) and the context of the drinking (Mohr et al., 2001) have been shown to be important, and specific (discrete) negative moods may have different effects (Mohr et al., 2008). Investigating drinking-to-cope in the work context, Liu, Wang, Zhan, and Shi (2009) found that increased daily alcohol use and desire to drink were both predicted by greater work stress. Moreover, those who scored higher on neuroticism and on job involvement demonstrated a greater increase in drinking. Frone (1999) reviewed research on work stress and drinking and suggested a conceptual model in which the effect of work stress on drinking is moderated by protective or vulnerability factors such as placing a particularly high importance on work, and mediated by additional factors such as depression. Measures of work stressors, however, were found to be relatively lacking in diversity in much of the literature, and longitudinal studies where changes in drinking could be examined over time were relatively rare. In this dissertation I have attempted to help address both of those gaps by including a variety of work stressors as well as longitudinal measures of health.

Diary studies attempt to reduce the amount of time between events, feelings, behavior, and their assessment as much as possible (thereby reducing recall biases); they also allow untangling of temporal ordering that might not be possible retrospectively. 
Moreover, an individual might not be consciously aware that her behavior changed in response to a particular event; a diary measure that asks about both events and behaviors can capture the linkage between them regardless of whether or not that link was in conscious awareness. One study comparing a one-month retrospective measure of drinking to daily measures found that although the retrospective measures captured the overall level of drinking relatively well, within-person fluctuation was better captured by the shorter timeframe of recall (Carney, Tennen, Affleck, Del Boca, \& Kranzler, 1998). Within-person diary-based investigations have begun to shed light on the relationship between stressors and drinking. In a study of moderate to heavy drinkers drawn from the community, Mohr et al. (2001) found that negative interpersonal experiences were associated with solitary drinking, whereas positive interpersonal experiences were associated with social drinking, suggesting patterns of drinking to cope with negative experiences and enhance positive ones. By carefully timing the collection of data about events and drinking, within-person patterns of stressors and behavior can emerge.

Diary studies can also encompass slightly longer time frames, as Totterdell, Wood, and Wall (2006) did in their use of a weekly diary to study strain in a sample of self-employed workers. They examined the job demands-control model on a weekly, intra-individual level and found that weekly patterns of demands, control, and strain varied from person to person, depending in part on individual characteristics. Thus a within-person analysis that took into account inter-individual differences was necessary. Patterns captured with diaries can have implications for later outcomes, as in a study of moderate drinkers by Mohr et al. (2013; Mohr, Brannan, Wendt, Jacobs, \& Wright, 2010). In that study, our research team used the statistical technique of slopes- 
as-predictors (described more fully in Chapter 5) to examine the predictive power of daily mood-drinking associations. Specifically, daily negative mood-drinking home alone associations, typically conceptualized as drinking to cope, were associated with generally better outcomes one year later, including increased satisfaction with life. This result is contrary to the typical conclusion that drinking to cope is associated with a greater risk of developing alcohol problems; however for most of the adults in the study, the actual number of drinks consumed was still within the normal recommended limits. Thus it seems possible that for that particular sample of relatively psychologically healthy, moderate-drinking adults who displayed a relatively small magnitude of behavioral reactivity, drinking to cope may in fact have been a successful strategy. This use of behavioral reactivity as a predictor of outcomes adds a new dimension to withinperson examination of events and drinking.

The effort to understand the relationships between events, emotions, and drinking is still ongoing, but what is clear is that many individual and situational variables can play a role, and that moreover, within-person analyses often reveal different patterns of results from between-person analyses (Armeli, Todd, \& Mohr, 2005). Because reactivity is demonstrated by stressor-related changes in individual-level behaviors such as drinking, within-person techniques are similarly necessary for the study of behavioral reactivity.

Exercise and diet. Exercise has many health benefits. In addition to improved physical health, exercise is beneficial to psychological well-being and specifically to mood (Centers for Disease Control and Prevention, 2011; Petruzzello et al., 1991; Thayer et al., 1994; Thayer, 1987). Moreover, lack of exercise, or physical inactivity, has been 
estimated to cause $9 \%$ of premature mortality worldwide (Lee et al., 2012). Thus exercise has both short-term (mood) and long-term (physical health) benefits. Some individuals may take advantage of the benefits of exercise on mood by using it as a means of coping with stressors. Alternately, individuals who find it challenging to maintain regular exercise habits might find it difficult to maintain those behaviors when faced with stressors, as mentioned in the discussion of self-control above. Evidence consistent with this was found in a daily diary study conducted by Payne, Jones, and Harris (2010), in which individuals' intentions to exercise were found to be less likely to be carried out on days when their job demands were higher. An earlier weekly diary study also found similar results; participants tended to exercise for less time during weeks with more stressful events, and on fewer days during weeks with greater perceived stress (Stetson, Rahn, Dubbert, Wilner, \& Mercury, 1997). Therefore, individuals might respond to stressors by exercising either less than usual, if the self-control to maintain their usual schedule has failed, or more than usual, if they are engaging in exercise as a coping strategy. Exercise as a coping strategy is not as well-researched as some other techniques, but factor analysis of exercise motivations has revealed that coping is one of four groupings of core reasons why women engage in exercise (Cash, Novy, \& Grant, 1994).

Although it has been hypothesized that one mechanism through which improved mood might occur in response to exercise is decreased physiological reactivity because of increased cardiovascular fitness, a meta-analysis found that if such a change exists, it is of small magnitude and compromised by methodological problems in the studies being examined (Dishman \& Jackson, 2000). Therefore it remains unclear whether exercise 
affects the stress process itself (i.e., reactivity) as well as outcomes. It is possible that exercise, and the positive moods it generates, could act as a resource that increases wellbeing and improves future coping, a process referred to as "broaden-and-build" (Fredrickson, 1998, 2001; Tugade, Fredrickson, \& Feldman Barrett, 2004). Whether or not that is the case, based on the mood benefits of exercise alone, those who engage in exercise during stressful periods of time seem likely to fare better in the short term than those who do not.

Dietary choices also contribute to overall health, and eating too much food or poorly chosen foods can increase risk for a number of health problems over time (Liebman, 2009). Examples include the links between trans fats and coronary heart disease and red meat and cancer found by the NHS (Colditz et al., 1997). Snack choices are a particular area of focus in research. In addition to longer-term health problems, sugary snacks have been linked to short-term negative changes in mood and to later decreases in energy (Thayer, 1987). Therefore changes in diet can also have effects in both the short (mood, energy) and long term (health problems).

Numerous studies have demonstrated that exposure to stressors and negative emotions can change individuals' eating behavior, often for the worse (Macht \& Simons, 2000; Newman et al., 2007; O’Connor \& O’Connor, 2004). Macht and Simons (2000) found that negative emotion was associated with eating-to-cope, and Newman et al. (2007) found that daily hassles were related to snacking. However, emotion and daily events are not the only factors involved in stress-related changes in eating. O'Connor and O’Connor (2004) examined stressful and non-stressful periods of time, and found that stress-related eating also had links to individual differences such as 
conscientiousness. Thus some individuals may engage in stress-related eating, whereas others do not. In one study of nurses and teachers that compared high- and low-stress weeks to one another (Steptoe, Lipsey, \& Wardle, 1998), some participants drank to cope, and some ate more fast food, cheese, or sugary snacks during the high-stress weeks as compared to the low-stress weeks. Thus individuals may differ in their patterns and types of stressor-related changes in eating.

As described above, there are a number of ways in which health behaviors may change in response to stressors. Some studies, in fact, have linked health behavior to reactivity, and in this dissertation I have followed that example, conceptualizing health behavior change as a measure of reactivity. Using physiological measures of reactivity, Newman, O'Connor, and Conner (2007) found that although their sample as a whole had a positive association between daily hassles and snacking, when the sample was divided into high and low reactivity groups there was no longer a significant association between hassles and snacking for those who had low reactivity. In other words, snacking in response to hassles was only found for those who were also more physically reactive to those hassles. This was in line with previous laboratory findings (Epel, Lapidus, McEwen, \& Brownell, 2001), and suggests that in the absence of physiological measures, eating behavior in response to stressors might be considered as a measure of reactivity. The mechanism, therefore, may be that stressors lead directly to eating, exercising, or drinking to cope, or alternately, because of the self-control used up in coping, stressors might lead to a breakdown in the self-control normally used to keep good exercise, drinking, or dietary habits. Both of these options are laid out in Figure 3. This change, whether initiated as a coping mechanism or through an exhaustion of self-control 
resources, could have longer-term health consequences, particularly if it is a frequent or ongoing pattern for the individual.

In this dissertation, health behavior changes have been conceptualized as a marker of reactivity to negative work events. Specifically, within-person variations in health behavior were examined alongside within-person variability in work stressors. Nurses' reactivity to work stressors, measured by changes in health behavior, may have an effect on later health, health behavior, and well-being.

\section{Well-Being}

As with "health," the term "well-being" is often used in a nonspecific way to refer to an overall state. Some bodies of research have very specific definitions and terminologies for psychological well-being or subjective well-being, while in other cases well-being is used simply to indicate a lack of psychological problems, or sometimes that lack accompanied by a general state of thriving and positive mental health. Well-being is also sometimes interchanged with health and may then be referring to physical state as well as (or instead of) the psychological. In this dissertation, I will focus on three key areas of health and well-being: subjective well-being, which is a way of measuring selfperceived quality of life; depression symptoms; and self-rated physical health. In addition to being relevant to physical health, individuals' self-perceptions of their health have been shown to be correlated to psychological distress (Tessler \& Mechanic, 1978). In other words, poor perceived health may in some cases be a marker for mental health problems as well as physical ones. Among mental health symptoms and problems, depression is a particular area of concern for those working in demanding occupations (e.g., Hartley et al., 2007), and is also associated with job burnout in nurses, which might 
lead them to consider leaving the field (Glass, McKnight, \& Valdimarsdottir, 1993).

Thus it has potential implications for the nursing shortage as well as the well-being of the individual.

Subjective well-being (SWB) is the individual's perception of the quality of his or her life, and has sometimes also been described as "happiness" (Diener, 1984, 2000). More specifically, SWB is made up of general and domain-specific satisfaction with life, as well as low negative affect and high positive affect. SWB measures have been widely used, and have even been proposed for use as a national-level indicator (Diener, 2000, 2006). Although in the moment reports of SWB are subject to the effects of current mood, longitudinal studies have found a fair amount of stability in SWB over time (Lucas, 2008; Suh, Diener, \& Fujita, 1996). In a study that measured life events, Suh et al. (1996) found that the effects of even fairly major events such as job loss on SWB and affect only lasted a few months. Thus SWB seems to be a relatively general measure of overall self-perceived quality of life that tends to be robust over time. Some researchers have sought to bring non-self-report measures into this body of work, such as Nave et al. (2008), who compared self-rated SWB with behavioral observations, clinician judgments, and peers' ratings. They found that overall the self-report measures of SWB lined up relatively well with the other reports.

Work has often been overlooked as a domain of life that might contribute to SWB, and the relationship between work satisfaction and life satisfaction is likely at least somewhat reciprocal, but for those who are engaged in work it may have an impact on SWB (Erdogan, Bauer, Truxillo, \& Mansfield, 2012). In one cross-sectional study, nurses were found to have higher SWB than the general population, but lower job 
satisfaction, and accordingly they had a smaller correlation between job satisfaction and SWB than that found in the general population (Sparks, Corcoran, Nabors, \& Hovanitz, 2005). The authors suggest that nurses might be happier than average when they enter the field, and that they may also derive meaning and happiness from a sense of the importance of their work to society, even if their satisfaction with the job itself is lower than expected.

In addition to affect and satisfaction with life, as measured by SWB, well-being as a broader construct is often characterized by an absence of psychological problems such as depression. Each of these factors may operate in multiple directions, both affecting how the individual interacts with his or her environment and being affected by the individual's environment and experience (Lyubomirsky, King, \& Diener, 2005). Thus an individual's levels of happiness and depression symptoms make up part of the individual context in which the transactional model of stress and coping plays out.

For example, depression and symptoms of depression may both arise at least partly from environmental conditions and, in turn, may then affect those conditions, either directly or through individuals' person-environment transactions. Lewinsohn and Graf (1973) found that engaging in pleasant activities was positively correlated with mood, and that those who were depressed engaged in fewer pleasant activities than control participants did. In this case, the lack of interest that is characteristic of depression might be leading individuals to engage in fewer pleasant activities, which might in turn increase their depression. In a daily study, Nezlek and Gable (2001) found that those who were depressed were more reactive to daily events, both negative and positive. Thus those who are depressed are less likely to be engaging in the positive 
experiences that might help alleviate their depression. The scarcity of positive events seems to be accompanied by a larger amount of negative events, as well. Russell and Cutrona (1991) found that those who were more depressed were likely to report more daily hassles and major negative life events, which then predicted subsequent depression. In cross-sectional studies, it may therefore be difficult to determine whether a greater frequency of negative events led to depression or resulted from depression, although the Russell and Cutrona study suggests that both are likely to occur.

In addition to being a benefit of its own, increased well-being has been linked to a number of positive physical and mental health outcomes, including reduced risk of coronary heart disease (Boehm, Peterson, Kivimaki, \& Kubzansky, 2011; Lyubomirsky et al., 2005). Hassles and uplifts (daily positive events) also contribute to physical and mental health, as seen in Stephens and Pugmire's (2008) study of police officers, in which uplifts seemed to contribute more to physical health, whereas hassles seemed to contribute more to psychological distress.

As described throughout this chapter, there are numerous potential links between stressors, behavioral reactivity, and well-being. It seems plausible that some individuals at some times take part in a cyclic pattern of events, reactivity, and well-being, in which they maintain well-being, experience and react to more positive events, and continue to maintain or improve their well-being, or experience and react to more stressors, have lower well-being, and continue to experience more stressors. One study of depression treatment in fact found support for the idea that improved mental health might reduce incidence of stressful events, which would then in turn facilitate continued recovery from depression (Sherbourne et al., 2008). Experiencing fewer negative events would also 
mean fewer opportunities for reactivity and thus reduced potential for that reactivity to cause harm.

Longitudinal within-person research, therefore, offers the opportunity to examine the experience of and reactivity to negative events, and to link those factors to later wellbeing. Thus this dissertation, in which nurses are followed over time, can help to explicate the relationships between work events, behavioral reactivity to those events, and health and well-being. I measured health and well-being in both psychological and physical domains, using nurses' satisfaction with life and symptoms of depression to examine their psychological well-being, and reports of perceived health to examine physical well-being. 


\section{Chapter 5: The Current Study}

Although it is widely accepted that stressors at work can have negative health consequences for employees, the processes and individual factors involved in determining how, why, for whom, and under which circumstances this occurs are still being investigated. In this dissertation, I put forth the proposition that reactivity, as measured through stress-related changes in health behavior, is part of this process. Specifically, if individuals change their health behaviors when they encounter stressors, those changes themselves may have effects on later health. Direct links from stressors to physiological effects (e.g., allostatic load, see Chapter 2) have been examined previously in research (McEwen, 1998b). The pathway described in this dissertation from stressrelated behavior change to health represents a previously unexplored potential route for the effects of stressors on well-being.

\section{Work Stressors and Health in Nursing}

As discussed in previous chapters, this dissertation uses data gathered as part of the Oregon Nurse Retention Project (ONRP). My role on the ONRP team included, but was not limited to, involvement in the development and selection of questionnaires and scales, focus groups with nurses and pretesting of the survey instruments, data collection, and data cleaning, programming, and analysis.

The ONRP as a whole was framed around the Oregon Nurse Retention Model (ONRM), which sought to integrate the stress and job retention literatures into a model of nurse retention (Sinclair et al., 2009). The ONRM as illustrated in the ONRP final report is displayed in Figure 4. In this model, nurses' positively and negatively appraised work experiences lead to reactions, both positive and negative (specifically engagement and 
burnout), which in turn lead nurses to want to either stay in or leave their job. Combined with a perception of how easy it would be to leave their job, this then leads to retention outcomes, such as looking for another job. These processes are nested in the organizational context and influenced by both that context and the individual's characteristics. This dissertation is focused on the experiences and reactions portion of the ONRM, and on health outcomes rather than job retention outcomes. Therefore the reactions of interest are changes in health behavior rather than engagement or burnout.

Keeping the field of study narrowed to the work environment, and moreover to one occupation, reduces the variability in the stressors participants encounter, as discussed in Chapter 2. This restriction of scope could be seen as creating a kind of natural laboratory in which to study work stress and health among nurses, a population that is likely to encounter many work stressors (Lambert et al., 2003; McNeely, 2005). Furthermore, the focus on those work stressors allows the comparisons to be "apples to apples," examining the effects of nursing work stressors on all participants, rather than, for example, nursing work stressors on some and police work stressors on others. This reduced variability among stressors may help, for the purposes of this initial investigation, to clarify the relationships between stressors, reactivity, and outcomes. Future studies could expand the frame to include other stressors beyond those encountered in nursing.

This dissertation focuses on nurses working in hospital settings for two primary reasons. First, as a population of concern given the nursing shortage, better understanding of the link between nurses' work events and health and well-being may be useful in ongoing attempts to retain nurses in their jobs and the field as a whole. Second, 
as an occupation that includes numerous opportunities for stressors to occur, use of a nursing sample in this dissertation should offer a good sampling of such events, allowing for examination of the relationships between work events, reactivity, and longer-term health outcomes.

\section{Reactivity and Health}

Although studies of immediate reactions to stressors (i.e., reactivity) are increasingly common, few studies have examined the relationships between those immediate reactions and later outcomes, and fewer still have examined reactivity over a longitudinal period. Studies of reactivity as an independent variable are relatively uncommon, and have often utilized cardiovascular or other physiological measures and then used those measures as a trait-like indicator of reactivity when looking at outcomes (e.g., Lepore et al., 1997; Ming et al., 2004). However new statistical methods have provided a way to both measure reactivity across multiple occasions in a real-world setting and use that dynamic measurement as a predictor of outcomes. Specifically, in multiple papers on depression, Cohen, Gunthert, and colleagues (L. H. Cohen et al., 2008, 2005; Gunthert et al., 2005) used the individual-level slopes derived from multilevel modeling of affective reactions to stressors or negative thoughts as predictors in later models.

This approach takes a relationship between two variables as described by a multilevel model (a slope) and uses it as a predictor in a second regression analysis, whereas in a traditional multilevel modeling analysis those slopes might themselves be the outcome of interest, or in a traditional regression analysis a one-time measure of reactivity might be a predictor. Their research group and others have continued to use 
this methodology to study depression etiology and therapy outcomes (Parrish et al., 2011; Wichers et al., 2009). Use of a variation on the same methodology with a community sample of adults revealed that those who had greater daily affective reactivity were more likely to have affective disorders or chronic health problems ten years later (Charles et al., 2013; Piazza et al., 2012). This intriguing new analysis technique, despite its potential usefulness, has yet to be widely adopted in the social sciences. Mohr and colleagues (Mohr et al., 2013) have recently utilized this method with daily mooddrinking slopes, as discussed in the previous chapter; I am unaware of any other studies of drinking or other health behaviors that use this methodology.

In the context of this dissertation, this methodology allows me to determine the strength and direction of an individual's relationship between reactivity and later outcomes. This opens up intriguing possibilities for future research to attempt to untangle the mechanisms by which reactivity relates to those outcomes. If, for example, an individual drinks more in response to stressors, it could relate to longer-term health through two major pathways. The first is the effect of the drinking itself; with past behavior predicting future behavior, drinking more now should be a good predictor of drinking more later on, and over time that drinking may have negative health consequences. The second possibility is that the instability that is inherent to the reactivity itself might be harmful, or be a marker for some other underlying factor that is potentially harmful over time, such as ineffective coping. For instance, avoidance of emotion by coping through substance use can be a predictor of later alcohol problems (Cooper et al., 1988). Short-term intra-individual variability, of which behavioral reactivity might be one representation, can in fact be a powerful predictor of outcomes, 
including risk of mortality among older adults (Nesselroade \& Salthouse, 2004;

Nesselroade, 2004). In cases in which my hypotheses about reactivity predicting outcomes are supported, it seems reasonable that one or both of these two mechanisms could be at work; although it would be premature for this dissertation, which represents an initial investigation of the relatively unexplored relationship between reactivity and outcomes, to attempt to test these underlying processes themselves.

Regardless of the underlying mechanisms that might be at work, individuals whose patterns of reactivity are different, more or less extreme, or even opposite, can be compared to one another through examination of the relationship between those patterns and later outcomes. If someone's health behavior deteriorates more under stress, it seems likely that they might have more long-term negative consequences of this behavior change than someone whose health behavior remains more stable. Conversely, those whose health behaviors improve under stress should show more long-term positive consequences of this behavior change.

\section{Hypotheses}

Behavioral reactivity. In this dissertation, reactivity is conceptualized as change from an individual's typical health behaviors that is linked to variability in their weekly negative work events. In other words, it is not the amount of alcohol consumed that is important to reactivity, but rather whether or not the amount covaries with weekly stressors. If individuals engage in tension-reduction changes in health behaviors (Conger, 1956), or if coping with work stressors uses up the resource of self-control (Muraven \& Baumeister, 2000), then weeks in which nurses experience more negative events than usual might also be weeks in which they engage in poorer health behaviors 
than usual. However some nurses might actually engage in better health behaviors during weeks with more negative events; for instance if they use exercise as a coping method they could be likely to exercise more during those weeks, rather than less. Not all individuals would be expected to respond to stressors with drinking, eating, or exercising to cope; thus individual patterns of reactivity may lead such changes in health behavior to occur for some individuals, but not for others. Therefore I proposed the following hypothesis relating to immediate reactions to work stressors.

Hypothesis 1: There will be significant variance between persons in the weekly relationship between nurses' negative events and their health behaviors.

Specifically this variance will be seen in the relationship between weekly negative events and 1a: days of exercise, $1 \mathrm{~b}$ : minutes per day of exercise, $1 \mathrm{c}$ : healthy eating, $1 \mathrm{~d}$ : number of drinking days, and 1e: number of drinks. Statistically, it takes the form of a significant variance component in the multilevel models.

Hypothesis 1 lays the groundwork for the core hypothesis, Hypothesis 2, in which the relationships between reactivity and outcomes are tested. If there was no significant variance between persons found in an analysis for Hypothesis 1, an exploration of how that variance affects outcomes would not be justified. Such a finding would indicate that there was no evidence of differing patterns of reactivity found within the sample. Thus if Hypothesis 1 failed in all cases, testing of Hypothesis 2 could not proceed.

Reactivity and outcomes. Reactivity has the potential to impact outcomes in a variety of ways, both direct, as in the case of physiological consequences of arousal (S. Cohen \& Hamrick, 2003; McEwen, 1998b), and indirect, through coping (Fabes \& Eisenberg, 1997) or through consequences of the changes in health behavior that may be 
involved in coping (Lemke et al., 2007). Therefore greater reactivity to work stress might lead to poorer health and well-being outcomes later on, or to better outcomes, if the reactivity takes the form of positive changes. This leads to Hypothesis 2 , in which those outcomes are linked to reactivity as measured within nurses' weekly diaries and quantified during the analysis of Hypothesis 1 . This hypothesis attempts to address the core purpose of this dissertation: an examination of the relationship between immediate and longer-term reactions to stressors.

Hypothesis 2: Nurses who show greater weekly reactivity in the form of increased drinking, decreased exercise, or poorer eating habits will have poorer health and well-being outcomes at follow-up; conversely those nurses who show greater weekly reactivity in the form of increased exercise or better eating habits will have better health and well-being outcomes at follow-up.

Specifically, the outcomes measured are 2a: depression scores, 2b: satisfaction with life, and 2c: perceived health. Hypothesis 2, as a test of individual (and therefore variable between persons) patterns of reactivity can determine whether individuals who have similar patterns of reactivity to one another have similar outcomes to one another. The specific expectations for each combination of predictor and outcome are laid out in Table 1. Potential control variables such as age, sex, and neuroticism that are known to be related to some of the outcomes were also considered in the tests of these hypotheses (see Chapter 6).

As described above, increased consumption of alcohol and poorer eating habits could each result from either a failure in self-control because it has been "used up" in coping with stressors or from an attempt to use alcohol or food to cope with stressors. 
Exercising less than usual could also result from self-control failure, whereas exercising more than usual might represent an attempt at coping. Drinking less during times of more stressors might relate to a lack of free time. Eating healthier than usual during times of more stressors seems unlikely given the empirical evidence on stressors and eating described in Chapter 4, but if it occurred in this sample it would be found during the tests of Hypothesis 2, which allow the examination of both positive and negative stressor-health behavior slopes. However, even if no participants eat more healthily during times of more stressors, it seems likely that participants will not universally eat less healthily during such times; some may change their eating habits while others do not.

Depression scores. Prior research has found psychological reactivity to be a vulnerability factor for depression (Charles et al., 2013; Parrish et al., 2011); however this relationship has not been tested for behavioral reactivity. Nurses whose health behaviors are poorer than usual during times of higher stressors (e.g., drink more alcohol than usual, eat less healthily than usual, or exercise less than usual), are therefore hypothesized to have higher depression scores at follow-up. Nurses whose health behaviors are improved during times of higher stressors would conversely be hypothesized to have lower depression scores at follow-up (H2a).

Satisfaction with life. Mohr, Brannan et al. (2010) found that behavioral reactivity in the form of negative mood-drinking home alone slopes predicted greater satisfaction with life at follow-up. However, unlike the Mohr, et al. sample this group of nurses has not been pre-screened to rule out psychological or substance problems, thus I have based my hypotheses about satisfaction with life on the overall pattern of detrimental effects that poor health behaviors can have on well-being, described in 
Chapter 4. I hypothesize that nurses whose health behaviors are poorer than usual during times of higher stressors will have lower satisfaction with life at follow-up. Conversely, those whose health behaviors are improved during times of higher stressors will reap the benefits of those good health behaviors and have greater satisfaction with life at followup $(\mathrm{H} 2 \mathrm{~b})$.

Perceived health. As described in Chapter 4, alcohol use, eating habits, and exercise all have implications for physical health. Too much alcohol, poorly chosen food, and a lack of exercise have detrimental effects, whereas moderate drinking, healthy foods, and regular exercise have beneficial effects. Thus I hypothesize that those whose health behaviors are poorer than usual during times of higher stressors will have poorer perceived health at follow-up. Conversely, those whose health behaviors improved during times of higher stressors will have better perceived health at follow-up (H2c).

\section{Contributions}

This dissertation attempts to expand the examination of the relationship between naturalistically-measured reactivity and health. In this dissertation, nurses' reactivity, as measured by changes in health behavior, is used as a predictor of later health and wellbeing. This is accomplished through the use of longitudinal data and the novel statistical technique of slopes-as-predictors, which thus far in the social sciences has been primarily used in the depression literature. Through applying these methods to this sample, this dissertation attempts to make multiple contributions to the body of scientific knowledge. First, it can contribute to the understanding of the well-being of nurses, who are also an important contributor to the well-being of others. Second, it can begin to explore the relationship between immediate reactions to stressors and longer-term health, specifically 
whether or not reactivity predicts later health outcomes. Finally, it offers a look at a potential pathway for the known link between stressors and health outcomes. 
Chapter 6: Method

\section{The Oregon Nurse Retention Project}

The Oregon Nurse Retention Project (ONRP, Sinclair et al., 2009) was a collaborative effort between researchers at Portland State University and the Oregon Nurses Association (ONA) to investigate nurse retention and turnover in the state of Oregon. As a member of the ONRP team I managed the participant database and incentive information. I also participated in each phase of the study: the planning of procedures, selection of scales, focus groups and development and pretesting of survey instruments, collection of data, cleaning and programming of data, analysis, and preparation of the final ONRP report. As seen in Figure 4 and described in the previous chapter, the ONRP agenda as a whole was focused on the ways in which work experiences, individual factors, and organizational context combine to produce nurse retention and turnover outcomes. This dissertation focuses on work stressors, but uses health and well-being outcomes rather than retention and turnover, and focuses specifically on how those outcomes relate to health behavior change in reaction to stressors. Such relationships are still nested within the ONRP model, and could be viewed as another contributor to retention and turnover, particularly given the link between depression and burnout discussed in Chapter 4.

\section{Participants}

Participants were registered nurses working in the state of Oregon. They were recruited through multiple contact methods. ONA included information about the study in mailings and newsletters. Researchers attended ONA events and took sign-ups. Nurses who heard about the study from colleagues or one of these methods were 
welcome to sign up on the study website regardless of whether or not they were ONA members. Those who signed up $(\mathrm{N}=620)$ were invited to complete a baseline survey (Wave 1), which 438 nurses completed (71\%). Of those, 144 agreed to participate in a weekly online diary survey for 12 weeks. The full Wave 1 sample was then invited to complete a follow-up survey (Wave 2) approximately four months after Wave 1, and 343 nurses completed that survey (78\%). This dissertation focuses on the weekly data, and thus the nurses who participated in that portion of the study are the sample for this dissertation. At least three weeks of data are necessary to compute a valid slope for the analyses pursued here, so seven nurses who completed fewer than three weeks of data were dropped from the sample, resulting in an $\mathrm{N}$ of 137 out of the 144 nurses who participated in the weekly survey. Among those 137 participants, 92\% were female, $5.8 \%$ male, and $2.2 \%$ did not provide their gender. Participants ranged in age from 22 to 66 years and their average age was $44.2(\mathrm{SD}=10.8)$. The majority $(92 \%)$ identified their race/ethnicity as White, with an additional $2.2 \%$ of the sample of Asian descent, $2.2 \%$ of Hispanic/Latino descent, and 3.6\% of other or multiple ethnic backgrounds. Most were married (69.3\%), 13.9\% were divorced, $8.8 \%$ had never been married, and $7.3 \%$ were living with a significant other or domestic partner. Most had an Associates (26.3\%) or Bachelors (48.9\%) degree in nursing or another field (15.3\%), and an additional $5.8 \%$ had a post-graduate degree.

\section{Measures}

At Wave 1, participants completed measures that included demographic information and other measures that are included in some hypothesis tests, specifically age, sex, and neuroticism, as well as an initial measure of perceived health. Measures of 
negative events and health behaviors were completed weekly. At Wave 2, participants completed outcome measures of subjective well-being, depression, health behavior, and perceived health. Details on these measures, as well as measures of internal consistency for those scales where they are appropriate, are in each section below.

Weekly negative events. Event measures were developed by the ONRP team through key informant interviews and focus groups with nurses, as well as a review of existing work event and nursing literature, generating a list of negative events that were used in the weekly diary. A detailed description and analysis of that process and the prevalence of the negative events in the weekly diaries was published in the ONRP report (Sinclair et al., 2009).

The weekly event questions that were developed asked during how many of the shifts worked that week a nurse experienced each event over the past 7 days, ranging from 0 " 0 shifts" to 6 " 6 or more shifts." Within the ONRP framework these 21 events were generally conceptualized as either demands (14) or conflicts (7). Overall, demands tended to be more commonly encountered than conflicts (Sinclair et al., 2009). Exploratory factor analysis of these events suggested three clusters: performance constraints, conflicts with patients, and conflict with coworkers (Deese et al., 2009). This was viewed as generally consistent with the original conceptualization of these events as being either demands or conflicts. For a list of these events and the other measures from the ONRP that were used in this dissertation, see Appendix A. The negative events can be considered as one group of 21 and as separate groupings of conflicts and demands.

The count of shifts format produced, in raw frequency terms, a possible number of negative events ranging from 0 to 126 (21 events $\mathrm{x} 6$ shifts), a possible range of 
demands from 0 to 84 (14 events $\mathrm{x} 6$ shifts), and a possible range of conflicts from 0 to 42 (7 events x 6 shifts). Using those raw frequencies, average scores were created for each participant-week: one each for negative events, conflicts, and demands; thus the maximum possible score for each group would be 6 for an individual who had every event in the group happen on 6 or more shifts that week, and the minimum possible score would be 0 for someone who had no events from the group happen that week. As these are checklist-type measures, calculating internal consistency was inappropriate for these scores.

A sample negative event is "I had a patient whose health unexpectedly declined." Some of the events selected for the measure seemed likely to be relatively common across all participants (e.g., "I watched a patient suffer"), whereas others seemed likely to be relatively uncommon for most participants, but potentially important for those who experienced them (e.g., "I was sexually harassed"). Ultimately, the sexual harassment item was indeed the least common, and the most common was equipment problems (Sinclair et al., 2009). As mentioned above, the ONRP team sought out extensive consultation by nurses during the development of the event list; that consultation served two major purposes: to ensure that relevant events that may have been left out of the literature to date and that might not be obvious to those outside the profession would be included, and to ensure that the events on the list are ones that are usually appraised as negative or stressful by nurses.

Health and health behavior. Nurses were asked seven weekly questions about their health behavior, including diet, exercise, and use of alcohol. Alcohol items were derived from the NIAAA quantity/frequency (QF) measure (Armor, Polich, \& Stambul, 
1978) and included "In the past 7 days, on how many days did you drink alcohol?" QF measures are commonly used in alcohol research, and center on measures of how often an individual drinks and how much they typically drink at those times (Dawson, 2003). Participants who reported no alcohol consumption whatsoever during the 12 weeks of the study were treated as non-drinkers and excluded from analyses related to drinking. Diet and exercise items were based on the same structure of how often/how much, including items such as "On how many days in a typical week did you engage in aerobic or physical activity, such as walking, jogging, or cycling?" See Appendix A for the full list of alcohol, health, and diet items. Each health behavior question is effectively a singleitem measure, therefore internal consistency measures were not calculated.

Perceived health was measured at Wave 1 and Wave 2 with an item based on Chipperfield (1993), “Compared to other people your age, how would you rate your health?" which was rated on a scale from 1 "much better" to 5 "much below average." For analysis purposes, I reversed the coding so that higher scores correspond to better perceived health, thus in the context of this dissertation 5 corresponds to "much better" and 1 to "much below average." Similar perceived health items have been shown to be correlated with more symptom-based measures of physical health (Shmueli, 1999).

Psychological well-being. Participants completed two scales at Wave 2 that measure mental health and related outcomes. They completed the 9-item version of the Center for Epidemiological Studies Depression scale (CES-D; Santor \& Coyne, 1997), which measures depression with items such as "In the past 30 days, how much have you felt depressed?" which are rated from 0 "rarely or none of the time" to 3 "all of the time." Higher scores therefore indicate a greater level of depression. The CES-D is widely used 
and has been validated for a variety of populations, including both inpatient and outpatient samples (Radloff, 1977). Possible scores range from 0 to 27; the internal consistency coefficient was $\alpha=.86$.

Nurses also completed the 5-item Satisfaction With Life Scale (SWLS; Diener, Emmons, Larsen, \& Griffin, 1985), a measure of SWB which contains items such as "In most ways my life is close to my ideal" rated on a scale from 1 "strongly disagree" to 7 "strongly agree" to assess subjective well-being. Higher scores therefore indicate greater life satisfaction. The scale has been validated for use with a wide range of populations, and its items have been found to represent a unified factor (Pavot \& Diener, 1993). Possible scores range from 5 to 35; the internal consistency coefficient was $\alpha=.92$ for this sample.

Control variables. Nurses also provided demographic information at Wave 1, specifically age and sex, as well as 5 items assessing neuroticism which were drawn from the International Personality Item Pool (IPIP; Goldberg, 1999) and the Core SelfEvaluations Scale (CSES; Judge, Erez, Bono, \& Thoresen, 2003). A typical neuroticism item was "I have frequent mood swings," and items were rated on a scale from 1 "strongly disagree" to 5 "strongly agree." Higher scores indicate greater levels of neuroticism and lower scores indicate greater levels of emotional stability. The range of possible scores is therefore from 5 to 25 ; internal consistency coefficient for the neuroticism items was $\alpha=.69$. This coefficient is lower than that for other measures used here; however it is only slightly lower than the coefficient for the original CSES measure and similar to that for other brief measures of neuroticism (Gosling, Rentfrow, \& Swann, 2003; Judge et al., 2003). Also, as seen in the next chapter, neuroticism did 
demonstrate many of the relationships to other variables that would be expected based on existing research, and therefore the coefficient for this sample does not seem to be a major concern.

I used age, sex, and neuroticism as control variables in the regression models generated to test Hypothesis 2 primarily because of their previously demonstrated relationships to one or more of the variables of interest. Specifically, sex was included as a control because of the predominantly $(92 \%)$ female nature of this sample and because risk of depression has been found to be greater among women than men (Kendler, Kuhn, \& Prescott, 2004). Neuroticism has also been previously associated with reactivity, as discussed in Chapter 3, and is a known predictor of psychological distress (Bolger \& Schilling, 1991). Age and neuroticism both have documented relationships to subjective well-being; greater SWB is associated with older age and greater levels of neuroticism are associated with lower SWB (Keyes, Shmotkin, \& Ryff, 2002; Lucas, 2008). Previous research on perceived health has also found associations with both age and neuroticism; perceived health has been found to be better among older individuals and poorer among those with greater levels of neuroticism (Cockerham, Sharp, \& Wilcox, 1983; Watson \& Pennebaker, 1989).

Reactivity. The measures of reactivity were generated within the data analysis procedure, specifically through the testing of Hypothesis 1; this is also the first step of the slopes-as-predictors procedure. Individual-level slopes were extracted from each eventhealth behavior combination (average weekly negative events/conflicts/demands and eating/exercise days/exercise minutes/drinking days/number of drinks, up to a total of 15 possible slopes) that had significant variance under Hypothesis 1, as described in more 
detail in the analysis section below. These slopes represent the average relationship between the event measure and health behavior over the course of the weekly study; for instance if an individual tended to drink on more days than usual during weeks in which she encountered more negative events than usual, her slope for Negative Events-Drinking Days would be positive, and if she tended to drink on fewer days than usual in those weeks, her slope would be negative. Thus a higher absolute value of the slope indicates greater reactivity, whereas slope values closer to zero indicate less reactivity. As a multilevel within-person analysis, an individual's level of reactivity is only linked to her own average behaviors, thus an individual who typically drinks frequently could potentially be either more or less reactive than one who drinks infrequently.

\section{Procedures}

Given that the ONRP targeted a large number of people from a population, nurses, that tends to be very busy during their workweek, we opted for a web-based survey both for the convenience to the nurses and the practicality of the sample size. We also chose to use a week as our time frame, given the demands on nurses' time during their average workday, the mixture of potentially common and uncommon work events we were asking about, and the variables of interest. Although some items included in the broader ONRP survey, like physical health symptoms (e.g., a headache), might fluctuate daily, many concepts of interest such as burnout and engagement seemed unlikely to change day to day. The workweek also seems to be a reasonable amount of time for retrospection on health behavior such as drinking (Heeb \& Gmel, 2005), and hangs together well conceptually as a unit of time when considering how one's work life has 
been lately. As noted in Chapter 4, other studies of work have used a weekly time frame for their diaries (e.g., Totterdell et al., 2006).

Participants provided demographic information when signing up for the study on the study website. The participants who signed up were then invited to take the Wave 1 survey. Approximately $71 \%$ of those invited completed Wave 1 . At the beginning of the Wave 1 survey, they were given and indicated their agreement with the informed consent document for the study. Those who completed the Wave 1 survey online were asked at the end of the survey if they would be willing to participate in the weekly diary portion of the study. Those who agreed were invited to participate with an email, and each weekly diary remained open for a 48-hour period to allow nurses with busy schedules enough time to complete it. If nurses missed the first two weeks of the weekly survey, they were sent a reminder email. All participants who had completed the Wave 1 survey were invited to complete the online Wave 2 survey approximately four months later. Of those who had completed the Wave 1 survey, $78 \%$ completed the Wave 2 survey also. Incentives were $\$ 20$ for the Wave 1 survey, $\$ 5$ per weekly survey, and $\$ 10$ for the Wave 2 survey.

\section{Analysis}

Data preparation. A number of data preparation steps were necessary prior to hypothesis testing. First, patterns of missing data were examined (Hofer \& Hoffman, 2007; Little \& Rubin, 2002). In the case of a repeated measures analysis, there is often sufficient data available from a participant's completed measures to justify the assumption that the incomplete data is missing at random (MAR) and therefore the partial data that is present from an individual may be retained for analysis (Hox, 2010). The 
assumption of MAR allows for differences between participants who provided complete data and those who did not, but requires that the missing data itself not be the difference between participants who provided data and those who did not (Hox, 2010; Little \& Rubin, 2002). For instance, if only the heaviest drinkers dropped out of the study after a few weeks, thus declining to provide further data on drinking, or only the heaviest drinkers did not respond to the drinking questions, the assumption of MAR would be suspect for this dissertation, in which drinking is a key variable of interest. On the other hand, if all of the dropouts were men, that would not threaten the assumption of MAR because gender was not a variable of interest.

Given the demands of a nursing schedule and the 48-hour weekly window available to complete the survey, it is perhaps not surprising that over the course of a 12week survey, most nurses missed one or more weeks of data. Average number of completed weeks for the 137 nurses retained for these analyses was $9.8(\mathrm{SD}=2.1)$, with a range from 3 to 12 . Number of weeks completed did not prove to be statistically related to any of the outcome variables of interest, and therefore all cases in this group were retained. The statistical software used for the Hypothesis 1 analyses, Hierarchical Linear Modeling (HLM; Raudenbush, Bryk, Cheong, Congdon, \& du Toit, 2011) is designed to handle incomplete data. Item-by-item nonresponse was low for the weekly data, with less than $3 \%$ of completed weeks missing one or more variables used in this dissertation, and item-by-item rates of missing data were also less than $3 \%$ for the variables of interest at Wave 1 and Wave 2. Of the 137 weekly participants chosen for this sample, nine (7\%) did not complete the Wave 2 survey. Their data was therefore available for tests of 
Hypothesis 1 but not Hypothesis 2. Item-by-item missing data did not relate statistically to any variables of interest, thus all cases were retained for analysis.

I also examined the distributions and frequencies of all variables of interest, allowing detection of any errors that may have been missed during data cleaning, as well as familiarizing myself with the patterns of the data. Through this examination I also confirmed that sufficient variability exists for these analyses. If, for example, all nurses had given themselves the highest perceived health rating at the time of Wave 2, analyses including that variable would be fruitless. Outliers can be detected both through this visual examination of the data, and through the use of Mahalanobis distance, which is a way of quantifying the effect any particular data point has on the regression it would be included in (Tabachnick \& Fidell, 2007). Thus a large Mahalanobis distance would be indicative of an individual whose data might have a disproportionately large influence on results. One participant proved to have consistently consumed a considerably larger number of drinks than others in the sample (mean weekly Mahalanobis distance $=87.15$ ), and was therefore removed from analyses that included number of drinks but retained for analyses of all other variables. Distributions of key variables were also examined for normality, and no transformations or non-linear models were deemed necessary.

In this fashion, I examined not only the key variables of interest, but also the control variables of age and neuroticism. Because of the gender-imbalanced nature of the nursing profession and thus this sample, I tested for gender differences in key areas where they might be expected based on prior research, such as alcohol consumption, which has been found to be higher and more frequent among men (Wilsnack, Vogeltanz, Wilsnack, \& Harris, 2000) and reporting of work stressors, which has also been found to 
be higher among men (Almeida, 2005). Both of these gender differences were also found in this sample (see Chapter 7), but because gender could be included in the Hypothesis 2 regression analyses as a covariate, I opted to retain the men in the sample. My more frequent use of feminine than masculine pronouns in this dissertation reflects the largely female sample, but is not intended to exclude the male participants in any way.

After examining the data, additional preparation was necessary prior to testing Hypothesis 1. The within-person longitudinal nature of the data means that the assumption of independence of data is not fulfilled because repeated measures are coming from the same individual. Therefore multilevel modeling was appropriate for the testing of this hypothesis. HLM (Raudenbush et al., 2011) is designed to deal with unbalanced data, and generated slopes and intercepts for the testing of Hypothesis 1 even if individuals have different numbers of valid data points. To compare individuals' responses in any given week to their overall typical patterns of behavior, centering of the data was necessary (Enders \& Tofighi, 2007). Specifically, for all variables entered into the models tested under Hypothesis 1, the question of interest is not, for instance, how individuals' drinking and stressors related to other individuals' drinking and stressors, but rather how individuals' drinking and stressors varied across weeks in comparison to their own usual patterns. In other words, the question was not whether Nurse A drank more than Nurse B, but whether Nurse A drank more than she usually does in a week when she encountered more stressors than she usually does, whereas Nurse B's drinking in a week with more stressors than usual might not increase relative to Nurse B's usual amount. Person-centering the weekly variables eases such comparison by changing the values without changing the scale (Enders \& Tofighi, 2007). In this process, each individual's 
mean levels of weekly variables are calculated, then that mean is subtracted from each week's values. This centering was specified as part of the analysis setup and therefore completed automatically by the HLM program.

Multilevel modeling allows researchers to account for the dependency that is inherent within data that comes from repeated measures with the same individuals (i.e., weekly diaries), and moreover it provides the ability to test for weekly change as measured on the individual rather than aggregate level, as discussed above (Snijders \& Bosker, 1999; Walls, Jung, \& Schwartz, 2006). For example, simply averaging the sample's drinking and stress would not only inappropriately ignore the dependency in the data, it would also possibly lead to misleading results. Multilevel modeling in which individuals are at Level 2 and their person-centered weekly questionnaire responses are at Level 1 allows the detection of deviations from individual norms, which would not be visible in traditional regression.

In addition to specifying centering within HLM, it was important to include contrasts between weeks of the study to test whether patterns of response differed through the study. If, for instance, nurses felt less comfortable with the weekly survey during the beginning of the study, they might tend to under-report their drinking at the beginning of the study but report it more honestly later on; alternately, if a particular week of the study contained a major event such as a holiday weekend when hospitals might be busier than usual, stressors could be different in one particular week of the study as compared to others. Therefore I created eleven dummy variables for each of weeks 1 through 5 and 7 through 12, using week 6 (just prior to the study midpoint) as a comparison (Hox, 2010). Comparing other weeks to a central week allowed me to 
determine if there are systematic differences between weeks of the study. These dummy variables were modeled as fixed effects to test for potential patterns of systematic variation through the weekly diaries that might necessitate the inclusion of such contrasts in the hypothesis testing models described below. Some differences with an unclear pattern were present, therefore to be conservative in the analyses the dummy variables were retained for the tests of Hypothesis 1.

Slopes-as-predictors. The slopes-as-predictors analysis procedure has two major steps (L. H. Cohen et al., 2005; Mohr et al., 2013). First the weekly slopes are calculated, as in a typical within-person multilevel analysis. This was accomplished by using HLM 7.0 (Raudenbush et al., 2011) to calculate multilevel models in which observations are nested within persons, with person-centered weekly negative events predicting each weekly health behavior or other outcome. For example, negative events predicting drinking days would be one model, negative events predicting exercise days another. In these models, events and behaviors were modeled as random, which allowed significance testing of the variance between individuals. These models served as the tests for Hypothesis 1 . I examined the unstandardized coefficients, $R^{2}$, and squared semi-partial correlations for each resulting multilevel model. Significant variance components in each model were predicted under Hypothesis 1a-1e. Thus, Hypothesis 1 was tested with these multilevel models, and Hypothesis 2 was tested with the second step of the slopes-aspredictors described below, essentially a series of post-hoc tests to the multilevel models used in Hypothesis 1. For combinations of the three events scales and five health behaviors for which there was no significant variance between individuals, it was not appropriate to continue with the slopes-as-predictors procedure, since there is no reason 
to believe that multiple distinct patterns of individual variability might be present (Mohr et al., 2013).

To illustrate this, I will use as an example Hypothesis 1c (there will be significant variance between persons in the weekly relationship between nurses' negative events and their eating behavior), which was tested via the following regression equation:

Level 1: AteHealthy $y_{i t}=b_{0 i}+b_{1 i}\left(N_{t}\right)+e_{i t}$

Level 2: $\mathrm{b}_{0 \mathrm{i}}=\gamma_{00}+\mathrm{u}_{0 \mathrm{i}}$

$$
\mathrm{b}_{1 \mathrm{i}}=\gamma_{10}+\mathrm{u}_{1 \mathrm{i}}
$$

In this equation, AteHealthy $y_{i t}$ is the endorsement of the "I ate healthy foods" questionnaire item for person $\mathrm{i}$ in week $\mathrm{t}, \mathrm{b}_{0 \mathrm{i}}$ is the predicted value for that item when negative events are at the person-centered mean during that week, $b_{1 \mathrm{i}}$ is the partial withinperson regression coefficient for negative events (NE, the predictor) for person $\mathrm{i}$, and $\mathrm{e}_{\mathrm{it}}$ is the random residual component. This equation would be repeated four more times, substituting each health behavior. Level 2 equations were the same for each analysis, since no person-level predictors (e.g., age) were included in these tests. In addition to testing Hypothesis 1c, that there will be significant variance in the relationships between negative events and eating behavior, this model also yielded the individual-level slope for NE-AteHealthy, which would enter into the subsequent regression models used to test Hypothesis 2 if that test of Hypothesis 1c passed. Contrasts based on the week of the study, as described earlier in this chapter, were also included in the analysis but for the sake of clarity have been omitted from the equation displayed above.

For the event scale-behavior combinations for which it was appropriate to continue with the slopes-as-predictors analysis, individual events-drinking, events- 
exercise, etc. empirical Bayes slopes were extracted from the residuals files produced in the running of these models (Mohr et al., 2013). In the second major step of the slopesas-predictors procedure, these individual slopes then became person-level variables that were used as predictors in subsequent regression models, in which the weekly eventbehavior slopes predicted outcomes at Wave 2 to test Hypothesis 2. 
Chapter 7: Results

\section{Stressors and Health Behaviors}

Descriptive information. Before proceeding to the tests of Hypothesis 1, I examined the descriptive statistics for the sample, which were reported in Chapter 6, as well as for the variables and covariates of interest. Among the health behavior variables, endorsement of the healthy eating item ranged from 1 to 5 , with a mean of $3.65(\mathrm{SD}=$ 1.03), which would fall between "sometimes" and "often" on the response scale; number of exercise days ranged from 0 to 7 , with a mean of $2.41(\mathrm{SD}=1.99)$; the categories of exercise minutes ranged from "0-20 minutes" to " $70+$ minutes," with the mean and median both falling into the "30 minutes" category. Number of drinking days ranged from 0 to 7 , with a mean of $2.04(\mathrm{SD}=2.03)$; and number of drinks ranged from 0 to 5 , with a mean of $1.01(\mathrm{SD}=.93)$. Calculating negative event scores on a 0 to 6 scale as described in Chapter 6 yielded negative event scores that ranged from 0 to 2.57 with a mean of $.30(\mathrm{SD}=.36)$, which would correspond to the average nurse in the average week encountering 6.25 negative events. Those six events could, for example, indicate

encountering three different events on two shifts each, or six different events in the same single shift, or any other combination of shifts and events that totals six. Demand scores ranged from 0 to 2.79 with a mean of $.37(\mathrm{SD}=.46)$, which would correspond to the average nurse in the average week encountering 5.11 demands. Finally, conflict scores ranged from 0 to 2.14 with a mean of $.16(\mathrm{SD}=.27)$, which would correspond to the average nurse in the average week encountering 1.14 conflicts.

I also examined the correlations of the components of these slopes with the covariates and outcomes planned for use in the tests of Hypothesis 2, as displayed in 
Table 2. Among the slope components, healthy eating and the exercise variables were significantly positively correlated with one another, which would be consistent with an attempt to maintain good health practices. The two exercise measures were significantly positively correlated, as were the two drinking measures, as would be expected for different measures of the same behavior. Similarly, conflicts and demands were significantly positively correlated. Exercise minutes were also significantly positively correlated with number of drinks and all three negative event measures, which suggests that those who exercised for longer periods of time on average also were both encountering more stressors and drinking more; however number of drinks itself did not correlate significantly to the negative event measures.

There were also several significant correlations between slope components and outcomes. Healthy eating was significantly positively correlated with satisfaction with life and perceived health, and significantly negatively correlated with depression scores, suggesting an association between healthy eating and better outcomes for each of those variables at Wave 2. Exercise days were also significantly positively correlated with SWL, and both exercise days and minutes were significantly positively correlated with perceived health. Significant positive correlations also emerged between depression scores and the negative events and demands measures, with a similar positive correlation to conflicts that did not reach significance $(p<.10)$, suggesting an overall association between negative work events and depression symptoms. Similarly, there was a significant negative correlation between demands and satisfaction with life. Among demographic factors, age was significantly positively correlated with drinking days, and respondents differed by sex on the three negative event measures. Specifically, males 
tended to report more demands $(.73$ vs $.35 ; F(1,132)=8.79, p<.01)$, conflicts $(.33$ vs. .15; $F(1,132)=5.77, p<.05)$, and overall negative events $(.60$ vs. $.28 ; F(1,132)=9.20, p<.01)$ than females. As described previously, I had already planned to include sex as a control variable in the Hypothesis 2 regression models because of the extremely unbalanced $\mathrm{N}$ of males and females; these differences supported that decision.

Hypothesis 1 tests. There will be significant variance between persons in the weekly relationship between nurses' negative events and their health behaviors.

This hypothesis was tested for a total of five measures that captured three types of health behaviors: (1a) days of exercise, (1b) minutes per day of exercise, (1c) endorsement of the "I ate healthy foods" item, (1d) number of drinking days, and (1e) number of drinks. Specifically, this was a multilevel analysis with individuals at Level 2 and the person-centered negative event variable being tested and the person-centered health behavior variable being tested at Level 1. The Level 1 variables were modeled as random, thus allowing the test of variance. For the drinking variables, a Poisson sampling model was used, since drinking variables are counts that also tend to have appropriately shaped distributions for that model (e.g., skewed). The areas of focus in these results were the relationship between negative events and exercise for H1a and $\mathrm{H} 1 \mathrm{~b}$, the relationship between negative events and eating healthy foods for $\mathrm{H} 1 \mathrm{c}$, and the relationship between negative events and drinking for $\mathrm{H} 1 \mathrm{~d}$ and H1e. Overall, this hypothesis tested for variability in reactivity to work stress as demonstrated by changes in health behaviors. That was operationalized as individual combinations of each of the three stressor measures (overall negative events, demands, and conflicts) with each of the five health behaviors (number of drinks, drinking days, exercise minutes, exercise days, 
and healthy eating), resulting in 15 separate multilevel models, each of which was tested using HLM. The results of those tests are displayed in Table 3.

The models revealed significant average within-person associations between number of drinks and overall negative events and conflicts, as well as between conflicts and drinking days. In each case, the results indicate a tendency for nurses in this sample to drink less during weeks of greater stressors. The model that included number of drinks and demands was also consistent with this pattern, but only approached significance $(p<.10)$. For the model including conflicts and drinking days, however, a significant variance component was also present, indicating that despite the existence of an overall pattern, there was significant variability among nurses' relationships between conflicts and drinking days. In other words, although the general tendency reflected by the average within-person association was for most nurses to drink less than usual in weeks when they encountered more conflicts than usual, there was a significant amount of variation among nurses in the extent to which that happened, and for some nurses, the reverse pattern may have been present. This finding of significant variability constitutes partial support for Hypothesis $1 \mathrm{~d}$.

Each set of results for Hypothesis 1 were considered separately. For each negative event-health behavior relationship, if significant variance was found, it was appropriate to continue on to the testing of Hypothesis 2 for that relationship. If no significant variance was found, there is no reason to believe that distinct individual patterns of reactivity exist for that variable within this sample, and thus testing of Hypothesis 2 was not justified. Ultimately, out of fifteen event-behavior combinations (3 event scores X 5 health behaviors) five slopes demonstrated significant variance, as 
displayed (in bold) in Table 3. Specifically, H1a, examining variability in the relationship between negative events and days of exercise, was supported for conflicts, demands, and overall negative events. H1b, examining variability in the relationship between negative events and minutes of exercise, was supported only for overall negative events, although the variance component for conflicts did approach $(p<.10)$ significance. H1c, for healthy eating, was not supported, but did approach significance $(p<.10)$ for demands. Thus for healthy eating, neither significant variability nor significant withinperson associations were found. H1d, for drinking days, was supported only in the case of conflicts, as discussed above, and H1e, for number of drinks, was not supported. In the case of number of drinks, the significant average within-person associations suggest that there is a general overall pattern present in this sample rather than variability. In summary, H1 was supported for five of the fifteen slopes, indicating it was appropriate to continue to tests of Hypothesis 2 for the following slopes: Negative Events-Exercise Days, Negative Events-Exercise Minutes, Conflicts-Exercise Days, Conflicts-Drinking Days, and Demands-Exercise Days.

\section{Reactivity Slopes and Outcomes}

Descriptive Information. Descriptive statistics for the slopes calculated during the testing of Hypothesis 1 and for the covariates and outcomes included in the regression models used to test Hypothesis 2 are reported in Table 4. As described in Chapter 6, the sample was $92.0 \%$ female and 5.8\% male, with an additional 2.2\% unknown (missing). Among other covariates, the average neuroticism score was 12.78 of a possible 25 , and average age was 44.22 years. Among the outcomes examined in this dissertation, nurses rated their perceived health at an average of 3.75 , which would correspond to a score 
slightly lower than "above average," average CES-D score was 6.53 of a possible 27, and average satisfaction with life was 25.24 of a possible 35. Most nurses, therefore, were relatively satisfied with their lives, were experiencing few depression symptoms, and rated their perceived health relatively well.

All five slopes that were eligible for examination under Hypothesis 2 included both negative and positive values, indicating that, as expected, for some nurses the health behavior in question tended to increase when the stressors in question increased, whereas for other nurses the health behavior in question tended to decrease when the stressors increased. In fact in all five cases, the mean slope between persons was negative. Specifically, $76.5 \%$ of participants had a negative slope for Conflicts-Drinking Days, 65.0\% for Conflicts-Exercise Days, $80.3 \%$ for Demands-Exercise Days, $77.4 \%$ for Negative Events-Exercise Days, and 63.5\% for Negative Events-Exercise Minutes. This suggests that on average, nurses in this sample may have been more likely to decrease rather than increase the health behaviors in question when their stressors increased. However, as discussed above, this only took the form of a significant average withinperson association for Conflicts-Drinking Days; thus the significant variability found for these five slopes should be kept in mind when examining the means. Although the average slope was negative, as the minimum and maximum values reveal there was a great deal of individual variation in the nature of the relationship between the stressors and health behaviors. Some nurses, whose slopes are closer to zero, changed their behaviors very little in weeks when their stressors varied from their mean, whereas others, whose slopes are farther from zero in either direction, changed their behaviors more when their stressors varied from their mean. 
The overall distributions of these five slopes are displayed in Figures 5a-5e to allow for a visual examination of the prevalence of the various slope patterns within the sample. As is visible in these figures, the slopes as a whole tended to be relatively normally distributed. Thus there was no need to transform the slopes prior to conducting further analysis.

Intercorrelations. I examined the correlations among the slopes, covariates, and outcomes, which are displayed in Table 5 with significant correlations of slopes with outcomes in bold. Nurses' Conflicts-Exercise Days slopes were significantly positively correlated with their SWLS scores, as seen in Table 5, indicating that the more nurses' exercise days increased in weeks when their conflicts increased, the higher their scores on the SWLS tended to be at Wave 2. Both the Conflicts-Exercise Days slope and the Negative Events-Exercise Days slope were significantly positively correlated with perceived health at Wave 2, indicating that the more nurses' exercise days increased in weeks when their conflicts or negative events increased, the better their perceived health at Wave 2 tended to be. The correlations among covariates and outcomes were overall quite conceptually consistent: perceived health was significantly positively correlated with satisfaction with life and age, and significantly negatively correlated with depression scores and neuroticism; satisfaction with life was significantly negatively correlated with neuroticism and depression scores; and depression scores and neuroticism were significantly positively correlated. Intercorrelations among the slopes themselves tend to correspond to the commonality amongst the event measures and the health behaviors, for instance, slopes involving exercise (minutes or days) tend to be correlated with one another. 
Hypothesis 2 tests. Nurses who show greater weekly reactivity in the form of increased drinking, decreased exercise, or poorer eating habits will have poorer health and well-being outcomes at follow-up; conversely, nurses who show greater weekly reactivity in the form of increased exercise or better eating habits will have better health and well-being outcomes at follow-up.

The five individual-level slopes extracted from the analyses conducted for $\mathrm{H} 1$ were entered into separate regression models, each in turn, as reactivity slopes for each participant (Negative Events-Exercise Days, Negative Events-Exercise Minutes, Conflicts-Exercise Days, Conflicts-Drinking Days, and Demands-Exercise Days). Specifically, the three health and well-being outcomes were then regressed on those five slopes, resulting in a total of 15 combinations of slopes and outcomes.

The Wave 2 outcomes of interest were depression scores (2a), satisfaction with life (2b), and perceived health (2c). Because of their previously discussed known associations with key outcomes and the previously discussed characteristics of this sample, three covariates (age, neuroticism, and sex) were also entered into the models. This resulted in five models for each of the three hypotheses, one for each measure of reactivity for each outcome. Of particular interest here were the relationships between the assorted measures of reactivity and depression scores for $\mathrm{H} 2 \mathrm{a}$, between reactivity and life satisfaction for $\mathrm{H} 2 \mathrm{~b}$, and between reactivity and perceived health for $\mathrm{H} 2 \mathrm{c}$. The regression models are displayed in Tables 6,7, and 8. The squared semi-partial correlation coefficient displayed in those tables for each variable represents that variable's individual contribution to the change in $R^{2}$ for the model. 
H2a: Depression scores. The regression models to test the hypotheses pertaining to depression scores included a negative event-health behavior slope (Negative EventsExercise Days, Negative Events-Exercise Minutes, Conflicts-Exercise Days, ConflictsDrinking Days, and Demands-Exercise Days) and CES-D scores at follow-up, as well as age, sex, and neuroticism. All five models were significant. In two models, reactivity was a significant predictor of CES-D scores, and it approached significance in a third. Those models are presented at the start of Table 6 with the significant reactivity coefficients in bold. Neuroticism also significantly predicted CES-D scores in all five models, but no other covariates emerged as significant predictors.

Specifically, Model 1, for Demands-Exercise Days, was significant at $F(4,117)=9.55, p<.001$. Model 2, for Negative Events-Exercise Days, was also significant at $F(4,117)=9.81, p<.001$. In both cases, the significant coefficient for reactivity was negative, which is in line with the predicted pattern, specifically that more positive reactivity slopes for Demands-Exercise Days and Negative Events-Exercise Days would be associated with lower depression scores, and conversely that more negative reactivity slopes for exercise would be associated with higher depression scores. To illustrate for the specific example of Model 1, the coefficient for reactivity (the Demands-Exercise Days slope) was -2.15 , which in the context of the regression equation would indicate that for every one-unit increase in that slope, CES-D scores would go down by 2.15 points. A nurse whose Demands-Exercise Days slope was .50 , indicating a general trend towards having more exercise days than usual in a week when demands were greater than usual, would, therefore, have a depression score 1.08 points lower $(.50$ * $-2.15=-1.08)$ than a nurse whose slope was zero, indicating no relationship between 
demands and exercise days. Whereas an otherwise identical nurse whose slope was -.50 , indicating the reverse pattern of fewer exercise days in weeks of greater demands, would have a depression score 1.08 points higher $\quad(-.50 *-2.15=1.08)$ than a nurse whose slope was zero. Thus positive event-exercise slopes were associated with lower depression scores and negative event-exercise slopes were associated with higher depression scores. In other words, those who engaged in more days of exercise than usual during weeks with more demands or negative events than usual were likely to have lower depression scores than those who engaged in fewer days of exercise during such weeks. These two models thus provide partial support for $\mathrm{H} 2 \mathrm{a}$ in the specific instances of Demands-Exercise Days and Negative Events-Exercise Days.

The same pattern of reactivity and depression scores was also present in Model 3, for Negative Events-Exercise Minutes. The model was significant at $(F(4,117)=9.24$, $p<.001)$, but in that case the $p$-value for reactivity only approached significance and did not reach the standard of $p<.05$, therefore no firm evidence of the association between events-exercise slopes and depression scores was found in that model. Models 4 $(F(4,81)=6.23, p<.001)$ and $5(F(4,117)=8.92, p<.001)$ were also significant, but the reactivity slopes were $p>.10$ in each of those cases. In the case of Model 4, for ConflictsExercise Days, the reactivity coefficient is consistent in direction with the other two models for exercise days (Model 1 and Model 2), but it was not significant. Overall, support for $\mathrm{H} 2 \mathrm{a}$ was found for two of the five slopes, with a third approaching significance.

H2b: Satisfaction with life. The regression models to test the hypotheses pertaining to life satisfaction included a negative event-health behavior slope (Negative 
Events-Exercise Days, Negative Events-Exercise Minutes, Conflicts-Exercise Days, Conflicts-Drinking Days, and Demands-Exercise Days) and SWLS scores at follow-up, as well as sex, age, and neuroticism. All five models were significant, and reactivity was a significant predictor of SWL in one model, which is presented at the start of Table 7 with the significant reactivity coefficient in bold. Neuroticism was also significantly negatively associated with satisfaction with life in all models, but no other covariates emerged as significant predictors.

Model 1, for Conflicts-Exercise Days, was significant at $F(4,117)=6.37, p<.001$. Reactivity was a significant predictor of SWL within that model, and the coefficient for reactivity was positive. This indicates that more positive slopes were associated with higher SWLS scores, and conversely that more negative slopes were associated with lower SWLS scores. Specifically, each one-unit increase in the Conflicts-Exercise Days slope would be associated with an increase of 2.16 points in a nurse's SWLS score, and each one-unit decrease would be associated with a decrease of 2.16 on that score. In other words, a nurse whose days of exercise increased during weeks of greater conflicts would have a higher satisfaction with life score than one whose days of exercise decreased during weeks of greater conflicts. This model provides partial support for $\mathrm{H} 2 \mathrm{~b}$.

The other four models were also significant; however reactivity slopes did not emerge as significant predictors in any of them. Model 2 was significant at $F(4,81)=5.47$, $p=.001$, Model 3 was significant at $(F(4,117)=5.26, p=.001)$, Model 4 was significant at $(F(4,117)=5.51, p<.001)$, and Model 5 was significant at $(F(4,117)=5.27, p=.001)$. 
Overall, support for $\mathrm{H} 2 \mathrm{~b}$ was found for one of the five slopes, specifically ConflictsExercise Days.

H2c: Perceived health. The regression models to test the hypotheses pertaining to perceived health included a negative event-health behavior slope (Negative EventsExercise Days, Negative Events-Exercise Minutes, Conflicts-Exercise Days, ConflictsDrinking Days, and Demands-Exercise Days) and perceived health at follow-up, as well as sex, age, and neuroticism. Reactivity was a significant predictor in three models, and approached significance in a fourth. Those models are presented at the start of Table 8 with the significant reactivity coefficients in bold. Age and neuroticism were also significant predictors of perceived health in the majority of models; specifically, increased age was associated with higher perceived health and neuroticism was associated with lower perceived health.

As seen in Table 8, Model 1, for Conflicts-Drinking Days, was significant at $F(4,81)=3.17, p=.02$. The coefficient was positive, indicating that the relationship between reactivity and perceived health is opposite to the predicted direction, specifically that more positive slopes were associated with higher scores for perceived health (an increase of 2.03 for every one-unit increase in slope) and more negative slopes were conversely associated with lower scores for perceived health. Thus reactivity to weeks with greater conflicts in the form of increased drinking days was associated with better perceived health, and reactivity in the form of decreased drinking days in such weeks was associated with poorer perceived health. This does not support H2c.

In contrast, Models 2 and 3 offer evidence consistent with the expectations laid out under H2c. Model 2 was significant at $F(4,117)=7.03, p<.001$, and Model 3 was 
significant at $F(4,117)=5.16, p=.001$. In those models, Conflicts-Exercise Days and Negative Events-Exercise Days were each significant predictors of perceived health, and the direction of those relationships is in line with what was predicted; specifically reactivity in the form of increased exercise is associated with better perceived health, and reactivity in the form of decreased exercise is associated with poorer perceived health. The reactivity slope for Demands-Exercise Days also follows the same pattern in Model 4, which was significant at $F(4,117)=4.66, p=.002$, but the coefficient for the reactivity slope in that model only approached significance. Models 2 and 3 provide partial support for H2c, and Model 4 is consistent with their pattern but fell short of being significant $(p<.10)$

Model 5, for Negative Events-Exercise Minutes, was significant at $F(4,117)=3.87$, $p=.005$, but the reactivity slope coefficient was not significant in that model. Overall, support for H2c was found for two of the five slopes (Conflicts-Exercise Days and Negative Events-Exercise Days) with a third demonstrating the same pattern approaching significance (Demands-Exercise Days), and evidence contradicting H2c was found for one of the five slopes (Conflicts-Drinking Days).

The perceived health measure was administered at Wave 1 as well as Wave 2, thus offering the opportunity to determine whether or not reactivity slopes were associated with changes in perceived health during the period of the study. Therefore I conducted a second set of models for perceived health in which nurses' Wave 1 perceived health score was also included as a control variable. However, the Wave 1 and Wave 2 health scores were significantly and quite highly correlated at .78, and thus inclusion of that score simply resulted in five models in which the Wave 1 level was the only 
significant predictor. Therefore there is no evidence of an association between reactivity slopes and changes in perceived health during the period of the study.

Overall, for H2a (CES-D), two slopes (Negative Events-Exercise Days and Demands-Exercise Days) were significant predictors in the expected direction, with reactivity in the form of increased exercise days associated with lower depression scores at Wave 2. The same pattern for Negative Events-Exercise Minutes also approached significance. For H2b (SWLS), one slope (Conflicts-Exercise Days) was a significant predictor in the expected direction, with reactivity in the form of increased exercise days associated with greater satisfaction with life scores at Wave 2. Finally, for H2c (perceived health), two slopes (Negative Events-Exercise Days and Conflicts-Exercise Days) were significant predictors in the expected direction, with reactivity in the form of increased exercise days associated with better perceived health at Wave 2. The same pattern for Demands-Exercise Days also approached significance. However the significant relationship between Conflicts-Drinking Days and perceived health was inconsistent with the predictions of $\mathrm{H} 2 \mathrm{c}$, specifically that reactivity in the form of increased drinking days was associated with better perceived health at Wave 2. Thus there was partial support for $\mathrm{H} 2 \mathrm{a}, \mathrm{H} 2 \mathrm{~b}$, and $\mathrm{H} 2 \mathrm{c}$. 


\section{Chapter 8: Discussion}

The purpose of this dissertation was to conduct an exploration of the link between immediate effects of stressors and later health and well-being. More specifically, I examined behavioral reactivity in the form of week-to-week changes in health behaviors that covaried with work stressors; reactivity which, in turn, predicted later life satisfaction, depression scores, and perceived health. The threefold purpose of this inquiry was to explore the linkages between immediate and longer-term effects of stressors, thereby attempt to illuminate one potential pathway through which the longerterm effects of stressors may take place, and to extend understanding of the well-being of nurses.

Although both the immediate and longer-term effects of stressors have been wellstudied, the link between those two timeframes has not. Moreover, aside from the direct physiological effects of stressors such as allostatic load, the pathways through which stressors cause longer-term outcomes remain largely unexplored. It seems possible, particularly in the case of health behavior reactivity, that those immediate reactions to stressors may in fact form part of the pathway through which the later effects occur. To begin to explore this possibility, I employed the statistical technique of slopes-aspredictors to derive a naturalistic measure of reactivity (a slope) from the variability in nurses' weekly patterns of work stressors and health behaviors, which was then used as a predictor of later outcomes.

The first hypothesis tested was that varying patterns of stressor-health behavior slopes (e.g., reactivity) would exist within the sample. As described in full in the previous chapter, partial support for that hypothesis was demonstrated, with five of the 
fifteen possible stressor-health behavior slopes demonstrating significant variability between persons. The second hypothesis tested was that those variable patterns of reactivity could predict later satisfaction with life, depression scores, and perceived health. That hypothesis was also partially supported, as described in the previous chapter, with two of the five slopes demonstrating the predicted relationship with depression scores, one slope demonstrating the predicted relationship with satisfaction with life, and two slopes demonstrating the predicted relationship with perceived health. Additionally, one slope demonstrated the opposite of the predicted relationship with perceived health, and other slopes approached significance as predictors of outcomes. In this discussion chapter, I will examine possible explanations for and implications of my results, ultimately using those results as a foundation for a discussion of possible directions for further research, the strengths and weaknesses of this dissertation, and the overall implications of my findings.

\section{Patterns of Reactivity}

My first hypothesis was designed to determine whether or not variable patterns of health behavior reactivity to work stressors existed within this sample of nurses. To do that, I examined individual variability in the relationships between the three stressor measures (conflicts, demands, and all negative events) and five health behaviors (exercise days and minutes, healthy eating, and drinking days and number of drinks). As previously described, significant variability was found for five types of reactivity (three for exercise days, and one each for exercise minutes and drinking days), thus providing partial support for Hypothesis 1. These variable patterns, where found, may reflect use of 
these three types of behaviors for coping, stressor-related failures in self-control, or simply practical limitations on time and energy.

Exercise. Nurses did tend to vary in many of their stressor-exercise relationships. Exercise days (H1a) yielded the most consistent results in support of this hypothesis, with all three models demonstrating significant variability between persons. Exercise minutes (H1b) also demonstrated partial support, with one of the three models demonstrating significant variability and a second approaching significance. The less consistent pattern for exercise minutes could imply that the number of days on which nurses exercised (2.41 days on average, as reported in Chapter 7), was more likely to change in response to stressors than the amount of exercise they did on those days (30 minutes on average). Thus stressors might lead to an extra (or missed) session of physical activity, but perhaps not a change in the type or length of that activity. Future research can probe more deeply into patterns of exercise such as types, times of day, days of the week, and work days vs. weekends, to better understand this kind of reactivity.

The patterns of results for the two exercise variables were similar. Although a majority of nurses got fewer days/minutes of exercise than usual during weeks in which they encountered more work stressors than usual, it is worth noting that, as seen in Figure 5a, many nurses did in fact get more days/minutes of exercise than usual during such weeks. This type of variability might account for why some previous studies have found no relationship between stressors and exercise (e.g., Steptoe et al., 1998), whereas others have found associations between stressors and reduced exercise (e.g., Stetson et al., 1997). In this sample, those who exercised more may have been using exercise as a coping mechanism. Those who exercised less may have decreased their exercise because 
their week was busier than usual and they lacked time or energy for exercise once their work duties were completed, or they may have experienced stressor-related self-control failure. Regardless of the specific mechanism, it seems clear that encountering more stressors changed nurses' exercise patterns in variable ways.

Eating. Eating healthy foods (H1c) demonstrated no support of this hypothesis, although in one model the variability between persons did approach significance. This failure to find variability could indicate that behavioral reactivity to work stressors in this sample did not take the form of changes in eating habits, or that stressor-related changes in eating habits such as extra snacks or emotional eating might take place at a nonconscious level that was not apparent to individuals when they thought back over their workweek in an evaluative way. There were also no significant fixed effects for eating, which is inconsistent with previous studies that demonstrated associations between stressors and additional or unhealthy snacks (Newman et al., 2007; Steptoe et al., 1998). However, unlike the other measures of health behavior (exercise days and minutes, drinking days and number of drinks), the eating measure asked for a qualitative judgment of one's behavior rather than a concrete number. It is thus possible that this measure ("I ate healthy foods") was unable to capture such reactivity if it occurred. It is possible to both eat healthy foods and engage in stressor-related changes in eating; if, for instance, a nurse ate 5 servings of vegetables every day that week, she might decide to strongly agree with that statement even if she also ate a pint of ice cream after returning home from a shift at work in which she encountered more stressors than usual. If the failure to find variability here is related to the breadth of the qualitative judgment or to the potentially non-conscious nature of changes in eating habits, a more concrete and detailed set of 
probes about eating behaviors might have produced evidence of behavioral reactivity; thus such questions should be considered for future studies.

Drinking. Most slopes for drinking were not variable between nurses. Drinking Days (H1d) demonstrated partial support of this hypothesis, with one of the three models demonstrating significant variability between persons. Number of drinks (H1e) demonstrated no support of this hypothesis. Some of the drinking slopes, however, did display significant fixed effects (one slope for drinking days and two for number of drinks; see Table 3); in all cases these effects suggest that, on average, nurses drank less when encountering more stressors. With one exception (Conflicts-Drinking Days), the variance components that accompany those stressor-drinking slopes are not significant, indicating that there was not a significant amount of variability among nurses in the magnitude and/or direction of their stressor-drinking slopes.

In the case of the one significant reactivity slope (Conflicts-Drinking Days), further examination reveals that most nurses drank on fewer days than usual during weeks in which they encountered more conflicts, but some nurses drank on more days during such weeks. In the context of this sample, in which the average nurse who drank did so on only two days per week and only consumed one drink per sitting, the small magnitude of the positive slopes suggests that the increases in drinking days that did exist in this sample were quite small. Considered alongside the significant fixed effect for Conflicts-Drinking Days, it appears that those nurses whose slopes, positive or negative, are near to zero are those who did not reduce their drinking days to the extent that those with more negative slopes did. The finding that most nurses drank less during weeks of more conflicts is consistent with previous research, such as the Carney et al. (2000) study 
in which daily negative work events increased desire to drink but not actual consumption. Nurses whose drinking days decreased during weeks of more conflicts may have simply lacked time to drink, or perhaps lacked time, energy, or enthusiasm to engage in social drinking they might normally have participated in.

Summary. Overall, for those five models in which significant variability was found between persons, patterns of reactivity were not consistent across nurses. Failure to find significant variability between persons in ten of the models could be explained by a number of possibilities. In addition to the behavior-specific possible explanations discussed above, it could be that reactivity of those types simply did not occur within this sample, perhaps because health behaviors are too habitual to exhibit much change in response to stressors. However, the variability found within a third of the models does suggest that at least some behavioral reactivity took place in this sample. Additionally, in the case of number of drinks, based on the significant fixed effects it appears that there was behavioral reactivity taking place within the sample (nurses drank less during weeks of greater stressors), but that the reactivity was too consistent in direction and magnitude across nurses to demonstrate significant between-person variability.

Because the behavioral measures were general and the stressor measures were specific to the work domain, reactivity that took place in response to nonwork stressors may also have clouded the results. For instance, a nurse who drank more than usual in response to nonwork stressors during a week in which her work stressors were at their typical level would in fact be exhibiting behavioral reactivity, but it would not be detectable by these analyses because those nonwork stressors were not measured. Workfamily conflict, another unmeasured variable in this sample, could also have led to 
behavioral reactivity, as it did in both the Wang, Liu, Zhan, and Shi (2010) study of work-family conflict and drinking and the Allen and Armstrong (2006) study of workfamily conflict, diet, and physical activity. It is also possible that the weekly time frame was too broad to precisely capture patterns of reactivity, a possibility that will be discussed further in the context of the study limitations. Having demonstrated that some variability existed in nurses' individual-level relationships between their weekly stressors and health behaviors in the five cases discussed here, I then turned to the question of what that reactivity means for health and well-being.

\section{Reactivity as a Predictor}

My second hypothesis was designed to test the proposition that variable patterns of reactivity might be associated with health and well-being outcomes. Logically it seems that this might be particularly likely to be the case if that reactivity is itself a mechanism through which those outcomes can come about. For instance, because exercise itself has health benefits, nurses who exercise more when they encounter more stressors might be expected to have better health and well-being later than those who exercise less when they encounter more stressors. I thus approached my investigation of Hypothesis 2 with the expectation that nurses whose health behaviors tended to improve in times when they encountered more stressors would have better outcomes than those whose health behaviors worsened in those circumstances. Where significant relationships emerged between reactivity and outcomes, that was generally true, however significant relationships were not found for all types of reactivity, and in one case a significant relationship directly contradicted my expectations. It is worth keeping in mind during the following discussion of results that, in the absence of information about 
the temporal ordering within week of stressors and behaviors, it is possible for each of the associations described below that the behavior changes preceded the stressors rather than the reverse. I will address each outcome examined under Hypothesis 2 in turn below.

Depression scores. Nurses' reactivity slopes for Demands-Exercise Days and Negative Events-Exercise Days were both significant predictors of depression scores at Wave 2. Additionally, the reactivity slope for Negative Events-Exercise Minutes approached significance as a predictor. In all three cases, nurses who exercised more than usual in weeks in which they encountered more stressors than usual overall had lower depression scores at Wave 2, and those who exercised less than usual in such weeks had higher depression scores. This pattern is consistent with existing literature on the mental health benefits of exercise (Centers for Disease Control and Prevention, 2011; Thayer et al., 1994).

The majority of nurses had negative stressor-exercise slopes $(80.3 \%$ for Demands-Exercise Days, 77.4\% for Negative Events-Exercise Days, and 63.5\% for Negative Events-Exercise Minutes), which is consistent with previous findings that greater job demands are associated with a decreased likelihood of following through on intentions to exercise (Payne et al., 2010). For the minority who increased their exercise during stressful weeks, the accompanying reduction in depression scores might represent successful use of exercise as a coping mechanism. Weeks in which nurses encountered more stressors than usual likely presented a variety of potential obstacles to getting regular exercise, such as time constraints, lack of energy, and stressor-related self-control failure. However, the higher depression scores associated with reduced exercise 
underscore the importance of, at minimum, maintaining one's normal exercise habits in the face of increased stressors, or, ideally, exercising more than usual during such times.

Satisfaction with life. I found additional evidence for the benefits of exercise in my examination of satisfaction with life. Specifically, the reactivity slope for ConflictsExercise Days was significant and its relationship to satisfaction with life was consistent with my proposition that those whose health behaviors improved during times of greater stressors would have better outcomes. Nurses who exercised more than usual in weeks of greater conflicts than usual had greater satisfaction with life; those who exercised less than usual in such weeks had lower satisfaction with life. Similar to the pattern for depression described above, this suggests that exercising to cope may be a successful strategy to adopt during times of more conflicts at work. Also similar to the findings for depression, the majority of nurses $(65.0 \%)$ tended to decrease their exercise during weeks with more conflicts rather than increase it.

Existing research on the effects of exercise has focused primarily on either the immediate benefits of exercise on mood (e.g., Petruzzello et al., 1991; Thayer et al., 1994) or its longer-term benefits for health (e.g., Colditz et al., 1997; Lee et al., 2012), and diary studies have focused primarily on changes in exercise behavior without examining the relationship between those changes and later outcomes (e.g., Newman et al., 2007; Steptoe et al., 1998). This dissertation builds on those approaches by presenting a unique examination of both dynamic patterns of stressors and exercise and how those patterns relate to later outcomes. Although research on the mental health and well-being benefits of exercise typically focuses on mood, anxiety, or depression rather than satisfaction with life, this finding could be contextualized within the positive 
psychology process known as "broaden-and-build" (Fredrickson, 1998, 2001; Tugade et al., 2004), in which positive moods can be used as a resource that both improves future coping and increases well-being. Exercise generates such positive moods (Thayer et al., 1994), and thus broaden-and-build is one way in which its benefits may extend beyond the immediately experienced improvement in mood to contribute to overall satisfaction with life.

Perceived health. The benefits of exercising to cope suggested by the findings for depression scores and satisfaction with life also held for perceived health; there were significant relationships between perceived health and nurses' reactivity slopes for both Conflicts-Exercise Days and Negative Events-Exercise Days. Specifically, nurses who increased their exercise days in weeks when they encountered more stressors tended to have better perceived health at Wave 2. However, when participants' Wave 1 level of perceived health was included as a predictor, those slopes no longer significantly predicted perceived health, suggesting that reactivity in the form of greater exercise does not in fact relate to increases in perceived health over the months of this study, it simply relates to higher levels of it at Wave 2, and conversely reactivity in the form of less exercise relates to lower levels of perceived health. Whether or not this kind of reactivity relates to changes in health over a longer period of time is a question for future research. As with the previously discussed findings, the majority of nurses actually exercised less in weeks of more conflicts $(65.0 \%)$ or negative events $(77.4 \%)$, therefore only a third or less of the sample received the potential physical health benefits of exercising to cope.

In contrast to the findings for exercise, the relationship between perceived health and the slope for Conflicts-Drinking Days was opposite to that predicted; increases in 
drinking days in weeks when conflicts were greater were associated with better perceived health at Wave 2, and decreases in drinking days conversely associated with poorer perceived health. In this case, the fact that the majority of nurses $(76.5 \%)$ had negative reactivity slope values indicates that most nurses drank less during higher conflict weeks and, as described above, the nurses whose slopes were positive had only very small increases. This may help to shed light on this finding.

Although drinking to cope has been found to be associated with the development of alcohol problems (Cooper et al., 1988), drinking at the relatively low levels engaged in by most participants who drank should be, in general, beneficial to longer-term health (Ashley et al., 1997; Colditz et al., 1997), and drinking to cope may in fact have some mental health benefits among moderate drinkers who are psychologically healthy (Mohr, Brannan, et al., 2010).

Given the low level of drinking in this sample in general, increases from participants' usual amount of drinking that are small in magnitude would still fall within the range of moderate drinking that has many health benefits. Decreases from those levels might be associated with lower perceived health for a variety of reasons. Anyone whose decrease took their drinking from a low level to none at all would not gain the health benefits of moderate drinking during the time when they did not drink. Moreover, if their normal pattern involved social drinking, the reduced number of drinking days could represent not only a reduction in consumption, but also a reduction in social time and thus social support that could have been beneficial during such a period of more stressors (S. Cohen \& Hoberman, 1983), although a similar pattern of poorer outcomes associated with decreased drinking might be expected to have emerged on the more 
mental health-oriented outcomes if that were the case. As described previously with regards to exercise, these findings are not representative of a change in health from Wave 1 to Wave 2, thus it may be that other factors account for both the lower level of perceived health among those who reduced their drinking and for that reactivity. Further research is necessary to determine whether or not reactivity in the form of decreased drinking is generally harmful to perceived health.

Summary. Some of the types of reactivity examined did significantly predict later outcomes, although one significant reactivity-outcome relationship was not in the direction expected. Each of the five reactivity slopes was a significant or approaching significant predictor in at least one model. Moreover, the slopes that were significant added explanatory power to their models above and beyond that of previously studied predictors of those outcomes such as age, sex, and neuroticism. In the cases where reactivity did not predict outcomes, it may be that those types of reactivity do not in fact impact those outcomes, or that repeated measures of those outcomes may be needed to fully understand whether they are improving or declining, and if so, whether or not reactivity relates to that change. Throughout, exercise patterns emerged as an important factor in well-being.

Reactivity to demands and general negative events in the form of increased exercise was associated with lower depression scores, reactivity to conflicts in the form of increased exercise was associated with greater satisfaction with life, and reactivity to conflicts and negative events in the form of increased exercise were both associated with better perceived health, suggesting together that increasing exercise when encountering 
increased stressors may be beneficial for mental and physical health and subjective wellbeing. Thus it seems reasonable to recommend exercise as a coping mechanism.

\section{Limitations and Suggestions for Future Studies}

Some limitations in measurement and methodology should be considered when interpreting the results of this dissertation and in the design of future studies. This dissertation measures events and behaviors on the weekly level, and thus the temporal ordering of them within weeks cannot be disentangled. It is possible that the health behavior being recorded actually occurred before the stressors, in which case the stressors could not have caused the health behavior change; in fact, the health behavior change could have led to the stressors, for example, if drinking more alcohol than usual led to impaired work performance the next day. Mood, one frequently used measure of reactivity that has been found to be linked to physical and mental health outcomes (Charles et al., 2013; Piazza et al., 2012), was not measured in this study and therefore is not available for analysis; however the attention paid to the development of the event measure lends support to the idea that the events are likely to be appraised negatively, making them likely to negatively impact mood. To test whether or not mood is being affected by stressors, future studies should consider including mood alongside the use of frequently collected data points, ideally multiple times per day.

More frequent measurements such as these would allow for more precise temporal examination of the sequencing of events and individuals' behavioral or emotional reactions to them, thereby ruling out the possibility that the behavior changes caused the stressors. Such measurements would allow future studies to address an additional possibility — that the quantity of events that occurred was also important, for 
instance, if one nurse's conflicts were greater than usual for a given week because of only one very bad day whereas another nurse had greater conflicts than usual for the week because of several consecutive bad days, it seems possible that they would be differentially affected. Although the weekly timeframe of measurement in this study both accommodated nurses' busy schedules, thus encouraging full weekly participation, and allowed for a better snapshot of patterns of stressors and behavior than a longer period of retrospection would have, that measurement frame of both health behaviors and work stressors does not allow for a daily level of analysis. Because of these limitations, this dissertation's findings should therefore be viewed as suggestive of relationships between events and behavior on a weekly level rather than a more granular level.

Those events and behaviors were both self-reported, and are therefore subject to the self-presentation and social desirability biases present in such data (Crowne \& Marlowe, 1960). However I believe the relatively anonymous, low-involvement nature of the data collection minimized that potential. Most participants never spoke to an ONRP staff member, and were simply responding to emails and survey links. This might have increased their feeling that the data was anonymous and thus their willingness to reveal potentially sensitive information such as alcohol consumption (Kreuter, Presser, \& Tourangeau, 2009). On the other hand, nurses were recruited primarily through contact from their union, an organization that actively advocates for changes in policy to improve nurses' working lives. Thus, despite instruction from the researchers that we were looking for honest opinions and information, they may have felt that this study, done in cooperation with their union, was an opportunity to air grievances about their work environment and thus might have over-reported negative work events. 
Some studies of negative events include positive events to test the direct physical or mental health benefits of positive events (S. Cohen \& Hoberman, 1983; Salovey, Rothman, Detweiler, \& Steward, 2000). Others consider the notion that positive events might reduce or prevent negative consequences of negative events (Fredrickson, Mancuso, Branigan, \& Tugade, 2000; Tugade et al., 2004), for instance feeling overloaded by a busier than usual shift at work might be less distressing to a nurse who also felt happy about having helped to save the life of a patient earlier that day. Disentangling the effects of negative and positive events adds another layer of complexity to analyses such as those conducted here. For instance, in the Mohr et al. (2013) study of mood-drinking slopes as predictors, positive mood-drinking alone slopes were actually associated with some poorer outcomes later on. Reactivity to positive events might also be taking place in this sample, and its effects on outcomes remain unknown for now. Given that this dissertation is an initial exploration of the relationship between reactivity and outcomes, I opted not to include analysis of positive events, but they could be considered for inclusion in future study. Other protective factors such as social support or other resources an individual may have to draw on when stressors occur could also be impacting both reactivity and outcomes, and could also be included for consideration in future research.

Future studies should also include measurement of outcome variables at more than one timepoint. In this study, depression and life satisfaction were not tested at Wave 1, and therefore it cannot be determined whether individuals' scores at follow-up differ from their scores four months prior - in other words, whether or not change has occurred. Therefore the weekly reactivity seen here cannot be assumed to be associated with either 
increases or decreases in those scores. However, the study start date was not linked to a particular time or event such as the start of a treatment program, and no formal intervention was carried out; thus whatever individual patterns of reactivity and wellbeing exist in this sample at the Wave 2 survey likely began well before the date of the initial interview. Satisfaction with life has been shown to be relatively temporally stable (Lucas, 2008; Suh et al., 1996), particularly if no intervention occurs between measurements, so a four-month window would likely have been insufficient to see reactivity-related change within the timeframe of the study. Instead, it is possible that the relationships between reactivity and satisfaction with life described here relate to an ongoing pattern for individuals that began long before this study took place. Test-retest CES-D scores administered with a similar gap in time have been found to be moderately correlated (approximately .5), and thus changes that could have been found over this time frame would likely have been relatively minor (Radloff, 1977). It seems likely that, as with satisfaction with life, the relationships described here between reactivity and depression scores began before this study took place. In fact, depression itself may impact reactivity, although the direction of the depression-reactivity relationship has been inconsistent in previous studies (Peeters et al., 2003; Steger \& Kashdan, 2009). Overall, the relatively low likelihood that reactivity led to change in these outcomes over the course of this study does not mean that reactivity does not play a role in the levels of those outcomes, simply that a causal relationship between reactivity and change in those outcomes cannot be demonstrated with this data.

Nurses were limited to those working in hospitals within the state of Oregon. Any results of this dissertation should therefore be replicated with other types of workers 
in other types of work environments in other regions. Moreover, although the restriction of the data collection to nursing events allows for the examination of similar stressors across individuals, it also excludes other potential sources of variation, such as family stressors or work-family conflict, that may affect an individual's working life and health behaviors. Finally, this sample is predominantly female, as is the nursing profession. Although no sex differences were found in the regression models tested for Hypothesis 2, it cannot be assumed that reactivity would operate the same way in a male or genderbalanced sample as it does here. However the results of this dissertation may be viewed as potentially representative of the patterns that exist among female nurses.

\section{Contributions, Implications, and Conclusion}

Strengths of this dissertation include elements of the sample, measures, and analyses. The geographic diversity of the sample, which was drawn statewide rather than from one particular hospital or city, should offer greater generalizability than a more geographically narrow sample might. Nurses as a population of interest are also likely to have experienced a number of negative work events, thereby providing a sample size and event frequency adequate for these analyses. This study's focus on nursing work and the ONRP staff's intensive development process for the negative events examined here should have combined to generate a relatively comprehensive list of the important negative events nurses are likely to encounter in their work. Moreover, longitudinal studies of such events, reactivity, and outcomes are still relatively rare, and thus the longitudinal nature of this data is another strength of this dissertation. For instance, in the case of exercise, research on patterns has focused primarily on whether or not patterns change in response to stressors (e.g., Newman et al., 2007), and research on outcomes has 
tended to focus on the short or long-term effects of exercise itself such as improvements in mood or reduced risk of disease (e.g., Thayer et al., 1994). I am unaware of any previous studies attempting to draw a link between changing patterns of exercise (reactivity) and outcomes, a link which cannot be investigated without longitudinal data collection. Finally, the relatively new slopes-as-predictors analysis technique, which offers the potential to open new research questions in many fields, has not been applied to studies of reactivity and health behavior, with the exception of drinking in the Mohr et al. (2013) paper, and thus this dissertation represents an early endeavor in that area.

With this dissertation I have attempted to contribute to the body of scientific knowledge in three major ways: by exploring linkages between immediate and longerterm effects of stressors, by illuminating one potential mechanism through which those longer-term effects might happen, and by adding to the understanding of nurses' wellbeing. Behavioral reactivity was indeed present in this sample in multiple domains, and although I found fewer relationships between that reactivity and outcomes than hypothesized, some relationships did exist, thereby fulfilling my purpose of exploring linkages between immediate and longer-term effects of stressors. Moreover, reactivity did, in some cases, demonstrate the potential to be a mechanism through which stressors led to later outcomes, such as the case of Conflicts-Exercise Days and satisfaction with life. It is possible that the decrease in days of exercise some nurses demonstrated during higher-conflict weeks was in fact part of the reason why their later satisfaction with life was lower, as they obtained fewer of the mental health benefits of exercise over time than their peers who exercised to cope with conflicts. Further research on reactivity can 
continue to explore these and other potential ways in which reactivity might link stressors and outcomes.

This dissertation suggests that reactivity to stressors in the form of health behavior change may be an important mechanism through which negative events impact nurses' health and well-being. The consistent association between reactivity in the form of increased exercise and better mental health and satisfaction with life also suggests a pathway for intervention. Exercise is already an encouraged health behavior, a fact that nurses are no doubt aware of, but among the important determinants of exercise are overall cultural norms and social supports in the work environment (Bauman et al., 2012). Moreover, organizations are an important part of ecological models of health promotion, which state that changes in health behavior can be influenced and supported at both the individual and environmental level (McLeroy, Bibeau, Steckler, \& Glanz, 1988).

In a large meta-analysis of nearly 40 years' worth of research on workplace-based physical activity interventions, Conn, Hafdahl, Cooper, Brown, and Lusk (2009) found that such interventions could be helpful for employee fitness, health, and job stress, as well as for more specifically work-focused outcomes such as attendance. They found that interventions delivered during paid work time seemed to be more effective for fitness than those that were not, as were those that employees helped design and deliver. In addition to the usual information about the physical and mental health benefits of exercise that are commonly included in exercise promotion campaigns and programs, participants in such interventions could also be informed of the potential benefits of 
exercising to cope - that is, not just maintaining regular exercise habits, but engaging in exercise as an intentional way to assist them in dealing with the effects of stressors.

Taking into consideration the fact that the majority of nurses in this study tended to decrease their exercise days in weeks of greater stressors, adding exercise to cope into their schedule might be a more advanced goal for many, but given the associations found in this dissertation between decreased exercise and poorer outcomes, support in maintaining their existing exercise routines even during times when they encounter many work stressors would be an important first step for many nurses. Providing support within the work environment and encouraging an organizational culture in which the potential benefits of exercise for coping with stressors are emphasized might encourage more nurses to exercise to cope. If nurses could be assisted in this way in coping with shorter-term, more commonly encountered negative events, it might help to not only prevent negative health and well-being outcomes, but also to contribute to positive ones. These principles could be applied to non-nursing populations as well.

To date, most longitudinal studies of stressors and health outcomes have focused on the occurrence and characteristics of stressors as predictors. Studies of reactivity, on the other hand, have typically focused on its short-term effects rather than taking a longitudinal approach. This dissertation has built on those approaches by examining immediate reactions to naturalistically encountered stressors in the form of behavioral reactivity and taking advantage of novel statistical techniques to test the relationship between that reactivity and later outcomes. Findings suggest that reactivity in the form of exercising to cope has benefits for mental health and satisfaction with life. This dissertation has explored linkages between reactivity and health and contributed to the 
understanding of nurses' well-being. It is well-documented that stressors themselves can predict later health and well-being, and this dissertation has added to that some new evidence that reactivity to those stressors is also predictive of health and well-being, thereby suggesting one pathway for how stressors lead to those outcomes. Based on the findings presented here, hospitals might be encouraged to increase their efforts to support nurses' exercise habits and encourage the use of exercise as a coping technique; in general, the study of behavioral reactivity to stressors may lead to additional recommendations for intervention in the future. 
Table 1. Hypothesized Reactivity-Outcome Relationships

\begin{tabular}{cll}
\hline \multicolumn{1}{c}{ Hypothesis } & \multicolumn{1}{c}{ Form of Reactivity } & \multicolumn{1}{c}{ Expected Outcome } \\
\hline 2a: Depression Scores & Decreased exercise & Higher CES-D scores \\
& Increased exercise & Lower CES-D scores \\
& Poorer eating & Higher CES-D scores \\
& Better eating & Lower CES-D scores \\
& More drinking & Higher CES-D scores \\
& Less drinking & Lower CES-D scores \\
\hline 2b: Satisfaction with Life & Decreased exercise & Lower SWLS scores \\
& Increased exercise & Higher SWLS scores \\
& Poorer eating & Lower SWLS scores \\
& Better eating & Higher SWLS scores \\
& More drinking & Lower SWLS scores \\
& Less drinking & Higher SWLS scores \\
\hline 2c: Perceived Health & Decreased exercise & Poorer perceived health \\
& Increased exercise & Better perceived health \\
& Poorer eating & Poorer perceived health \\
& Better eating & Better perceived health \\
& More drinking & Poorer perceived health \\
& Less drinking & Better perceived health \\
\hline
\end{tabular}

Note: More/less drinking measured as days or drinks; more/less exercise measured as minutes or days. 
Table 2: Correlations of Slope Components

\begin{tabular}{|c|c|c|c|c|c|c|c|c|c|c|c|c|}
\hline & 2 & 3 & 4 & 5 & 6 & 7 & 8 & Age & Neur. & SWLS & $\begin{array}{l}\text { CES- } \\
\mathrm{D}\end{array}$ & $\begin{array}{l}\text { Perceived } \\
\text { Health }\end{array}$ \\
\hline $\begin{array}{l}\text { 1. Ate } \\
\text { Healthy }\end{array}$ & $.46 * * *$ & $.36 * * *$ & .11 & .05 & .09 & .07 & .12 & $.15^{\dagger}$ & -.14 & $.24 * *$ & $-.18 *$ & $.49 * * *$ \\
\hline $\begin{array}{l}\text { 2. Exercise } \\
\text { Days }\end{array}$ & & $.67 * * *$ & .06 & .10 & $.15^{\dagger}$ & .12 & $.23 * *$ & .11 & $-.14^{\dagger}$ & $.23 * *$ & -.08 & $.45 * * *$ \\
\hline $\begin{array}{l}\text { 3. Exercise } \\
\text { Mins }\end{array}$ & & & .10 & $.23 *$ & $.23 * *$ & $.20 *$ & $.28 * *$ & -.04 & $-.15^{\dagger}$ & .10 & .02 & $.31 * * *$ \\
\hline $\begin{array}{l}\text { 4. Drinking } \\
\text { Days }\end{array}$ & & & & $.68 * * *$ & .04 & .04 & .05 & $.22 *$ & -.03 & .14 & -.08 & .18 \\
\hline $\begin{array}{l}\text { 5. Number of } \\
\text { Drinks }\end{array}$ & & & & & .00 & .01 & -.03 & .07 & .00 & .03 & .03 & .08 \\
\hline 6. All NE & & & & & & $\mathrm{X}$ & $\mathrm{X}$ & -.02 & .09 & -.14 & $.24 * *$ & -.09 \\
\hline 7. Demands & & & & & & & $.68 * * *$ & .02 & .11 & $-.18 *$ & $.24 * *$ & -.12 \\
\hline 8. Conflicts & & & & & & & & -.14 & -.01 & .04 & $.16^{\dagger}$ & .04 \\
\hline
\end{tabular}


Table 3: Slopes and Fixed Effects (H1)

\begin{tabular}{lcc}
\hline Parameter/Slope & Fixed Effect (SD) & Variance Component (SE) \\
\hline Negative Events and: & $.07(.07)$ & $.05(.22)$ \\
Ate Healthy & $-.29(.19)$ & $\mathbf{1 . 1 2}(\mathbf{1 . 0 6}) * *$ \\
Exercise Days & $-.11(.17)$ & $\mathbf{. 5 0 ( . 7 0 ) * *}$ \\
Exercise Minutes & $-.06(.09)$ & $.00(.04)$ \\
Drinking Days & $-.18(.08)^{*}$ & $.01(.11)$ \\
Number of Drinks & & \\
Conflicts and: & $.05(.10)$ & $.22(.46)$ \\
Ate Healthy & $-.23(.23)$ & $\mathbf{1 . 5 8}(\mathbf{1 . 2 6})^{*}$ \\
Exercise Days & $.17(.22)$ & $1.17(1.08)^{\dagger}$ \\
Exercise Minutes & $-.19(.08)^{*}$ &. $\mathbf{0 5}(. \mathbf{2 2})^{* *}$ \\
Drinking Days & $-.32(.14)^{*}$ & $.17(.41)$ \\
Number of Drinks & & \\
Demands and: & $.04(.05)$ & $.01(.11)^{\dagger}$ \\
Ate Healthy & $-.21(.14)$ & $.55(.74)^{*}$ \\
Exercise Days & $-.12(.12)$ & $.15(.39)$ \\
Exercise Minutes & $-.02(.06)$ & $.00(.05)$ \\
Drinking Days & $-.10(.05) \dagger$ & $.00(.03)$ \\
Number of Drinks & &
\end{tabular}

Note: ${ }^{\dagger} p<.10,{ }^{*} p<.05,{ }^{* *} p<.01$. 
Table 4: Slope, Outcome, and Covariate Descriptive Statistics

\begin{tabular}{lccc}
\hline Slope & M (SD) & Minimum & Maximum \\
\hline Negative Events-Exercise Minutes & $-.11(.27)$ & -1.66 & .66 \\
Negative Events-Exercise Days & $-.29(.50)$ & -1.90 & 1.87 \\
Conflicts-Exercise Days & $-.23(.73)$ & -1.45 & 2.26 \\
Conflicts-Drinking Days & $-.19(.21)$ & -.65 & .15 \\
Demands-Exercise Days & $-.21(.33)$ & -1.33 & 1.12 \\
\hline Neuroticism & $12.78(3.20)$ & 5.00 & 21.00 \\
Age & $44.22(10.85)$ & 22.00 & 66.00 \\
\hline CES-D Scores & $6.53(4.25)$ & 0.00 & 21.00 \\
SWLS Scores & $25.24(6.24)$ & 6.00 & 35.00 \\
Perceived Health & $3.75(.89)$ & 2.00 & 5.00
\end{tabular}

Note: slopes $\mathrm{N}=137$; except Conflicts-Drinking Days, where $\mathrm{N}=98$. Demographics $\mathrm{N}=135$. Outcome variables $\mathrm{N}=128$. 
Table 5: Correlations of Slopes and Outcomes

\begin{tabular}{|c|c|c|c|c|c|c|c|c|c|}
\hline & 2 & 3 & 4 & 5 & 6 & 7 & 8 & 9 & 10 \\
\hline $\begin{array}{l}\text { 1. Negative } \\
\text { Events-Exercise } \\
\text { Minutes }\end{array}$ & $.31 * * *$ & .00 & -.04 & $.37 * * *$ & .01 & .11 & -.01 & -.03 & -.05 \\
\hline $\begin{array}{l}\text { 2. Negative } \\
\text { Events-Exercise } \\
\text { Days }\end{array}$ & & $.61 * * *$ & -.04 & $.95 * * *$ & .06 & .09 & .07 & -.13 & $.19 *$ \\
\hline $\begin{array}{l}\text { 3. Conflicts- } \\
\text { Exercise Days }\end{array}$ & & & -.02 & $.35 * * *$ & .03 & -.08 & $.20 *$ & $-.17^{\dagger}$ & $.32 * * *$ \\
\hline $\begin{array}{l}\text { 4. Conflicts- } \\
\text { Drinking Days }\end{array}$ & & & & -.02 & .11 & -.06 & .13 & -.08 & $.20^{\dagger}$ \\
\hline $\begin{array}{l}\text { 5. Demands- } \\
\text { Exercise Days }\end{array}$ & & & & & .08 & .13 & .01 & -.10 & .14 \\
\hline $\begin{array}{l}\text { 6. Age } \\
\text { 7. Neuroticism }\end{array}$ & & & & & & -.10 & $\begin{array}{l}-.11 \\
-.37 * *\end{array}$ & $\begin{array}{l}.04 \\
.47 * *\end{array}$ & $\begin{array}{c}.21^{*} \\
-.26^{* *}\end{array}$ \\
\hline $\begin{array}{l}\text { 8. SWLS } \\
\text { 9. CES-D } \\
\text { 10. Perceived } \\
\text { Health }\end{array}$ & & & & & & & & $-.51 * * *$ & $\begin{array}{l}.51 * * * \\
-.34 * *\end{array}$ \\
\hline
\end{tabular}


Table 6: Regressions of CES-D Scores on Slopes (H2a)

\begin{tabular}{|c|c|c|c|}
\hline Predictors & $\mathrm{b}$ & $\mathrm{t}$ & $\mathrm{SPR}^{2}$ \\
\hline \multicolumn{4}{|l|}{ Model $1 * * *$} \\
\hline Sex & -1.46 & -.90 & .00 \\
\hline Age & .01 & .38 & .00 \\
\hline Neuroticism & .66 & $6.07 * * *$ & .24 \\
\hline Demands-Exercise Days & -2.15 & $-2.09 *$ & .03 \\
\hline \multicolumn{4}{|l|}{ Model 2*** } \\
\hline Sex & -1.62 & -1.00 & .01 \\
\hline Age & .01 & .34 & .00 \\
\hline Neuroticism & .66 & $6.06^{* * *}$ & .24 \\
\hline Negative Events-Exercise Days & -1.54 & $-2.28 *$ & .03 \\
\hline \multicolumn{4}{|l|}{ Model 3*** } \\
\hline Sex & -1.17 & -.73 & .00 \\
\hline Age & .01 & .34 & .00 \\
\hline Neuroticism & .66 & $6.02 * * *$ & .24 \\
\hline Negative Events-Exercise Minutes & -2.50 & $-1.84^{\dagger}$ & .02 \\
\hline \multicolumn{4}{|l|}{ Model 4*** } \\
\hline Sex & -.71 & -.35 & .00 \\
\hline Age & .02 & .55 & .00 \\
\hline Neuroticism & .69 & $4.67 * * *$ & .21 \\
\hline Conflicts-Drinking Days & -1.43 & -.71 & .00 \\
\hline \multicolumn{4}{|l|}{ Model $5 * * *$} \\
\hline Sex & -1.15 & -.71 & .00 \\
\hline Age & .01 & .24 & .00 \\
\hline Neuroticism & .62 & $5.64 * * *$ & .21 \\
\hline Conflicts-Exercise Days & -.71 & -1.54 & .02 \\
\hline
\end{tabular}

Note: ${ }^{\dagger} p<.10, * p<.05, * * p<.01 * * * p<.001 . \mathrm{SPR}^{2}=$ squared semi-partial correlation. 
Table 7: Regressions of SWLS Scores on Slopes (H2b)

\begin{tabular}{|c|c|c|c|}
\hline Predictors & $\mathrm{b}$ & $\mathrm{t}$ & $\mathrm{SPR}^{2}$ \\
\hline \multicolumn{4}{|l|}{ Model $1 * * *$} \\
\hline Sex & -.07 & -.03 & .00 \\
\hline Age & -.07 & -1.49 & .02 \\
\hline Neuroticism & -.69 & $-4.20^{* * *}$ & .12 \\
\hline Conflicts-Exercise Days & 1.50 & 2.16* & .03 \\
\hline \multicolumn{4}{|l|}{ Model 2** } \\
\hline Sex & -1.52 & -.56 & .00 \\
\hline Age & -.11 & $-1.90^{\dagger}$ & 03 \\
\hline Neuroticism & -.67 & $-3.41 * *$ & .11 \\
\hline Conflicts-Drinking Days & 4.00 & 1.49 & .02 \\
\hline \multicolumn{4}{|l|}{ Model 3** } \\
\hline Sex & -.43 & -.17 & .00 \\
\hline Age & -.08 & -1.50 & .02 \\
\hline Neuroticism & -.74 & $-4.39 * * *$ & .14 \\
\hline Demands-Exercise Days & 1.48 & .93 & .01 \\
\hline \multicolumn{4}{|l|}{ Model 4*** } \\
\hline Sex & -.14 & -.06 & .00 \\
\hline Age & -.08 & -1.51 & .02 \\
\hline Neuroticism & -.75 & $-4.44 * * *$ & .14 \\
\hline Negative Events-Exercise Days & 1.38 & 1.31 & .01 \\
\hline \multicolumn{4}{|l|}{ Model 5** } \\
\hline Sex & -.58 & -.24 & .00 \\
\hline Age & -.08 & -1.50 & .02 \\
\hline Neuroticism & -.75 & $-4.40 * * *$ & .14 \\
\hline Negative Events-Exercise Minutes & 2.00 & .95 & .01 \\
\hline
\end{tabular}

Note: ${ }^{\dagger} p<.10,{ }^{*} p<.05 * * p<.01 * * * p<.001 . \mathrm{SPR}^{2}=$ squared semi-partial correlation. 
Table 8: Regressions of Perceived Health on Slopes $(\mathrm{H} 2 \mathrm{c})$

\begin{tabular}{|c|c|c|c|}
\hline Predictors & $\mathrm{b}$ & $\mathrm{t}$ & $\mathrm{SPR}^{2}$ \\
\hline \multicolumn{4}{|l|}{ Model 1* } \\
\hline Sex & -.29 & -.68 & .00 \\
\hline Age & .02 & $1.94^{\dagger}$ & .04 \\
\hline Neuroticism & -.05 & $-1.68^{\dagger}$ & .03 \\
\hline Conflicts-Drinking Days & .85 & $2.03 *$ & .04 \\
\hline \multicolumn{4}{|l|}{ Model $2 * * *$} \\
\hline Sex & -.29 & -.85 & .00 \\
\hline Age & .02 & $2.21 *$ & .03 \\
\hline Neuroticism & -.06 & $-2.71 * *$ & .05 \\
\hline Conflicts-Exercise Days & .33 & $3.36 * *$ & .08 \\
\hline \multicolumn{4}{|l|}{ Model $3 * *$} \\
\hline Sex & -.29 & -.82 & .00 \\
\hline Age & .02 & $2.09 *$ & .03 \\
\hline Neuroticism & -.08 & $-3.12 * *$ & .07 \\
\hline Negative Events-Exercise Days & .33 & $2.17 *$ & .03 \\
\hline \multicolumn{4}{|l|}{ Model 4** } \\
\hline Sex & -.35 & -.97 & .01 \\
\hline Age & .02 & $2.05^{*}$ & .03 \\
\hline Neuroticism & -.08 & $-3.10 * *$ & .07 \\
\hline Demands-Exercise Days & .39 & $1.71^{\dagger}$ & .02 \\
\hline \multicolumn{4}{|l|}{ Model 5** } \\
\hline Sex & -.46 & -1.28 & .01 \\
\hline Age & .02 & $2.14 *$ & .03 \\
\hline Neuroticism & -.07 & $-2.84 * *$ & .06 \\
\hline Negative Events-Exercise Minutes & .10 & .34 & .00 \\
\hline
\end{tabular}

Note: ${ }^{\dagger} p<.10, * p<.05, * * p<.01 * * * p<.001 . \mathrm{SPR}^{2}=$ squared semi-partial correlation. 
Figure 1.

The transactional model of stress and coping

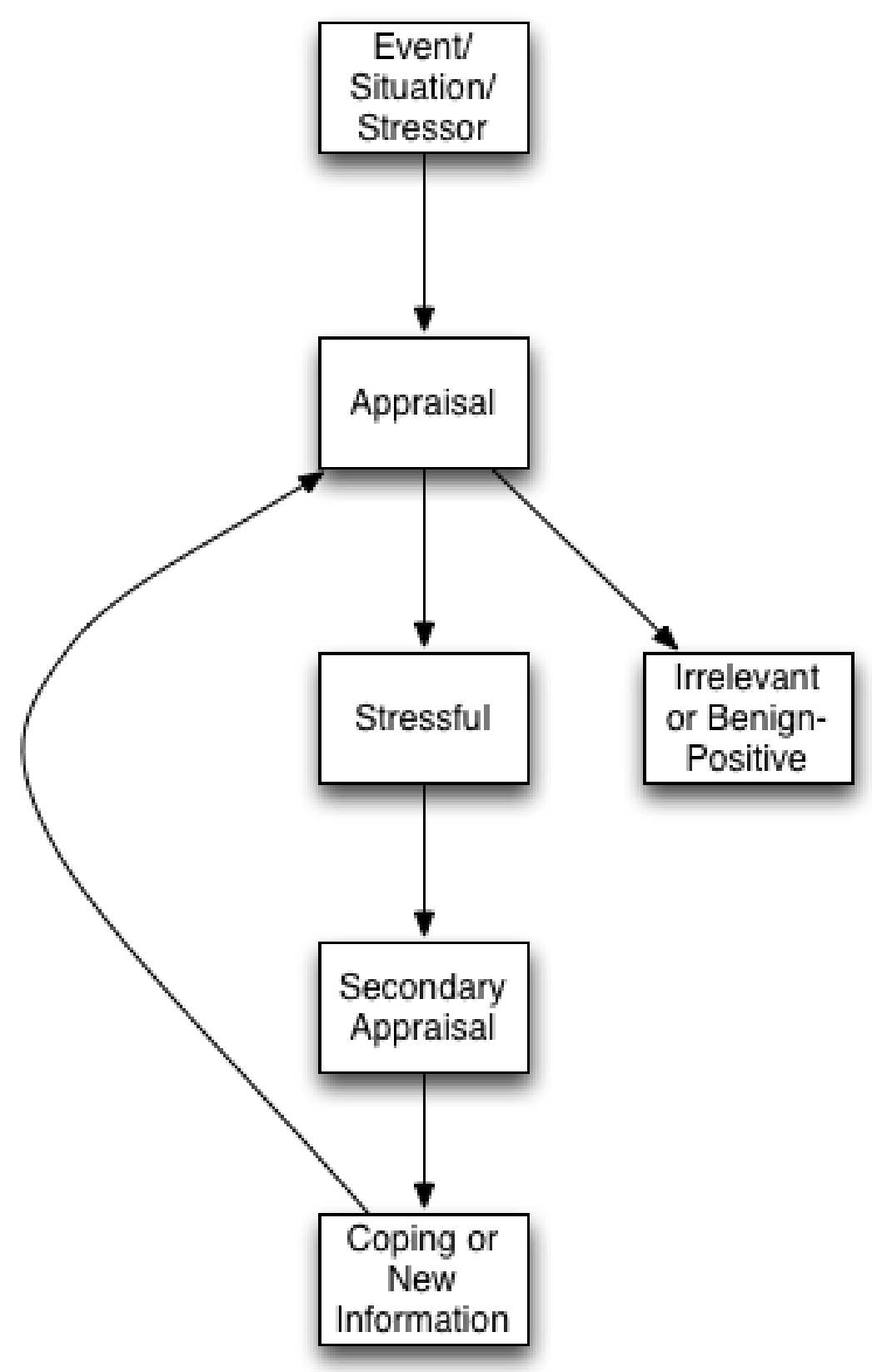


Figure 2.

Immediate and longer-term reactions to stressors

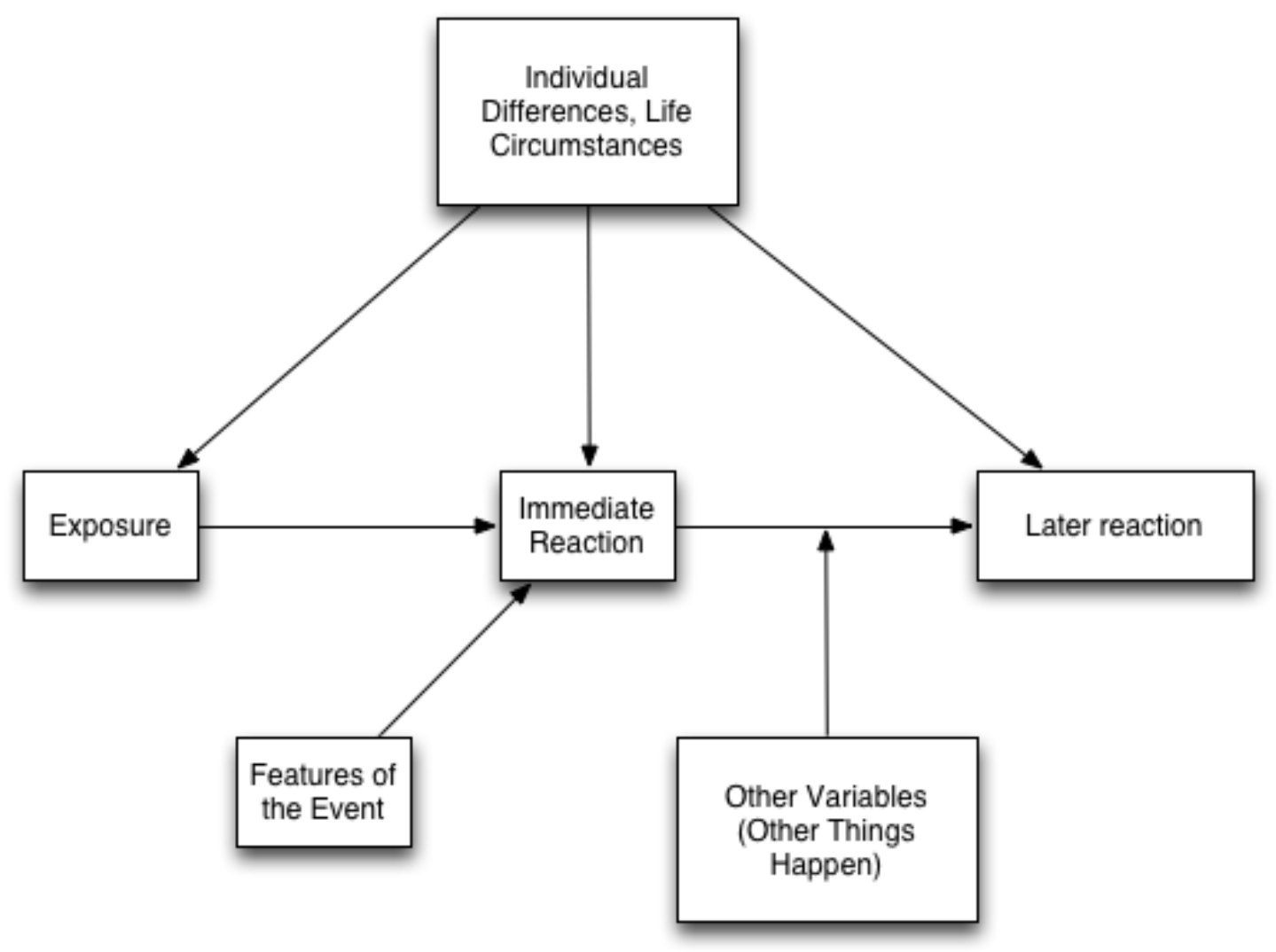


Figure 3.

Stressors and health behavior

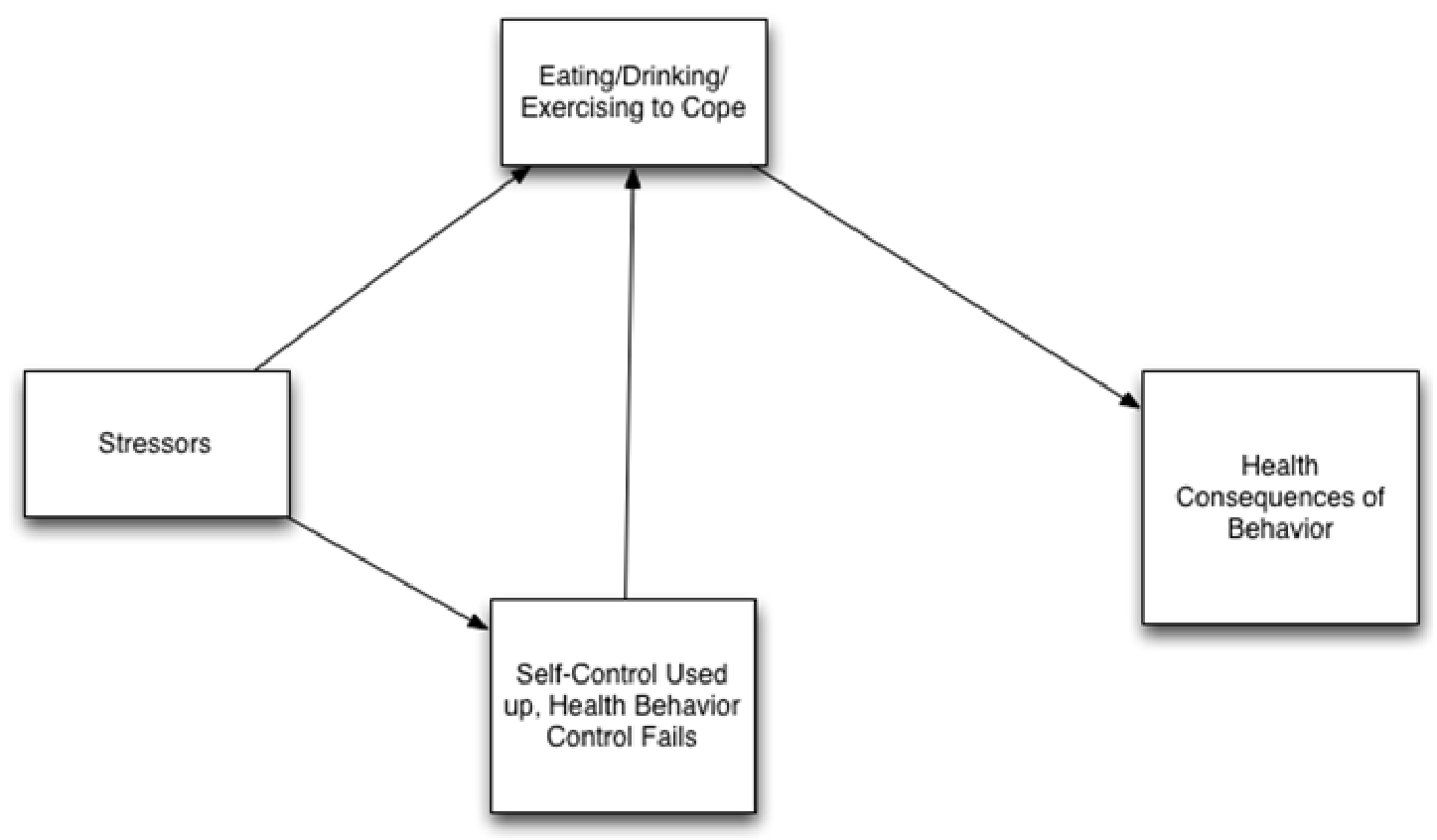


Figure 4.

The Oregon Nurse Retention Model

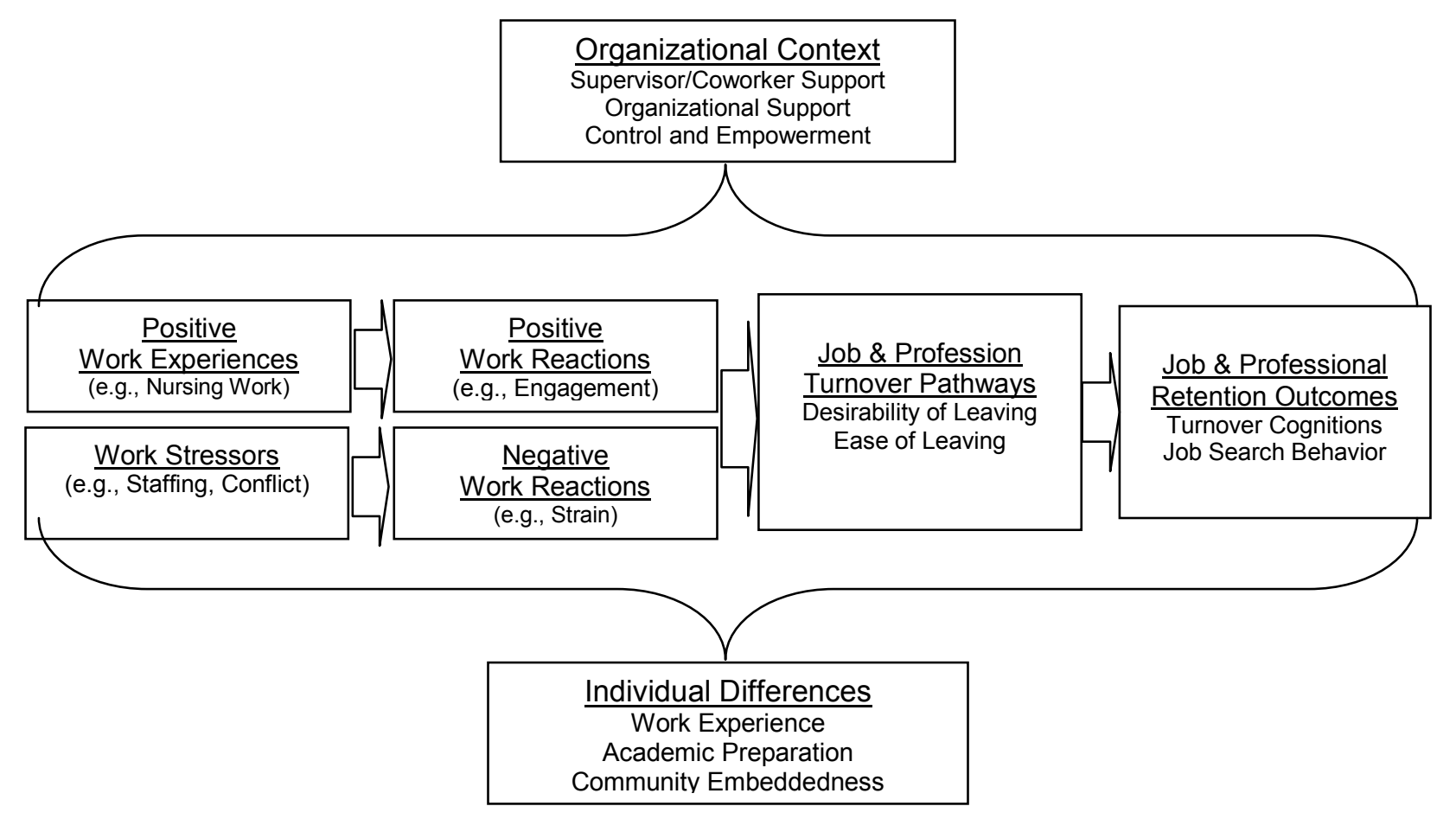

From Sinclair, et al. (2009). 
Figure 5a.

Mean Weekly Conflicts-Exercise Days Slopes

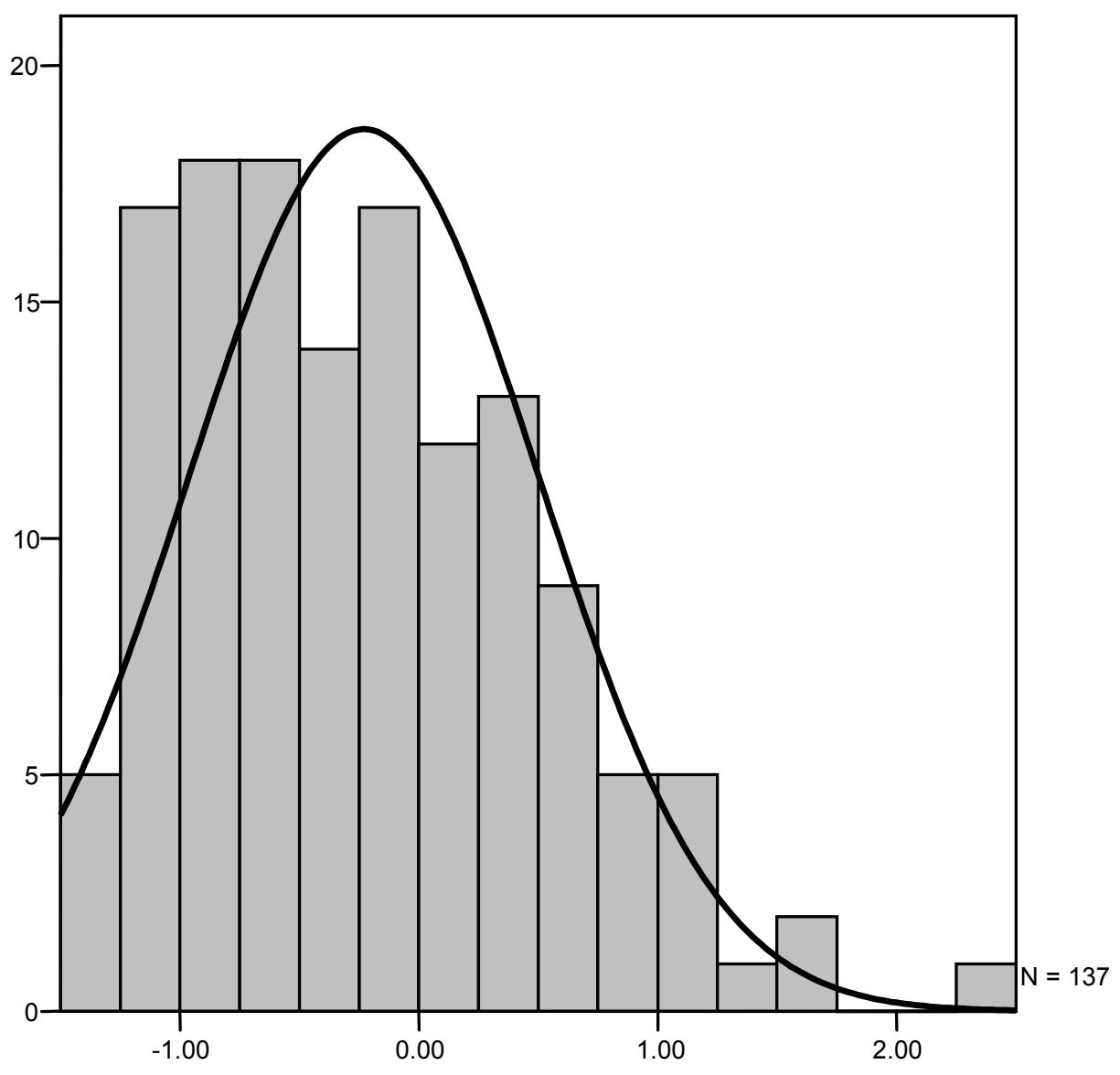


Figure $5 b$.

Mean Weekly Demands-Exercise Days Slopes

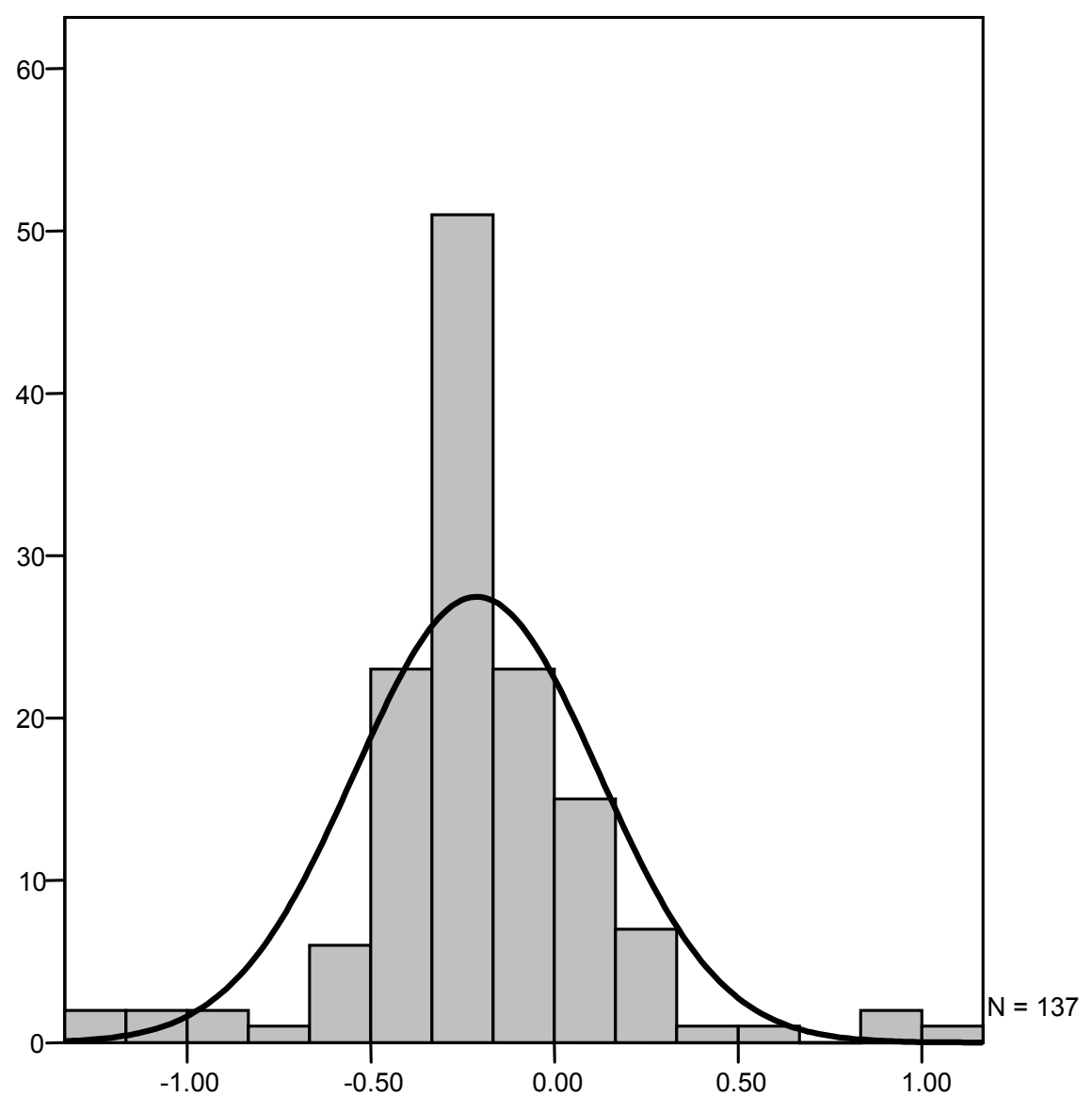


Figure 5c.

Mean Weekly Negative Events-Exercise Days Slopes

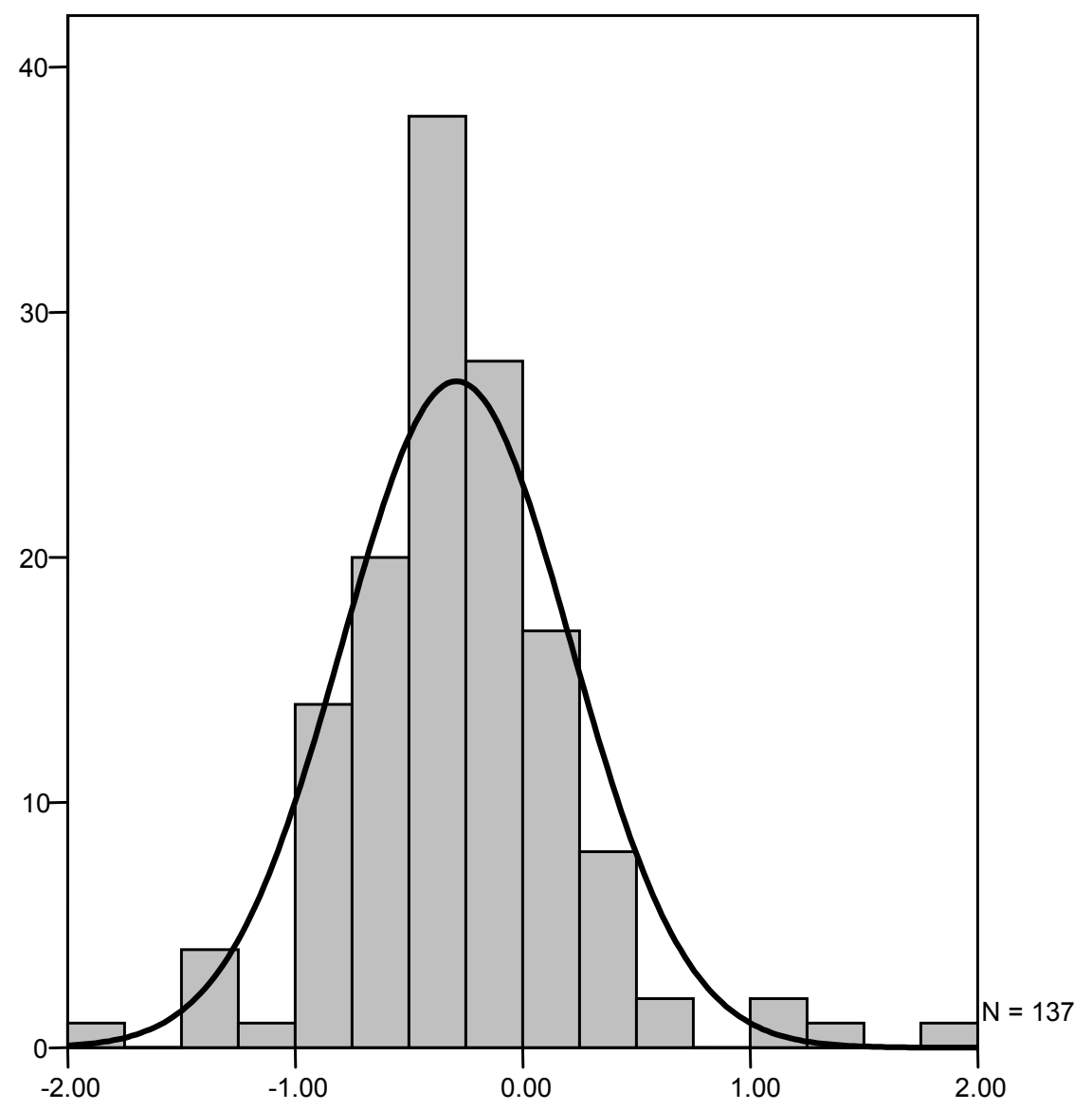


Figure 5d.

Mean Weekly Negative Events-Exercise Minutes Slopes

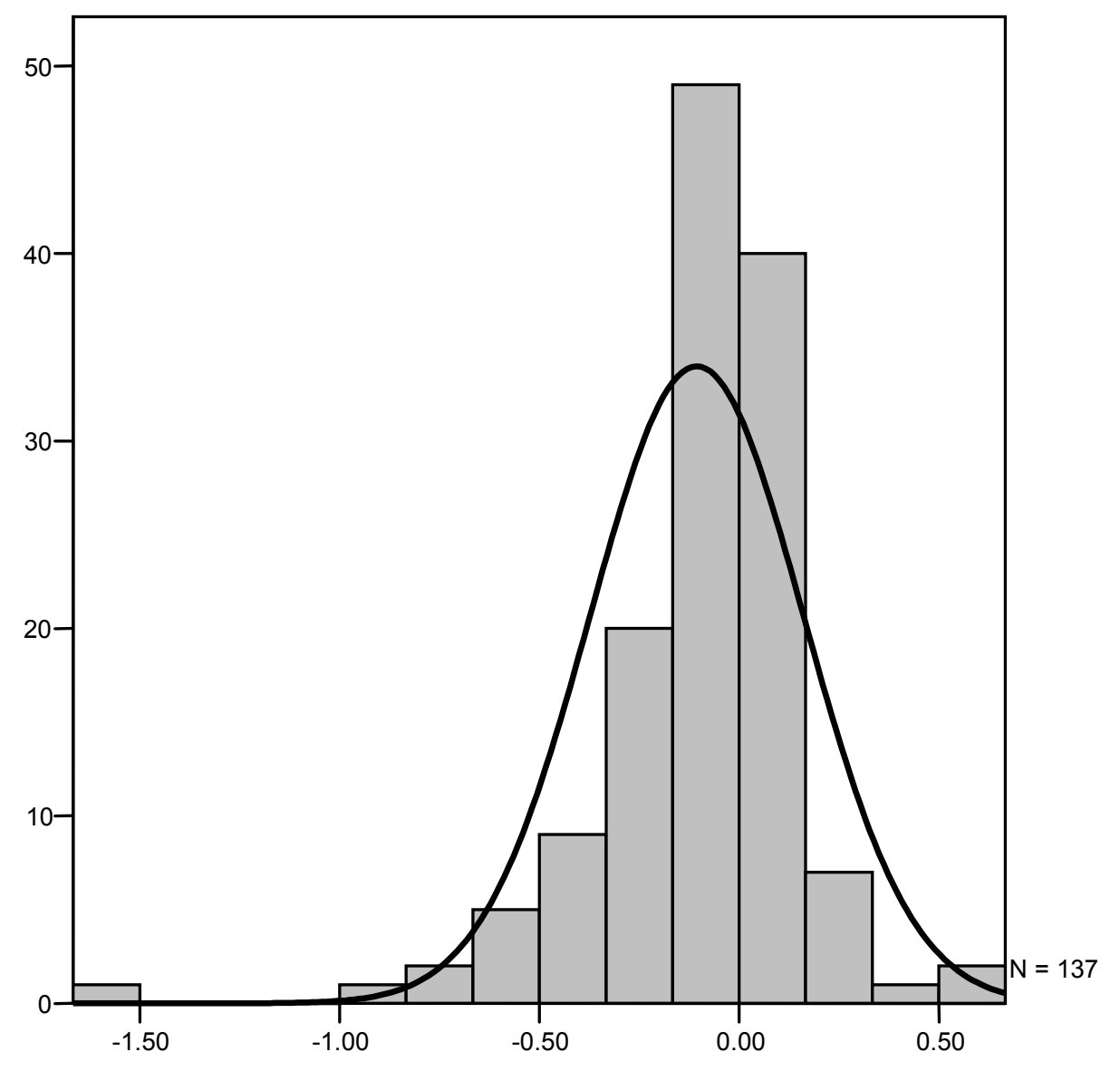


Figure 5e.

Mean Weekly Conflicts-Drinking Days Slopes

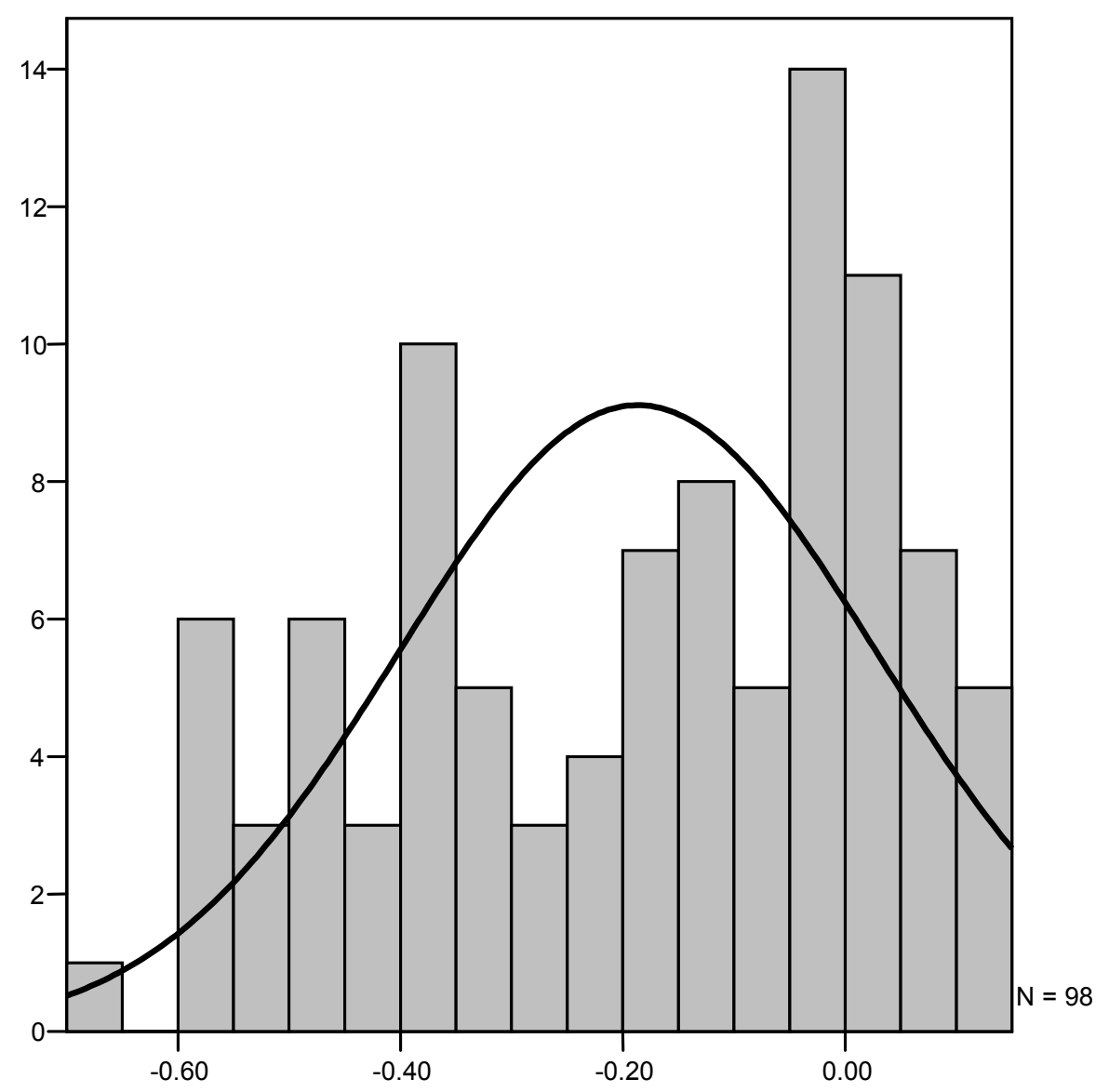




\section{References}

Affleck, G. G., Tennen, H., Urrows, S., \& Higgins, P. (1994). Person and contextual features of daily stress reactivity: Individual differences in relations of undesirable daily events with mood disturbance and chronic pain intensity. Journal of Personality and Social Psychology, 66, 329-340.

Allen, T. D., \& Armstrong, J. (2006). Further examination of the link between workfamily conflict and physical health: The role of health-related behaviors. American Behavioral Scientist, 49(9), 1204-1221. doi: $10.1177 / 0002764206286386$

Almeida, D. M. (2005). Resilience and vulnerability to daily stressors assessed via diary methods. Current Directions in Psychological Science, 14, 64-68. doi:10.1111/j.0963-7214.2005.00336.x

Almeida, D. M., \& Horn, M. C. (2004). Is daily life more stressful during middle adulthood? In How Healthy Are We? A National Study of Well-Being at Midlife. University of Chicago Press.

Almeida, D. M., Wethington, E., \& Kessler, R. C. (2002). The daily inventory of stressful events: An interview-based approach for measuring daily stressors. Assessment, 9, 41-55. doi:10.1177/1073191102091006

Armeli, S., Todd, M., \& Mohr, C. D. (2005). A daily process approach to individual differences in stress-related alcohol use. Journal of Personality, 73, 1657-1686. doi:10.1111/j.0022-3506.2005.00362.x

Armor, D. J., Polich, J. M., \& Stambul, H. B. (1978). Alcoholism and treatment. Retrieved from https://www.ncjrs.gov/App/Publications/abstract.aspx?ID=52064 
Ashley, M. J., Ferrence, R., Room, R., Bondy, S., Rehm, J., \& Single, E. (1997).

Moderate drinking and health: Implications of recent evidence. Canadian Family Physician, 43, 687-694.

Bauman, A. E., Reis, R. S., Sallis, J. F., Wells, J. C., Loos, R. J., \& Martin, B. W. (2012). Correlates of physical activity: Why are some people physically active and others not? The Lancet, 380, 258-271. doi:10.1016/S0140-6736(12)60735-1

Belsky, J., \& Pluess, M. (2009). Beyond diathesis stress: Differential susceptibility to environmental influences. Psychological Bulletin, 135, 885-908. doi:10.1037/a0017376

Billings, A. G., \& Moos, R. H. (1982). Stressful life events and symptoms: A longitudinal model. Health Psychology, 1, 99-117. doi:10.1037/0278-6133.1.2.99

Boehm, J. K., Peterson, C., Kivimaki, M., \& Kubzansky, L. (2011). A prospective study of positive psychological well-being and coronary heart disease. Health Psychology, 30, 259-267. doi:10.1037/a0023124

Bolger, N., DeLongis, A., Kessler, R. C., \& Schilling, E. A. (1989). Effects of daily stress on negative mood. Journal of Personality and Social Psychology, 57, 808-818. doi: $10.1037 / 0022-3514.57 .5 .808$

Bolger, N., \& Schilling, E. A. (1991). Personality and the problems of everyday life: The role of neuroticism in exposure and reactivity to daily stressors. Journal of Personality, 59, 355-386. doi:10.1111/j.1467-6494.1991.tb00253.x

Bolger, N., \& Zuckerman, A. (1995). A framework for studying personality in the stress process. Journal of Personality and Social Psychology, 69, 890-902. doi:10.1037/0022-3514.69.5.890 
Bradley, J. R., \& Cartwright, S. (2002). Social support, job stress, health, and job satisfaction among nurses in the United Kingdom. International Journal of Stress Management, 9, 163-182. doi:10.1023/A:1015567731248

Breznitz, S., \& Goldberger, L. (1993). Stress research at a crossroads. In L. Goldberger \& S. Breznitz (Eds.), Handbook of stress: Theoretical and clinical aspects (2nd ed.). Cangelosi, J. D., Markham, F. S., \& Bounds, W. T. (1998). Factors related to nurse retention and turnover: An updated study. Health Marketing Quarterly, 15(3), 2543. doi:10.1300/J026v15n03_02

Carney, M. A., Armeli, S., Tennen, H., Affleck, G., \& O’Neil, T. P. (2000). Positive and negative daily events, perceived stress, and alcohol use: A diary study. Journal of Consulting and Clinical Psychology, 68, 788-798. doi:10.1037/0022006X.68.5.788

Carney, M. A., Tennen, H., Affleck, G., Del Boca, F. K., \& Kranzler, H. R. (1998). Levels and patterns of alcohol consumption using timeline follow-back, daily diaries and real-time "electronic interviews.” Journal of Studies on Alcohol, 59, $447-454$.

Carver, C. S., \& Scheier, M. F. (1994). Situational coping and coping dispositions in a stressful transaction. Journal of Personality and Social Psychology, 66, 184-195. doi:10.1037/0022-3514.66.1.184

Carver, C. S., Scheier, M. F., \& Weintraub, J. K. (1989). Assessing coping strategies: A theoretically based approach. Journal of Personality and Social Psychology, 56, 267-283. doi:10.1037/0022-3514.56.2.267

Cash, T. F., Novy, P. L., \& Grant, J. R. (1994). Why do women exercise? Factor analysis 
and further validation of the reasons for exercise inventory. Perceptual and Motor Skills, 78, 539-544. doi:10.2466/pms.1994.78.2.539

Centers for Disease Control and Prevention. (2011). Physical activity for everyone: The benefits of physical activity. Retrieved September 12, 2011, from http://www.cdc.gov/physicalactivity/everyone/health/index.html

Chandola, T., Brunner, E., \& Marmot, M. (2006). Chronic stress at work and the metabolic syndrome: prospective study. British Medical Journal, 332, 521-524. doi:10.1136/bmj.38693.435301.80

Chang, E. M., Hancock, K. M., Johnson, A., Daly, J., \& Jackson, D. (2005). Role stress in nurses: Review of related factors and strategies for moving forward. Nursing and Health Sciences, 7, 57-65. doi:10.1111/j.1442-2018.2005.00221.x

Charles, S. T., Piazza, J. R., Mogle, J., Sliwinski, M. J., \& Almeida, D. M. (2013). The wear and tear of daily stressors on mental health. Psychological Science, 24(5), 733-741. doi:10.1177/0956797612462222

Chipperfield, J. G. (1993). Incongruence between health perceptions and health problems: Implications for survival among seniors. Journal of Aging and Health, 5, 475-496. doi:10.1177/089826439300500404

Cockerham, W. C., Sharp, K., \& Wilcox, J. A. (1983). Aging and perceived health status. Journal of Gerontology, 38, 349-355. doi:10.1093/geronj/38.3.349

Cohen, L. H., Gunthert, K. C., Butler, A. C., O’Neill, S. C., \& Tolpin, L. H. (2005). Daily affective reactivity as a prospective predictor of depressive symptoms. Journal of Personality, 73, 1687-1714. doi:10.1111/j.0022-3506.2005.00363.x

Cohen, L. H., Gunthert, K. C., Butler, A. C., Parrish, B. P., Wenze, S. J., \& Beck, J. S. 
(2008). Negative affective spillover from daily events predicts early response to cognitive therapy for depression. Journal of Consulting and Clinical Psychology, 76, 955-965. doi:10.1037/a0014131

Cohen, S., \& Hamrick, N. (2003). Stable individual differences in physiological response to stressors: Implications for stress-elicited changes in immune related health. Brain, Behavior, and Immunity, 17, 407-414. doi:10.1016/S0889-1591(03)001107

Cohen, S., \& Hoberman, H. M. (1983). Positive events and social supports as buffers of life change stress. Journal of Applied Social Psychology, 13, 99-125. doi:10.1111/j.1559-1816.1983.tb02325.x

Cohen, S., Kessler, R. C., \& Gordon, L. U. (Eds.). (1997). Measuring stress: A guide for health and social scientists. Oxford University Press.

Colditz, G. A., Manson, J. E., \& Hankinson, S. E. (1997). The nurses' health study: 20year contribution to the understanding of health among women. Journal of Women's Health, 6, 49-62. doi:10.1089/jwh.1997.6.49

Conger, J. J. (1956). Reinforcement theory and the dynamics of alcoholism. Quarterly Journal of Studies on Alcohol, 17, 296-305.

Conn, V. S., Hafdahl, A. R., Cooper, P. S., Brown, L. M., \& Lusk, S. L. (2009). Metaanalysis of workplace physical activity interventions. American Journal of Preventive Medicine, 37(4), 330-339. doi:10.1016/j.amepre.2009.06.008

Cooper, M. L., Frone, M. R., Russell, M., \& Mudar, P. (1995). Drinking to regulate positive and negative emotions: A motivational model of alcohol use. Journal of Personality and Social Psychology, 69, 990-1005. doi:10.1037/0022- 
3514.69.5.990

Cooper, M. L., Russell, M., \& George, W. H. (1988). Coping, expectancies, and alcohol abuse: A test of social learning formulations. Journal of Abnormal Psychology, 97, 218-230. doi:10.1037/0021-843X.97.2.218

Cooper, M. L., Russell, M., Skinner, J. B., \& Windle, M. (1992). Development and validation of a three-dimensional measure of drinking motives. Psychological Assessment, 4, 123-132. doi:10.1037/1040-3590.4.2.123

Cox, W. M., \& Klinger, E. (1988). A motivational model of alcohol use. Journal of Abnormal Psychology, 97, 168-180. doi:10.1037/0021-843X.97.2.168

Crowne, D. P., \& Marlowe, D. (1960). A new scale of social desirability independent of psychopathology. Journal of Consulting Psychology, 24, 349-354.

Danna, K., \& Griffin, R. W. (1999). Health and well-being in the workplace: A review and synthesis of the literature. Journal of Management, 25, 357-384. doi:10.1177/014920639902500305

David, J. P., Green, P. J., Martin, R., \& Suls, J. (1997). Differential roles of neuroticism, extraversion, and event desirability for mood in daily life: An integrative model of top-down and bottom-up influences. Journal of Personality and Social Psychology, 73, 149-159. doi:10.1037/0022-3514.73.1.149

Dawson, D. A. (2003). Methodological issues in measuring alcohol use. Retrieved June 1, 2012, from http://pubs.niaaa.nih.gov/publications/arh27-1/18-29.htm Deese, N., Sears, L. E., Sinclair, R. R., Wright, R. R., Cadiz, D., Jacobs, L. M., ... Davidson, S. (2009). Bad vs. good: Do positive work experiences predict nurses' engagement? Presented at the Annual conference of the Society for Industrial and 
Organizational Psychology, New Orleans, LA.

DeLongis, A., Coyne, J. C., Dakof, G., Folkman, S., \& Lazarus, R. S. (1982).

Relationship of daily hassles, uplifts, and major life events to health status. Health Psychology, 1, 119-136. doi:10.1037/0278-6133.1.2.119

Derogatis, L. R., \& Coons, H. L. (1993). Self-report measures of stress. In L. Goldberger \& S. Breznitz (Eds.), Handbook of stress: Theoretical and clinical aspects (2nd ed.).

Derryberry, D., \& Rothbart, M. K. (1988). Arousal, affect, and attention as components of temperament. Journal of Personality and Social Psychology, 55, 958-966. doi:10.1037/0022-3514.55.6.958

Diener, E. (1984). Subjective well-being. Psychological Bulletin, 95, 542-575. doi:10.1037/0033-2909.95.3.542

Diener, E. (2000). Subjective well-being: The science of happiness and a proposal for a national index. American Psychologist, 55, 34-43. doi:10.1037/0003066X.55.1.34

Diener, E. (2006). Guidelines for national indicators of subjective well-being and illbeing. Applied Research in Quality of Life, 1, 151-157. doi:10.1007/s11482-0069007-X

Diener, E., Emmons, R. A., Larsen, R. J., \& Griffin, S. (1985). The satisfaction with life scale. Journal of Personality Assessment, 49, 71-75. doi:10.1207/s15327752jpa4901_13

Dishman, R. K., \& Jackson, E. M. (2000). Exercise, fitness, and stress. International Journal of Sport Psychology, 31(2), 175-203. 
Dohrenwend, B. P., Raphael, K. G., Schwartz, S., Stueve, A., \& Skodol, A. (1993). The structured event probe and narrative rating method for measuring stressful life events. In L. Goldberger \& S. Breznitz (Eds.), Handbook of stress: Theoretical and clinical aspects (2nd ed.).

Eckenrode, J. (1984). Impact of chronic and acute stressors on daily reports of mood. Journal of Personality and Social Psychology, 46, 907-918. doi:10.1037/00223514.46.4.907

Eckenrode, J., \& Bolger, N. (1997). Daily and within-day event measurement. In Measuring stress: A guide for health and social scientists (pp. 80-101). Oxford University Press.

Eisenberg, N., \& Fabes, R. A. (1992). Emotion, regulation, and the development of social competence. In Emotion and social behavior (Vol. 14, pp. 119-150).

Eisenberg, N., Fabes, R. A., Bernzweig, J., Karbon, M., Poulin, R., \& Hanish, L. (1993). The relations of emotionality and regulation to preschoolers' social skills and sociometric status. Child Development, 64, 1418-1438. doi:10.1111/j.14678624.1993.tb02961.x

Enders, C. K., \& Tofighi, D. (2007). Centering predictor variables in cross-sectional multilevel models: A new look at an old issue. Psychological Methods, 12, 121138. doi:10.1037/1082-989X.12.2.121

Epel, E. S., Lapidus, R., McEwen, B. S., \& Brownell, K. (2001). Stress may add bite to appetite in women: A laboratory study of stress-induced cortisol and eating behavior. Psychoneuroendocrinology, 26, 37-49. doi:10.1016/S03064530(00)00035-4 
Erdogan, B., Bauer, T. N., Truxillo, D. M., \& Mansfield, L. R. (2012). Whistle while you work: A review of the life satisfaction literature. Journal of Management, 38, 1038-1083. doi:10.1177/0149206311429379

Fabes, R. A., \& Eisenberg, N. (1997). Regulatory control and adults'stress-related responses to daily life events. Journal of Personality and Social Psychology, 73, 1107-1117. doi:10.1037/0022-3514.73.5.1107

Folkman, S., \& Lazarus, R. S. (1988). The relationship between coping and emotion: Implications for theory and research. Social Science \& Medicine, 26, 309-317. doi:10.1016/0277-9536(88)90395-4

Folkman, S., \& Moskowitz, J. T. (2000). Positive affect and the other side of coping. American Psychologist, 55, 647-654. doi:10.1037//0003-066X.55.6.647

Fredrickson, B. L. (1998). What good are positive emotions? Review of General Psychology, 2, 300-319. doi:10.1037/1089-2680.2.3.300

Fredrickson, B. L. (2001). The role of positive emotions in positive psychology: The broaden-and-build theory of positive emotions. American Psychologist, 56, 218226. doi:10.1037/0003-066X.56.3.218

Fredrickson, B. L., Mancuso, R. A., Branigan, C., \& Tugade, M. M. (2000). The undoing effect of positive emotions. Motivation and Emotion, 24, 237-258. doi:10.1023/A:1010796329158

Frone, M. R. (1999). Work stress and alcohol use. Alcohol Research \& Health, 23, 284 291.

Ganster, D. C., \& Schaubroeck, J. (1991). Work stress and employee health. Journal of Management, 17, 235-271. doi:10.1177/014920639101700202 
Gerberich, S. G., Church, T. R., McGovern, P. M., Hansen, H. E., Nachreiner, N. M., Geisser, M. S., ... Watt, G. D. (2004). An epidemiological study of the magnitude and consequences of work related violence: the Minnesota Nurses' Study. Occupational and Environmental Medicine, 61(6), 495-503. doi:10.1136/oem.2003.007294

Glass, D. C., McKnight, J. D., \& Valdimarsdottir, H. (1993). Depression, burnout, and perceptions of control in hospital nurses. Journal of Consulting and Clinical Psychology, 61, 147-155. doi:10.1037/0022-006X.61.1.147

Goldberg, L. R. (1999). A broad-bandwidth, public domain, personality inventory measuring the lower-level facets of several five-factor models. In I. Mervielde, I. Deary, F. De Fruyt, \& F. Ostendorf (Eds.), Personality psychology in Europe (Vol. 7, pp. 7-28). Tilburg, The Netherlands: Tilburg University Press.

Gomez, V., Krings, F., Bangerter, A., \& Grob, A. (2009). The influence of personality and life events on subjective well-being from a life span perspective. Journal of Research in Personality, 43, 345-354. doi:10.1016/j.jrp.2008.12.014

Gosling, S. D., Rentfrow, P. J., \& Swann, W. B. (2003). A very brief measure of the BigFive personality domains. Journal of Research in Personality, 37(6), 504-528. doi:10.1016/S0092-6566(03)00046-1

Greenglass, E. R., Burke, R. J., \& Moore, K. A. (2003). Reactions to increased workload: Effects on professional efficacy of nurses. Applied Psychology: An International Review, 52(4), 580-597. doi:10.1111/1464-0597.00152

Gunthert, K. C., Cohen, L. H., Butler, A. C., \& Beck, J. S. (2005). Predictive role of daily coping and affective reactivity in cognitive therapy outcome: Application of a 
daily process design to psychotherapy research. Behavior Therapy, 36, 77-88. doi:10.1016/S0005-7894(05)80056-5

Hancock, P. A., \& Warm, J. S. (1989). A dynamic model of stress and sustained attention. Human Factors, 31, 519-537. doi:10.1177/001872088903100503

Hartley, T. A., Violanti, J. M., Fekedulegn, D., Andrew, M. E., \& Burchfiel, C. M. (2007). Associations between major life events, traumatic incidents, and depression among Buffalo police officers. International Journal of Emergency Mental Health, 9(1), 25-35.

Häusser, J. A., Mojzisch, A., Niesel, M., \& Schulz-Hardt, S. (2010). Ten years on: A review of recent research on the job demand-control (-support) model and psychological well-being. Work \& Stress, 24, 1-35.

doi:10.1080/02678371003683747

Heeb, J.-L., \& Gmel, G. (2005). Measuring alcohol consumption: A comparison of graduated frequency, quantity frequency, and weekly recall diary methods in a general population survey. Addictive Behaviors, 30, 403-413. doi:10.1016/j.addbeh.2004.04.022

Hobfoll, S. E. (1989). Conservation of resources: A new attempt at conceptualizing stress. American Psychologist, 44, 513-524. doi:10.1037/0003-066X.44.3.513

Hobfoll, S. E. (2001). The influence of culture, community, and the nested-self in the stress process: Advancing conservation of resources theory. Applied Psychology, 50, 337-421. doi:10.1111/1464-0597.00062

Hockey, R. (1984). Varieties of attentional state: The effects of environment. In Varieties of attention (pp. 449-483). Academic Press, Inc. 
Hofer, S. M., \& Hoffman, L. (2007). Statistical analysis with incomplete data: A developmental perspective. In T. D. Little, J. A. Bovaird, \& N. A. Card (Eds.), Modeling contextual effects in longitudinal studies. (pp. 13-32). Mahwah, NJ, US: Lawrence Erlbaum Associates Publishers. Retrieved from http://psycnet.apa.org/psycinfo/2007-07587-002

Holmes, T. H., \& Rahe, R. H. (1967). The social readjustment rating scale. Journal of Psychosomatic Research, 11(2), 213-218. doi:10.1016/0022-3999(67)90010-4

Hox, J. J. (2010). Multilevel analysis: Techniques and applications (2nd ed.). New York, NY: Routledge.

Jamal, M., \& Baba, V. V. (1992). Shiftwork and department-type related to job stress, work attitudes and behavioral intentions: A study of nurses. Journal of Organizational Behavior, 13, 449-464. doi:10.1002/job.4030130503

Jex, S. M., \& Beehr, T. A. (1991). Emerging theoretical and methodological issues in the study of work-related stress. In Research in personnel and human resources management (Vol. 9, pp. 311-365). JAI Press Inc.

Johnson, J. V. (1996). Conceptual and methodological developments in occupational stress research: An introduction to state-of-the-art reviews I. Journal of Occupational Health Psychology, 1, 6-8. doi:10.1037/1076-8998.1.1.6

Judge, T. A., Erez, A., Bono, J. E., \& Thoresen, C. J. (2003). The core self-evaluations scale: Development of a measure. Personnel Psychology, 56(2), 303-331. doi:10.1111/j.1744-6570.2003.tb00152.x

Kanner, A. D., Coyne, J. C., Schaefer, C., \& Lazarus, R. S. (1981). Comparison of two modes of stress measurement: Daily hassles and uplifts versus major life events. 
Journal of Behavioral Medicine, 4, 1-39. doi:10.1007/BF00844845

Karasek, R. (2008). Low social control and physiological deregulation- - the stressdisequilibrium theory, towards a new demand-control model. Scandinavian Journal of Work, Environment \& Health Supplements, (6), 117-135.

Kendler, K. S., Kuhn, J., \& Prescott, C. A. (2004). The interrelationship of neuroticism, sex, and stressful life events in the prediction of episodes of major depression. American Journal of Psychiatry, 161, 631-636. doi:10.1176/appi.ajp.161.4.631

Keyes, C. L. M., Shmotkin, D., \& Ryff, C. D. (2002). Optimizing well-being: The empirical encounter of two traditions. Journal of Personality and Social Psychology, 82, 1007-1022. doi:10.1037//0022-3514.82.6.1007

Kivimäki, M., Virtanen, M., Elovainio, M., Kouvonen, A., Väänänen, A., \& Vahtera, J. (2006). Work stress in the etiology of coronary heart disease - a meta-analysis. Scandinavian Journal of Work, Environment \& Health, 32, 431-442. doi:10.5271/sjweh.1049

Koerner, S. S., \& Kenyon, D. B. (2007). Understanding “good days" and "bad days": Emotional and physical reactivity among caregivers for elder relatives. Family Relations, 56, 1-11. doi:10.1111/j.1741-3729.2007.00435.x

Kopp, M. S., Stauder, A., Purebl, G., Janszky, I., \& Skrabski, Á. (2008). Work stress and mental health in a changing society. European Journal of Public Health, 18, 238244. doi:10.1093/eurpub/ckm077

Kreuter, F., Presser, S., \& Tourangeau, R. (2009). Social desirability bias in CATI, IVR, and web surveys: The effects of mode and question sensitivity. Public Opinion Quarterly, 72, 847-865. doi:10.1093/poq/nfn063 
Lambert, V. A., \& Lambert, C. E. (2001). Literature review of role stress/strain on nurses: An international perspective. Nursing and Health Sciences, 3, 161-172. doi:10.1046/j.1442-2018.2001.00086.x

Lambert, V. A., Lambert, C. E., \& Yamase, H. (2003). Psychological hardiness, workplace stress and related stress reduction strategies. Nursing and Health Sciences, 5, 181-184. doi:10.1046/j.1442-2018.2003.00150.x

Lazarus, R. S. (1993a). Why we should think of stress as a subset of emotion. In L. Goldberger \& S. Breznitz (Eds.), Handbook of stress: Theoretical and clinical aspects (2nd ed.).

Lazarus, R. S. (1993b). From psychological stress to the emotions: a history of changing outlooks. Annual Review of Psychology, 44, 1-21. doi:10.1146/annurev.ps.44.020193.000245

Lazarus, R. S. (1999). Stress and emotion: A new synthesis. New York: Springer Publishing Company.

Lazarus, R. S., \& Folkman, S. (1984). Stress, appraisal, and coping. New York: Springer Publishing Company.

Lee, I.-M., Shiroma, E. J., Lobelo, F., Puska, P., Blair, S. N., \& Katzmarzyk, P. T. (2012). Effect of physical inactivity on major non-communicable diseases worldwide: an analysis of burden of disease and life expectancy. The Lancet, 380, 219-229. doi:10.1016/S0140-6736(12)61031-9

Lemke, S., Brennan, P. L., Schutte, K. K., \& Moos, R. H. (2007). Upward pressures on drinking: Exposure and reactivity in adulthood. Journal of Studies on Alcohol and Drugs, 68, 437-445. 
Lepore, S. J., Miles, H. J., \& Levy, J. S. (1997). Relation of chronic and episodic stressors to psychological distress, reactivity, and health problems. International Journal of Behavioral Medicine, 4, 39-59. doi:10.1207/s15327558ijbm0401_3

Lewinsohn, P. M., \& Graf, M. (1973). Pleasant activities and depression. Journal of Consulting and Clinical Psychology, 41, 261-268. doi:10.1037/h0035142

Liebman, B. (2009, October). What should I eat? Nutrition Action Health Letter. Retrieved September 11, 2011, from http://www.cspinet.org/nah/articles/whatshouldeat.html

Little, R. J. A., \& Rubin, D. B. (2002). Statistical analysis with missing data. Hoboken, N.J.: Wiley.

Liu, S., Wang, M., Zhan, Y., \& Shi, J. (2009). Daily work stress and alcohol use: Testing the cross-level moderation effects of neuroticism and job involvement. Personnel Psychology, 62, 575-597. doi:10.1111/j.1744-6570.2009.01149.x

Lucas, R. E. (2008). Personality and subjective well-being. In M. Eid \& R. J. Larsen (Eds.), The science of subjective well-being (pp. 171-194). New York: Guilford Press.

Lyubomirsky, S., King, L., \& Diener, E. (2005). The benefits of frequent positive affect: Does happiness lead to success? Psychological Bulletin, 131, 803-855. doi:10.1037/0033-2909.131.6.803

Macht, M., \& Simons, G. (2000). Emotions and eating in everyday life. Appetite, 35, 6571. doi:10.1006/appe.2000.0325

Macik-Frey, M., Quick, J. C., \& Nelson, D. L. (2007). Advances in occupational health: From a stressful beginning to a positive future. Journal of Management, 33, 809- 
840. doi:10.1177/0149206307307634

Mallers, M. H., Almeida, D. M., \& Neupert, S. D. (2005). Women's daily physical health symptoms and stressful experiences across adulthood. Psychology and Health, 20, 389-403. doi:10.1080/08870440512331317698

Mandler, G. (1993). Thought, memory, and learning: Effects of emotional stress. In L. Goldberger \& S. Breznitz (Eds.), Handbook of stress: Theoretical and clinical aspects (2nd ed.).

McEwen, B. S. (1998a). Protective and damaging effects of stress mediators. New England Journal of Medicine, 338, 171-179. doi:10.1056/NEJM199801153380307

McEwen, B. S. (1998b). Stress, adaptation, and disease: Allostasis and allostatic load. Annals of the New York Academy of Sciences, 840, 33-44. doi:10.1111/j.17496632.1998.tb09546.x

McEwen, B. S., \& Mendelson, S. (1993). Effects of stress on the neurochemistry and morphology of the brain: Counterregulation versus damage. In L. Goldberger \& S. Breznitz (Eds.), Handbook of stress: Theoretical and clinical aspects (2nd ed.). McGrath, A., Reid, N., \& Boore, J. (1989). Occupational stress in nursing. International Journal of Nursing Studies, 26, 343-358. doi:10.1016/0020-7489(89)90020-5

McLeroy, K. R., Bibeau, D., Steckler, A., \& Glanz, K. (1988). An ecological perspective on health promotion programs. Health Education \& Behavior, 15(4), 351-377. doi:10.1177/109019818801500401

McNeely, E. (2005). The consequences of job stress for nurses' health: Time for a checkup. Nursing Outlook, 53, 291-299. doi:10.1016/j.outlook.2005.10.001 
Ming, E. E., Adler, G. K., Kessler, R. C., Fogg, L. F., Matthews, K. A., Herd, J. A., \& Rose, R. M. (2004). Cardiovascular reactivity to work stress predicts subsequent onset of hypertension: The air traffic controller health change study. Psychosomatic Medicine, 66, 459-465. doi:10.1097/01.psy.0000132872.71870.6d Mischel, W., \& Shoda, Y. (1995). A cognitive-affective system theory of personality: Reconceptualizing situations, dispositions, dynamics, and invariance in personality structure. Psychological Review, 102, 246-268. doi:10.1037/0033295X.102.2.246

Mohr, C. D., Armeli, S., Tennen, H., Carney, M. A., Affleck, G., \& Hromi, A. (2001). Daily interpersonal experiences, context, and alcohol consumption: Crying in your beer and toasting good times. Journal of Personality and Social Psychology, 80, 489-500. doi:10.1037/0022-3514.80.3.489

Mohr, C. D., Armeli, S., Tennen, H., \& Todd, M. (2010). The complexities of modeling mood-drinking relationships: Lessons learned from daily process research. In J. D. Kassel (Ed.), Substance abuse and emotion. (pp. 189-216). Washington: American Psychological Association. Retrieved from http://content.apa.org/books/12067-008

Mohr, C. D., Brannan, D., Mohr, J., Armeli, S., \& Tennen, H. (2008). Evidence for positive mood buffering among college student drinkers. Personality and Social Psychology Bulletin, 34, 1249-1259. doi:10.1177/0146167208319385

Mohr, C. D., Brannan, D., Wendt, S., Jacobs, L. M., \& Wright, R. R. (2010). Daily mood-drinking slopes predicting alcohol outcomes among U.S. heavy drinkers. Presented at the Annual Meeting of the Kettil-Bruun Society, Lausanne, 
Switzerland.

Mohr, C. D., Brannan, D., Wendt, S., Jacobs, L. M., Wright, R. R., \& Wang, M. (2013). Daily mood-drinking slopes as predictors: A new take on drinking motives and related outcomes. Psychology of Addictive Behaviors, (Advance online publication). doi:10.1037/a0032633

Moos, R. H., \& Schaefer, J. A. (1993). Coping resources and processes: Current concepts and measures. In L. Goldberger \& S. Breznitz (Eds.), Handbook of stress: Theoretical and clinical aspects (2nd ed.).

Mroczek, D. K., \& Almeida, D. M. (2004). The effect of daily stress, personality, and age on daily negative affect. Journal of Personality, 72, 355-378. doi:10.1111/j.00223506.2004.00265.x

Muraven, M., \& Baumeister, R. F. (2000). Self-regulation and depletion of limited resources: Does self-control resemble a muscle? Psychological Bulletin, 126, 247-259. doi:10.1037//0033-2909.126.2.247

Muraven, M., Tice, D. M., \& Baumeister, R. F. (1998). Self-control as a limited resource: Regulatory depletion patterns. Journal of Personality and Social Psychology, 74, 774-789. doi:10.1037/0022-3514.74.3.774

Nave, C. S., Sherman, R. A., \& Funder, D. C. (2008). Beyond self-report in the study of hedonic and eudaimonic well- being: Correlations with acquaintance reports, clinician judgments and directly observed social behavior. Journal of Research in Personality, 42, 643-659. doi:10.1016/j.jrp.2007.09.001

Nesselroade, J. R. (2004). Intraindividual variability and short-term change. Gerontology, 50, 44-47. doi:10.1159/000074389 
Nesselroade, J. R., \& Salthouse, T. A. (2004). Methodological and theoretical implications of intraindividual variability in perceptual-motor performance. Journal of Gerontology: Psychological Sciences, 59, 49-55. doi:10.1093/geronb/59.2.P49

Neupert, S. D., Almeida, D. M., \& Charles, S. T. (2007). Age differences in reactivity to daily stressors: The role of personal control. Journal of Gerontology: Psychological Sciences, 62, 216-225.

Newman, E., O’Connor, D. B., \& Conner, M. (2007). Daily hassles and eating behaviour: The role of cortisol reactivity status. Psychoneuroendocrinology, 32, 125-132. doi:10.1016/j.psyneuen.2006.11.006

Nezlek, J. B., \& Gable, S. L. (2001). Depression as a moderator of relationships between positive daily events and day-to-day psychological adjustment. Personality and Social Psychology Bulletin, 27, 1692-1704. doi:10.1177/01461672012712012

O’Connor, D. B., \& O’Connor, R. C. (2004). Perceived changes in food intake in response to stress: The role of conscientiousness. Stress and Health, 20, 279-291. doi:10.1002/smi.1028

O’Connor, D. B., O’Connor, R. C., White, B. L., \& Bundred, P. E. (2000). Job strain and ambulatory blood pressure in British general practitioners: A preliminary study. Psychology, Health \& Medicine, 5, 241-250. doi:10.1080/713690191

Parrish, B. P., Cohen, L. H., \& Laurenceau, J.-P. (2011). Prospective relationship between negative affective reactivity to daily stress and depressive symptoms. Journal of Social and Clinical Psychology, 30, 270-296. doi:10.1521/jscp.2011.30.3.270 
Pavot, W., \& Diener, E. (1993). Review of the satisfaction with life scale. Psychological Assessment, 5, 164-172. doi:10.1037/1040-3590.5.2.164

Payne, N., Jones, F., \& Harris, P. R. (2010). A daily diary investigation of the impact of work stress on exercise intention realisation: Can planning overcome the disruptive influence of work? Psychology \& Health, 25, 111-129. doi:10.1080/08870440903337622

Peeters, F., Nicolson, N. A., Berkhof, J., Delespaul, P., \& deVries, M. (2003). Effects of daily events on mood states in major depressive disorder. Journal of Abnormal Psychology, 112, 203-211. doi:10.1037/0021-843X.112.2.203

Pennebaker, J. W. (1989). Confession, inhibition, and disease. In Advances in experimental social psychology (Vol. 22, pp. 211-244). Elsevier. Retrieved from http://linkinghub.elsevier.com/retrieve/pii/S0065260108603093

Petruzzello, S. J., Landers, D. M., Hatfield, B. D., Kubitz, K. A., \& Salazar, W. (1991). A meta-analysis on the anxiety-reducing effects of acute and chronic exercise. Sports Medicine, 11(3), 143-182. doi:10.2165/00007256-199111030-00002

Piazza, J. R., Charles, S. T., Sliwinski, M. J., Mogle, J., \& Almeida, D. M. (2012). Affective reactivity to daily stressors and long-term risk of reporting a chronic physical health condition. Annals of Behavioral Medicine, 45(1), 110-120. doi:10.1007/s12160-012-9423-0

Pillow, D. R., Zautra, A. J., \& Sandler, I. (1996). Major life events and minor stressors: Identifying mediational links in the stress process. Journal of Personality and Social Psychology, 70, 381-394. doi:10.1037/0022-3514.70.2.381

Pynoos, R. S., Sorenson, S. B., \& Steinberg, A. M. (1993). Interpersonal violence and 
traumatic stress reactions. In L. Goldberger \& S. Breznitz (Eds.), Handbook of stress: Theoretical and clinical aspects (2nd ed.).

Quick, J. C. (1999). Occupational health psychology: The convergence of health and clinical psychology with public health and preventive medicine in an organizational context. Professional Psychology: Research and Practice, 30, 123-128. doi:10.1037/0735-7028.30.2.123

Quick, J. D., Horn, R. S., \& Quick, J. C. (1987). Health consequences of stress. Journal of Organizational Behavior Management, 8(2), 19-36. doi:10.1300/J075v08n02_03

Rabkin, J. G. (1993). Stress and psychiatric disorders. In L. Goldberger \& S. Breznitz (Eds.), Handbook of stress: Theoretical and clinical aspects (2nd ed.).

Radloff, L. S. (1977). The CES-D scale: A self-report depression scale for research in the general population. Applied Psychological Measurement, 1, 385-401. doi:10.1177/014662167700100306

Raudenbush, S. W., Bryk, A. S., Cheong, Y. F., Congdon, R. T., \& du Toit, M. (2011). HLM 7 for Windows. Lincolnwood, IL: Scientific Software International, Inc.

Repetti, R. L. (1993). Short-term effects of occupational stressors on daily mood and health complaints. Health Psychology, 12, 125-131. doi:10.1037/02786133.12 .2 .125

Rose, G., Kumlin, L., Dimberg, L., Bengtsson, C., Orth-Gomer, K., \& Cai, X. (2006). Work-related life events, psychological well-being and cardiovascular risk factors in male Swedish automotive workers. Occupational Medicine, 56, 386-392. doi:10.1093/occmed/kq1037 
Russell, D. W., \& Cutrona, C. E. (1991). Social support, stress, and depressive symptoms among the elderly: Test of a process model. Psychology and Aging, 6, 190-201. doi:10.1037/0882-7974.6.2.190

Salovey, P., Hsee, C. K., \& Mayer, J. D. (1993). Emotional intelligence and the selfregulation of affect. In D. M. Wegner \& J. W. Pennebaker (Eds.), Handbook of mental control (pp. 258-277). Prentice-Hall, Inc.

Salovey, P., Rothman, A. J., Detweiler, J. B., \& Steward, W. T. (2000). Emotional states and physical health. American Psychologist, 55, 110-121. doi:10.1037/0003066X.55.1.110

Santor, D. A., \& Coyne, J. C. (1997). Shortening the CES-D to improve its ability to detect cases of depression. Psychological Assessment, 9, 233-243. doi:10.1037/1040-3590.9.3.233

Schaufeli, W. B., \& Bakker, A. B. (2004). Job demands, job resources, and their relationship with burnout and engagement: A multi-sample study. Journal of Organizational Behavior, 25, 293-315. doi:10.1002/job.248

Schnall, P. L., Landsbergis, P. A., \& Baker, D. (1994). Job strain and cardiovascular disease. Annual Review of Public Health, 15, 381-411. doi:10.1146/annurev.pu.15.050194.002121

Schwebel, D. C., \& Suls, J. (1999). Cardiovascular reactivity and neuroticism: Results from a laboratory and controlled ambulatory stress protocol. Journal of Personality, 67, 67-92. doi:10.1111/1467-6494.00048

Selye, H. (1993). History of the stress concept. In L. Goldberger \& S. Breznitz (Eds.), Handbook of stress: Theoretical and clinical aspects (2nd ed.). 
Serido, J., Almeida, D. M., \& Wethington, E. (2004). Chronic stressors and daily hassles: Unique and interactive relationships with psychological distress. Journal of Health and Social Behavior, 45, 17-33. doi:10.1177/002214650404500102

Sherbourne, C. D., Edelen, M. O., Zhou, A., Bird, C., Duan, N., \& Wells, K. B. (2008). How a therapy-based quality improvement intervention for depression affected life events and psychological well-being over time: A 9-year longitudinal analysis. Medical Care, 46, 78-84. doi:10.1097/MLR.0b013e318148478d

Shirom, A., Westman, M., Shamai, O., \& Carel, R. S. (1997). Effects of work overload and burnout on cholesterol and triglycerides levels: The moderating efffects of emotional reactivity among male and female employees. Journal of Occupational Health Psychology, 2, 275-288. doi:10.1037/1076-8998.2.4.275

Shmueli, A. (1999). Subjective health status and health values in the general population. Medical Decision Making, 19, 122-127.

Shoda, Y., Mischel, W., \& Wright, J. C. (1994). Intraindividual stability in the organization and patterning of behavior: Incorporating psychological situations into the idiographic analysis of personality. Journal of Personality and Social Psychology, 67, 674-687. doi:10.1037/0022-3514.67.4.674

Sinclair, R. R., Mohr, C. D., Davidson, S., Sears, L. E., Deese, N., Wright, R. R., ... Cadiz, D. (2009). The Oregon Nurse Retention Project: Final report to the Northwest Health Foundation (Unpublished technical report) (p. 82).

Sliwinski, M. J., Almeida, D. M., Smyth, J., \& Stawski, R. S. (2009). Intraindividual change and variability in daily stress processes: Findings from two measurementburst diary studies. Psychology and Aging, 24, 828-840. doi:10.1037/a0017925 
Snijders, T., \& Bosker, R. (1999). Multilevel analysis: An introduction to basic and advanced multilevel modeling. Sage Publications.

Sparks, S. A., Corcoran, K. J., Nabors, L. A., \& Hovanitz, C. A. (2005). Job satisfaction and subjective well-being in a sample of nurses. Journal of Applied Social Psychology, 35, 922-938. doi:10.1111/j.1559-1816.2005.tb02153.x

Spector, P. E., Chen, P. Y., \& O’Connell, B. J. (2000). A longitudinal study of relations between job stressors and job strains while controlling for prior negative affectivity and strains. Journal of Applied Psychology, 85, 211-218. doi:10.1037//0021-9010.85.2.211

Steger, M. F., \& Kashdan, T. B. (2009). Depression and everyday social activity, belonging, and well-being. Journal of Counseling Psychology, 56, 289-300. doi:10.1037/a0015416

Stein, M., \& Miller, A. H. (1993). Stress, the immune system, and health and illness. In L. Goldberger \& S. Breznitz (Eds.), Handbook of stress: Theoretical and clinical aspects (2nd ed.).

Stephens, C., \& Pugmire, L. A. (2008). Daily organisational hassles and uplifts as determinants of psychological and physical health in a sample of New Zealand police. International Journal of Police Science and Management, 10, 179-191. doi:10.1350/ijps.2008.10.2.73

Steptoe, A., Lipsey, Z., \& Wardle, J. (1998). Stress, hassles and variations in alcohol consumption, food choice and physical exercise: A diary study. British Journal of Health Psychology, 3, 51-63. doi:10.1111/j.2044-8287.1998.tb00555.x

Stetson, B. A., Rahn, J. M., Dubbert, P. M., Wilner, B. I., \& Mercury, M. G. (1997). 
Prospective evaluation of the effects of stress on exercise adherence in community-residing women. Health Psychology, 16(6), 515-520.

doi:10.1037/0278-6133.16.6.515

Stone, A. A., \& Neale, J. M. (1984). Effects of severe daily events on mood. Journal of Personality and Social Psychology, 46, 137-144. doi:10.1037/00223514.46 .1 .137

Suh, E., Diener, E., \& Fujita, F. (1996). Events and subjective well-being: Only recent events matter. Journal of Personality and Social Psychology, 70, 1091-1102. doi:10.1037/0022-3514.70.5.1091

Suls, J., \& Martin, R. (2005). The daily life of the garden-variety neurotic: Reactivity, stressor exposure, mood spillover, and maladaptive coping. Journal of Personality, 73, 1485-1510. doi:10.1111/j.1467-6494.2005.00356.x

Tabachnick, B. G., \& Fidell, L. S. (2007). Using multivariate statistics (5th ed.). New York, NY: Allyn \& Bacon.

Tessler, R., \& Mechanic, D. (1978). Psychological distress and perceived health status. Journal of Health and Social Behavior, 19, 254-262.

Thayer, R. E. (1987). Energy, tiredness, and tension effects of a sugar snack versus moderate exercise. Journal of Personality and Social Psychology, 52, 119-125. doi:10.1037/0022-3514.52.1.119

Thayer, R. E., Newman, J. R., \& McClain, T. M. (1994). Self-regulation of mood: Strategies for changing a bad mood, raising energy, and reducing tension. Journal of Personality and Social Psychology, 67, 910-925. doi:10.1037/00223514.67.5.910 
Totterdell, P., Wood, S., \& Wall, T. (2006). An intra-individual test of the demandscontrol model: A weekly diary study of psychological strain in portfolio workers. Journal of Occupational and Organizational Psychology, 79, 63-84. doi:10.1348/096317905X52616

Tugade, M. M., Fredrickson, B. L., \& Feldman Barrett, L. (2004). Psychological resilience and positive emotional granularity: Examining the benefits of positive emotions on coping and health. Journal of Personality, 72, 1161-1190. doi:10.1111/j.1467-6494.2004.00294.x

Turner, R. J., \& Wheaton, B. (1997). Checklist measurement of stressful life events. In S. Cohen, R. C. Kessler, \& L. U. Gordon (Eds.), Measuring Stress: A Guide for Health and Social Scientists. Oxford University Press.

Vingerhoets, A. J. J. M., \& Marcelissen, F. H. G. (1988). Stress research: Its present status and issues for future developments. Social Science \& Medicine, 26, 279291. doi:10.1016/0277-9536(88)90392-9

Wagner, B. M., Compas, B. E., \& Howell, D. C. (1988). Daily and major life events: A test of an integrative model of psychosocial stress. American Journal of Community Psychology, 16, 189-205. doi:10.1007/BF00912522

Walls, T. A., Jung, H., \& Schwartz, J. E. (2006). Multilevel models for intensive longitudinal data. In T. A. Walls \& J. L. Schafer (Eds.), Models for intensive longitudinal data (pp. 3-37). Oxford University Press.

Wang, M., Liu, S., Zhan, Y., \& Shi, J. (2010). Daily work-family conflict and alcohol use: Testing the cross-level moderation effects of peer drinking norms and social support. Journal of Applied Psychology, 95(2), 377-386. doi:10.1037/a0018138 
Watson, D., \& Pennebaker, J. W. (1989). Health complaints, stress, and distress: Exploring the central role of negative affectivity. Psychological Review, 96, 234254. doi:10.1037/0033-295X.96.2.234

Wegner, D. M., \& Pennebaker, J. W. (1993). Changing our minds: An introduction to mental control. In D. M. Wegner \& J. W. Pennebaker (Eds.), Handbook of mental control (pp. 1-12). Prentice-Hall, Inc.

Wichers, M., Geschwind, N., Jacobs, N., Kenis, G., Peeters, F., Derom, C., ... van Os, J. (2009). Transition from stress sensitivity to a depressive state: Longitudinal twin study. The British Journal of Psychiatry, 195, 498-503.

doi:10.1192/bjp.bp.108.056853

Wilsnack, R. W., Vogeltanz, N. D., Wilsnack, S. C., \& Harris, T. R. (2000). Gender differences in alcohol consumption and adverse drinking consequences: crosscultural patterns. Addiction, 95, 251-265. doi:10.1046/j.13600443.2000.95225112.x

Zapf, D., Dormann, C., \& Frese, M. (1996). Longitudinal studies in organizational stress research: A review of the literature with reference to methodological issues. Journal of Occupational Health Psychology, 1, 145-169. doi:10.1037/10768998.1.2.145

Zautra, A. J. (2003). Emotions, stress, and health. Oxford University Press. 
Appendix A

Measures by Timepoint

\begin{tabular}{lll}
\multicolumn{1}{c}{ Measure } & \multicolumn{1}{c}{ Timepoint } & \multicolumn{1}{c}{ Reference } \\
\hline Age & Signup & \\
Sex & Signup & \\
Neuroticism & Wave 1 & Goldberg, 1999, Judge et al., 2003 \\
Perceived Health & Wave 1 and Wave 2 & Chipperfield, 1993 \\
Negative Events & Weekly & Developed (Sinclair et al., 2009) \\
Health Behaviors & Weekly & Based on NIAAA quantity-frequency \\
& & questions (Armor et al., 1978) \\
SWLS & Wave 2 & Diener, et al., 1985 \\
CES-D & Wave 2 & Santor \& Coyne, 1997 \\
& & \\
& \multicolumn{2}{c}{ Questionnaire Items }
\end{tabular}

Neuroticism:

1. Sometimes I feel depressed.

2. I am capable of coping with most of my problems.

3. There are times when things look pretty bleak and hopeless to me.

4. I have frequent mood swings.

5. I am not easily bothered by things.

Perceived Health:

1. Compared to other people your age, how would you rate your health?

Weekly Negative Events:

1. I did not have enough time to finish my tasks during my scheduled shift.

2. The organization I work for expected more of me than my skills and/or education provide.

3. I had an interpersonal conflict with patients/families (e.g., I was ignored, treated unprofessionally, doubted, disrespected).

4. I had an interpersonal conflict with a physician (e.g., I was ignored, treated unprofessionally, doubted, disrespected).

5. I had an interpersonal conflict with a coworker (e.g., I was ignored, treated unprofessionally, doubted, disrespected).

6. I had an interpersonal conflict with a manager (e.g., I was ignored, treated unprofessionally, doubted, disrespected).

7. I was micromanaged.

8. I was sexually harassed.

9. I was discriminated against because of my race, sex, age, sexual orientation, or religious beliefs. 
10. I was asked to provide patient care that was against my personal and/or professional beliefs or values.

11. I felt helpless when a patient failed to improve.

12. I had a patient whose health unexpectedly declined.

13. I received incomplete or unclear information about a patient's condition.

14. I experienced problems with equipment or supplies (e.g., computer system problems, unfamiliar equipment, misplaced supplies).

15. I did not have enough RNs with the specific education or skills needed for this unit.

16. I did not have enough experienced RNs to take care of patient needs.

17. I did not have enough RNs to meet patient care demands.

18. I did not have enough staff to adequately cover the unit.

19. Scheduled staff were late or absent for a shift.

20. Requests for additional staff were denied.

21. Requested staff were approved but did not arrive in time.

Health Behavior:

1. I ate healthy foods

2. On how many days did you engage in aerobic or physical activity, such as walking, jogging, or cycling?

3. In the past 7 days, how many minutes did you engage in aerobic or physical activity on an average day?

4. In the past 7 days, on how many days did you drink alcohol?

5. On a typical day, how many alcoholic drinks did you have?

\section{CES-D:}

1. You were bothered by things that usually do not bother you.

2. You felt that you could not shake off the "blues" even with help from your family or friends. 1

3. You had trouble keeping your mind on what you were doing.

4. You felt depressed.

5. You felt that everything you did was an effort.

6. Your sleep was restless.

7. You were happy.

8. You enjoyed life.

9. You felt sad.

Satisfaction With Life Scale:

1. In most ways my life is close to my ideal.

2. The condition of my life is excellent.

3. I am satisfied with my life.

4. So far I have gotten the important things I want in life.

5. If I could live my life over, I would change almost nothing. 